\title{
Procurement when Price and Quality Matter*
}

\author{
John Asker ${ }^{\dagger}$ and Estelle Cantillon ${ }^{\ddagger}$
}

November 10, 2006

\begin{abstract}
A buyer seeks to procure a good characterized by its price and its quality from suppliers who have private information about their cost structure (fixed cost + marginal cost of providing quality). We solve for the optimal buying procedure, i.e. the procedure that maximizes the buyer's expected utility. We then use the optimal procedure as a theoretical and numerical benchmark to study practical and simple buying procedures such as scoring auctions and negotiation. Specifically, we derive the restrictions that these simpler procedures place on allocations and compare them with the optimal allocations to generate insights about the properties of these simpler procedures and identify environments where they are likely to do well. We also use the optimal procedure benchmark to compare the performance of these procedures numerically. We find that scoring auctions are able to extract a good proportion of the surplus from being a strategic buyer, that is, the difference between the expected revenue from the optimal mechanism and the efficient auction. Sequential procedures (to which many negotiation processes belong) do less well, and, in fact, often do worse than simply holding an efficient auction. In each case, we identify the underlying reason for these results.
\end{abstract}

Keywords: optimal auction, multi-attribute auction, differentiated product, multidimensional screening, scoring auction, negotiation, bargaining, procurement.

JEL Codes: D44, D82, C78, L24, L22

\footnotetext{
${ }^{*}$ We thank Mark Armstrong, Heski Bar-Isaac, Micael Castanheira, Mathias Dewatripont, Philippe Jehiel, Georg Kirchsteiger, Patrick Legros, Nicolas Sahuguet as well as audiences at the Barcelona workshop on auctions, CEMFI, Paris School of Economics GREQAM, IDEI Toulouse, LSE, UCL, NYU IO Day, and the Duke-Northwestern-Texas IO Theory conference for helpful comments, and Alexis Walckiers for his careful proof-reading and many expositional suggestions. Financial support from a FNRS research grant is gratefully acknowledged. This paper supersedes an earlier paper entitled "Optimal Procurement when both Price and Quality Matter."

${ }^{\dagger}$ Leonard N. Stern School of Business, NYU. Email: jasker@stern.nyu.edu

${ }^{\ddagger}$ FNRS, ECARES (Université Libre de Bruxelles) and CEPR. Email: Estelle.Cantillon@ulb.ac.be
} 


\section{Introduction}

Procurement rarely involves considerations solely based on price. Instead, concerns about the quality of the good or service provided are often important to the final decision. In this paper, we consider how a buyer who cares about quality should structure his purchasing process when suppliers compete for a single procurement contract and we evaluate the performance of simpler (but suboptimal) procedures.

When suppliers' private information about their costs can be captured by a one-dimensional parameter, the answer to the first question is well-known (Laffont and Tirole, 1987, and Che, 1993). In addition, Che (1993) provides a partial answer to the second question by showing that a scoring auction implements the optimal mechanism. This paper extends the analysis of the first question to environments with multidimensional private information and answers the second question more exhaustively and for several alternative procedures.

The two distinguishing features of our model are that suppliers' private information about their cost structure is multidimensional and that quality is contractable and endogenously determined as part of the procurement process. US State Highway Authorities' procurement for highway repair jobs illustrates these aspects of the contracting environment. ${ }^{1}$ For high density traffic areas, these agencies care about the cost of the job and the time in which the job will be completed. A contractor may be able to speed up the job by hiring extra labor, by using some equipment more intensively, or by shifting some resources from other jobs. Hence, suppliers' quality (here, the time they need to complete the job) is not fixed but is endogenous, with increased quality incurring a higher cost. Moreover, this marginal cost of quality is likely to vary across potential contractors in a way that is not observable to their competitors. Therefore, it represents one dimension of private information. However, there are other sources of unobserved cost heterogeneity. These include the contractors' material costs, existing contractual obligations and organizational structure, which combine to determine the fixed cost of undertaking a job at any quality level. Thus, private information is likely to be better captured by a multidimensional parameter.

We first derive the optimal procurement mechanism in a model where each potential supplier has private information about two components of her cost structure: her fixed cost and her marginal cost of providing quality. Costs on each dimension can be high or low, and we allow for any pattern of correlation between a supplier's fixed cost and her marginal cost. Across bidders, costs are independently distributed. The buyer's objective is to maximize his expected utility subject to the suppliers' participation and incentive compatibility constraints.

The optimal procurement mechanism differs significantly from its counterpart in one-dimensional environments. It depends finely on the exact parameters of the problem, including the number of suppliers. Moreover, it is not easily amenable to implementation by a simple-looking auction

\footnotetext{
${ }^{1}$ See for instance Arizona Department of Transport (2002) and Herbsman et al. (1995).
} 
format, unlike its one-dimensional counterpart. The source of these discrepancies can be traced back to the well-known endogeneity of the direction in which incentive compatibility constraints bind in multidimensional screening problems.

While the fragility of the intuitions gained from one-dimensional models is useful to illustrate, the research agenda on multidimensional screening has left the economist concerned with the application of mechanism design on unsure footing. In this paper, we take a new approach and use the characterization of the optimal mechanism as a benchmark to investigate the performance of practical and simpler buying procedures.

This benchmark role plays out at two levels. At the theoretical level, we can compare the allocation (probabilities of getting the contract and qualities delivered) of the optimal mechanism and that of any other mechanism of interest to understand its advantages and disadvantages.

At a numerical level, the characterization of the optimal mechanism solves what is essentially a free parameter problem in interpreting numerical comparisons of the performance of other procedures. Without such a characterization, the only readily available benchmark is the efficient mechanism. Unfortunately, the efficient mechanism is not useful on its own. To illustrate, suppose that, for some set of parameters, the efficient mechanism generates an expected utility for the buyer of 1 , while the mechanism of interest returns 2. This looks like a $100 \%$ improvement in revenue. However, by adding 9 to the buyer's utility function, we could well generate expected utilities of 10 and 11 respectively. Now the improvement looks like only $10 \%$. The characterization of the optimal mechanism gives an extra point of comparison. Suppose the optimal mechanism returns an expected utility of 3 (or 12). This allows us to conclude that the mechanism of interest captures $50 \%$ of the rents available from being a strategic buyer (that is, the difference between the revenue from the optimal mechanism and the efficient mechanism).

We apply this new approach to evaluate the performance of the efficient auction, scoring auctions and sequential procedures where suppliers are approached one at a time. Our motivation for looking at these procedures is twofold. First, Asker and Cantillon (2006) have shown that scoring auctions dominate price-only auctions, beauty contests and menu auctions. Thus, scoring auctions are an obvious candidate for a simple second-best procedure. Second, negotiation is often preferred by buyers when quality matters and our model of sequential procedures bounds many realistic models of negotiation.

We characterize the allocations that can be implemented by a scoring auction (Theorem 2) and derive the optimal sequential procedure (Theorem 3). By definition, both procedures underperform against the optimal buying mechanism. The comparison with the allocation generated by the optimal mechanism highlights several characteristics of these alternatives. First, scoring auctions can replicate the allocation probabilities of the optimal procedure in many cases. Where scoring auctions fall short of the optimal auction concerns their inflexibility in terms of qualities. Second, the efficient auction can be implemented by a scoring auction. Thus, scoring auctions can always do 
weakly better than the efficient procedure. Third, sequential mechanisms are inherently inefficient and can never replicate the allocation probabilities of the optimal procedure. However, we identify two classes of environments where they can do better than the efficient auction thanks to the distortion in production or in allocation probabilities that they generate.

We further investigate these questions numerically by evaluating the proportion of the surplus to being strategic that simpler procedures capture across a wide range of environments. To do this, we compute an upper bound to the expected utility from these procedures by deriving the optimal scoring auction and the optimal sequential procedure. We find that the optimal scoring auction does very well and on average captures more than two thirds of the surplus, given the parameters we consider. Because this result holds for the upper bound and may not extend for all scoring auctions, we also investigate the performance of scoring auctions that use "naïve" scoring rules that distort the buyer's true preferences in the same direction as the optimal scoring auction but in an arbitrary way. We find that small deviations from the buyer's true preferences often uniformly increase his expected utility. We then turn to sequential procedures. We find that the optimal sequential procedure does very badly and often worse than the efficient auction, except when the fixed and marginal costs are highly correlated, or when there is little uncertainty about suppliers' fixed costs. Because these two classes of environments are near one-dimensional environments, it seems safe to claim that efficient auctions generally dominate sequential procedures when private information is multidimensional.

Related literature. This paper is related to the literatures on procurement and on multidimensional screening.

The literature on procurement is organized around several themes, including the question of how to take factors other than price into account in the procurement process (Laffont and Tirole, 1987, Che, 1993, Branco, 1997, Ganuza and Pechlivanos, 2000, Rezende, 2003, de Frutos and Pechlivanos, 2004), the impact of the potential non-contractability of quality (Klein and Leffler, 1981; Taylor, 1993; Manelli and Vincent, 1995; Morand and Thomas, 2002; Che and Gale, 2003), and the impact of moral hazard and renegotiation (Bajari and Tadelis, 2001, Bajari, McMillan and Tadelis, 2004). See Che (2006) for an overview.

Our paper fits squarely into the first group and we abstract from the other issues. Our contribution to this literature is twofold. First, we extend prior analyses of optimal procurement to the richer environment where private information is multidimensional. Laffont and Tirole (1987) and Che (1993) characterize the optimal buying mechanism when private information is one-dimensional (the marginal cost of providing quality). Under some regularity conditions, the optimal buying scheme distorts the quality provided by the suppliers downwards relative to their first best levels. The optimal level of distortion is independent of the number of suppliers, a property known as the "separation between screening and selection" (Laffont and Tirole, 1987). In addition, except for the presence of a reserve price, the contract is always allocated efficiently. Finally, Che shows 
that a scoring auction with a scoring rule that is linear in price implements the optimal scheme. Our analysis shows that these results depend heavily on the assumption of one-dimensional signals: except for the downward distortion of quality, none of these properties holds when we move to a multidimensional setting. Second, we evaluate existing buying procedures against the benchmark of the optimal scheme. Other papers compare the performance of different procedures: Dasgupta and Spulber (1989), Che (1993) and Chen-Ritzo et al. (2003) compare the scoring auction, which turns out to be optimal in their setting, with price-only auctions, Asker and Cantillon (2006) compare the scoring auction with price-only auctions, beauty contests, and menu auctions, Manelli and Vincent (1995) and Bulow and Klemperer (1996) compare (two different models of) negotiation with auctions. Except for Asker and Cantillon (2006), all these papers are restricted to one-dimensional private information. Moreover, our paper goes beyond simply ranking procedures by providing a quantitative assessment of the difference in expected utility, and identifying environments where these alternative procedures are likely to perform well.

This paper also contributes to the literature in multidimensional screening. Rochet and Stole (2003) present a recent survey of the contracting applications of multidimensional screening. Auction applications include the optimal multi-unit auction problems studied by Armstrong (2000), Avery and Henderschott (2000), Manelli and Vincent (2004) and Malakhov and Vohra (2004), and the optimal auction with externalities studied by Jehiel et al. (1999). Unlike contracting environments, our problem involves a resource constraint because the contract can only be allocated to one supplier. Unlike multi-unit auction environments, quality in our problem introduces some non-linearity. Hence, none of the existing characterization results applies to our problem and the method we use to solve for the solution is somewhat different from the methods used in these papers (even if the underlying principle is the same).$^{2}$

Through our emphasis on second-best mechanisms, our work echoes the research agenda laid out in Wilson (1993) of identifying simple and robust second best mechanisms. Our contribution here is in leveraging the characterization of the optimal mechanism to analyze second-best candidates in auction environments with multidimensional private information.

\section{Model}

We consider a buyer who wants to buy an indivisible good for which there are $N$ potential suppliers. The good is characterized by its price, $p$, and its quality, $q$.

Preferences. The buyer values the good $(p, q)$ at $v(q)-p$, where $v_{q}>0$ (we assume that $v_{q}(0)=\infty$ and $\lim _{q \rightarrow \infty} v_{q}(q)=0$ to ensure an interior solution) and $v_{q q}<0$. Supplier $i$ 's profit from selling $\operatorname{good}(p, q)$ is given by $p-\theta_{1}^{i}-\theta_{2}^{i} q$, where $\theta_{1}^{i} \in\{l, h\}$ and $\theta_{2}^{i} \in\{L, H\}(l<h$ and $0<L<H$.). For

\footnotetext{
${ }^{2}$ Asker and Cantillon (2005, section 6) investigate in more detail the formal analogies and differences with existing multidimensional screening problems.
} 
future reference, let $\Delta \theta_{1}=h-l$ and $\Delta \theta_{2}=H-L$. Given the binomial support of $\theta_{1}$ and $\theta_{2}$, there are four supplier types: $(h, H),(l, H),(h, L),(l, L)$, which we denote for brevity $h H, l H, h L$ and $l L$. We will sometimes use $\left(\theta_{1 k}, \theta_{2 k}\right)$ to denote supplier type $k$. For example, $\left(\theta_{1 l H}, \theta_{2 l H}\right)=(l, H)$. Note that the buyer and the suppliers are risk neutral.

Social welfare. Let $W_{k}(q)=v(q)-\theta_{1 k}-\theta_{2 k} q$, the social welfare associated with giving the contract to type $k$ with quality $q$. Define $W_{k}^{F B}=\max _{q} W_{k}(q)$. Given the single crossing condition, $q_{l H}^{F B}=q_{h H}^{F B}<q_{h L}^{F B}=q_{l L}^{F B}$ (to save on notation we will use the short-hand notation $\bar{q}$ and $\underline{q}$ to describe the first best levels of qualities, $\bar{q}<\underline{q}$ ).

Our assumptions thus far yield an incomplete ordering of types in terms of the first best levels of welfare they generate. To simplify the analysis, we restrict attention to the case where $W_{l H}^{F B}<$ $W_{h L}^{F B}$. This assumption does not affect the method we use or the results we get (in particular, Theorems 2 and 3 do not need this assumption). It simply reduces the number of cases we need to consider when we characterize the optimal mechanism (Theorem 1). Under this assumption, having a low marginal cost for delivering a higher quality product is more important than having a low fixed cost, at least in the first best solution. This case includes, as a limit, the case where firms only differ in their marginal cost parameter, which has been studied by Laffont and Tirole (1987), Che (1993) and Branco (1997). The natural ordering of types is thus $l L \succ h L \succ l H \succ h H$. Note that this assumption implies that $\Delta \theta_{1}-\Delta \theta_{2} \underline{q}<0$.

Information. Preferences are common knowledge among suppliers and the buyer, with the exception of suppliers' types, $\left(\theta_{1 i}, \theta_{2 i}\right), i=1, \ldots, N$, which are privately observed by each supplier. Types are independently and symmetrically distributed across suppliers, in the sense that the probability of supplier $i$ being of some type is independent of other suppliers' types, but the ex-ante distribution of types is the same for all bidders. Thus we can write the probability of each type as $\alpha_{k}>0$, $k \in\{h H, l H, h L, l L\}$. Notice that we do not put any restriction on the $\alpha_{k}$ 's except for the fact that they need to sum to one. Any pattern of correlation among a supplier's fixed cost and her marginal cost is allowed.

Note: The 2-by-2 discrete type space considered here is a concession to the practical difficulties of optimal screening problems in multidimensional environments. Armstrong (1996) and Rochet and Choné (1998) consider a nonlinear pricing problem in a continuous type-space. Their analyses suggest that for tractability a lot of ex ante structure needs to be imposed at the expense of economic richness. Much of the recent work in multidimensional screening has tended to focus on discrete type spaces (Armstrong, 2000; Avery and Henderschott, 2000; Malakhov and Vohra, 2004). In the conclusions, we discuss the applicability of our results to richer informational environments. 


\section{Characterization of the Optimal Mechanism}

The buyer's problem is to find a mechanism that maximizes his expected utility from the procurement process. For simplicity, we assume that the buyer buys with probability one (that is, we assume non exclusion). ${ }^{3}$ A direct revelation mechanism in this setting is a mapping from the announcements of all suppliers, $\left\{\theta_{1 i}, \theta_{2 i}\right\}_{i=1}^{N}$, into probabilities of getting the contract, the level of quality to deliver and a money transfer.

Given that the buyer's preference over quality levels is strictly concave, there is no loss of generality in restricting attention to quality levels that are only a function of suppliers' types. Let $q_{k}$ denote the quality level to be delivered by a type $k$ supplier. This, together with suppliers' risk neutrality, implies that suppliers' payoffs and thus behavior only depends on their expected probabilities of winning and their expected payment. Let $x_{k}$ be the probability of winning the contract conditional on being type $k$ and let $m_{k}$ the expected payment she receives. Finally, let $U_{k}$ denote type $k$ 's equilibrium expected utility. We have: $U_{k}=m_{k}-x_{k}\left(\theta_{1 k}+\theta_{2 k} q_{k}\right)$.

With these simplifications and notation, the buyer's expected utility from the mechanism is given by

$$
F\left(x_{k}, q_{k}, U_{k}\right)=N \sum_{k \in\{h H, l H, h L, l L\}} \alpha_{k}\left(x_{k} W_{k}\left(q_{k}\right)-U_{k}\right)
$$

The buyer seeks to maximize this expression over contracts $\left(x_{k}, q_{k}, U_{k}\right)$, subject to suppliers' incentive compatibility (IC) constraints:

$$
U_{k} \geq U_{j}+x_{j}\left(\theta_{1 j}-\theta_{1 k}\right)+x_{j} q_{j}\left(\theta_{2 j}-\theta_{2 k}\right) \quad \text { for all } k, j \in\{h H, l H, h L, l L\},
$$

individual rationality (IR) constraints:

$$
U_{k} \geq 0 \quad \text { for all } k \in\{h H, l H, h L, l L\},
$$

and subject to the feasibility constraint that the probability of awarding the contract to a subset of the types is always less than or equal to the probability of such types in the population:

$$
N \sum_{k \in K} \alpha_{k} x_{k} \leq 1-\left(1-\sum_{k \in K} \alpha_{k}\right)^{N} \text { for all subsets } K \text { of }\{h H, l H, h L, l L\}
$$

Finally, non exclusion imposes that

$$
\underset{k \in\{h H, l H, h L, l L\}}{N \sum_{k} x_{k}=1}
$$

Border (1991) guarantees that the feasibility constraint is both necessary and sufficient for the expected probabilities $x_{k}$ to be derived from a real allocation mechanism. This ensures that the solution to the maximization problem of (1) subject to (2), (3), (4) and (5) is implementable.

\footnotetext{
${ }^{3}$ Note that, unlike in environments with continuous multidimensional types (Armstrong, 1996), the assumption of non exclusion is not particularly restrictive in discrete type environments. It is easy to find parameter values such that all virtual welfares in the solution are positive making exclusion non-optimal (this can be seen for example in expression (6) below).
} 
The buyer's problem has four individual rationality constraints, 12 incentive compatibility constraints and 15 feasibility constraints. We can simplify them somewhat with the following results:

Lemma 1: Consider the feasibility constraints (4), and define an n-type constraint as a feasibility constraint with the relevant subset $K$ having $n$ elements. The following statements hold:

$i$. At most one one-type constraint binds, at most one two-type constraint binds and at most one three-type constraint binds.

ii. These binding constraints are nested, in the sense that the type in the binding one-type constraint must belong to the binding two-type constraint, and so on.

The proof of Lemma 1 is in the Appendix. The intuition is as follows. Suppose that, at the solution, the contract is allocated according to the following order of priority: $l L \succ l H \succ h L \succ h H$, i.e. give the contract to a type $l L$ if there is one, otherwise to a type $l H$ if there is one, and so on. This means that the ex-ante probability that a $l L$ type gets the contract is the probability that there is at least one type $l L$ suppliers among the $N$ suppliers, i.e. $N \alpha_{l L} x_{l L}=1-\left(1-\alpha_{l L}\right)^{N}$. Thus the one-type constraint binds for $l L$. It cannot bind for any other types because a binding constraint for another type would imply that that type has priority over all other types in the allocation, a contradiction. Next, $l L \succ l H \succ h L \succ h H$ also means that the contract is allocated to a type $l L$ or $l H$ whenever there is one among the $N$ suppliers. This means that the ex-ante probability of a type $l L$ or $l H$ winning, $N\left(\alpha_{l L} x_{l L}+\alpha_{l H} x_{l H}\right)$, is the probability that there is at least one of these types among the suppliers, $1-\left(1-\alpha_{l L}-\alpha_{l H}\right)^{N}$. Thus the two-type constraint binds for $\{l L, l H\}$, showing that the binding constraints are indeed nested. Statement (i) of Lemma 1 suggests that it could be the case that, say, no one-type constraint binds. This will be the case, for instance if the order of priority is $l L \sim l H \succ h L \succ h H$, that is, $l L$ and $l H$ have priority over all the other types, but if there are a $l L$ type and a $l H$ type, the buyer allocates the contract among them randomly. In this case, no one-type constraint binds. Finally, note that the suppliers' expected probabilities are weakly aligned with their order of priority in the sense that, if $k \succ j$, then $x_{k}>x_{j}$ but if $k \sim j$, then $x_{k} \gtreqless x_{j}$.

For future reference, denote the winning probabilities resulting from the efficient allocation $(l L \succ$ $h L \succ l H \succ h H)$ by $x_{k}^{F B}, k \in\{h H, l H, h L, l L\}$. Denote the winning probabilities for type $l H$ and $h L$ resulting from the allocation according to order of priority $l L \succ l H \succ h L \succ h H$ by $x_{l H}^{\max }$ and $x_{h L}^{\min }$.

Standard manipulation of the incentive compatibility constraints and the individual rationality constraints allows us to order the probabilities of winning in a limited way.

Lemma 2: At any solution, $x_{l H} \geq x_{h H}, x_{l L} \geq x_{h L}$ and $U_{h H}=0$

The key difficulty we face in characterizing the solution to the buyer's problem is in identifying the set of binding constraints at the optimum together with the associated partition of the parameter space. Our approach is to start with the buyer-optimal efficient mechanism. The buyer-optimal efficient mechanism is the mechanism that implements the efficient allocation in the way most 
favorable to the buyer. Efficiency requires that qualities are set such that $q_{l L}=q_{h L}=\underline{q}$ and $q_{h L}=q_{h H}=\bar{q}$, and that the probabilities are set equal to the first best probabilities, i.e. $x_{k}=x_{k}^{F B}$ for all $k$. Efficiency does not pin down payments to suppliers when private information is discrete. The buyer-optimal efficient mechanism (which we will simply refer to as "the efficient auction") sets payments to maximize the buyer's expected utility while satisfying all incentive compatibility constraints. In practice, only two sets of IC constraints bind at the efficient auction, as the next lemma establishes:

Lemma 3: When $\Delta \theta_{1}>\Delta \theta_{2} \bar{q}, I C_{l H, h H}, I C_{h L, h H}$ and $I C_{l L, h L}$ bind in the efficient auction. When $\Delta \theta_{1}<\Delta \theta_{2} \bar{q} I C_{l H, h H}, I C_{h L, h H}$ and $I C_{l L, h L}$ bind (see Figure 1).

Insert Figure 1 Here

The proof of Lemma 3 can be found in the Appendix. From this starting point, we progressively increase the buyer's expected utility by adjusting the conditional probabilities of winning (the $x$ 's) and the qualities (the $q$ 's) until there is no further scope for improvement. At this point, we will have reached the global maximum as guaranteed by the next lemma. Moreover, this approach ensures that we cover the entire parameter space.

Lemma 4: The first order conditions of the maximization problem (1) subject to (2), (3), (4) and (5) are necessary and sufficient for a global maximum.

The proof of Lemma 4 is in the Appendix. It allows us to prove the main result of this section:

Theorem 1: Characterization of the optimal buying mechanism

Define $q_{h H}^{2}=\arg \max _{q}\left\{W_{h H}(q)-\frac{\alpha_{h L}+\alpha_{l L}}{\alpha_{h H}} q \Delta \theta_{2}\right\}$ and $q_{l H}^{2}=\arg \max _{q}\left\{W_{l H}(q)-\frac{\alpha_{h L}+\alpha_{l L}}{\alpha_{l H}} q \Delta \theta_{2}\right\}$. Part I: When $\Delta \theta_{1}-\Delta \theta_{2} \bar{q} \geq 0$, the probabilities of winning and quality levels in the optimal buying mechanism are as given in Table 1.

Part II: When $\Delta \theta_{1}-\Delta \theta_{2} \bar{q}<0$, the probabilities of winning and quality levels in the optimal buying mechanism are as given in Table 2.

Insert Tables $1 \& 2$ Here

Sketch of Proof: The full proof of Theorem 1 is very long (18 pages). Here we only provide a proof for solutions 1.1.a and 1.1.b to illustrate our approach to deriving the full characterization. The reader is referred to Asker and Cantillon (2005) for the full proof.

Consider the efficient auction. Let $U_{k, j}$ be the expected utility of a type $k$ pretending she is of type $j$. To ensure incentive compatibility, while minimizing suppliers' rents, suppliers' expected utilities in the efficient auction must be set such that $U_{k}=\max _{j \neq k} U_{k, j}$. $U_{h H}=0$ by Lemma 2 . 
From Lemma 4, we need to consider only two cases. If $\Delta \theta_{1}-\Delta \theta_{2} \bar{q} \geq 0$, the per-supplier buyer's expected utility in the efficient auction, $\sum_{k} \alpha_{k}\left[x_{k}^{F B} W_{k}\left(q_{k}\right)-U_{k}\right]$, is given by:

$$
\begin{aligned}
& \alpha_{l H} x_{l H}^{F B} W_{l H}\left(q_{l H}\right)-\alpha_{l H} x_{h H}^{F B} \Delta \theta_{1}+\alpha_{h H} x_{h H}^{F B} W_{h H}\left(q_{h H}\right)+\alpha_{h L} x_{h L}^{F B} W_{h L}\left(q_{h L}\right)-\alpha_{h L} x_{h H}^{F B} q_{h H} \Delta \theta_{2} \\
& +\alpha_{l L} x_{l L}^{F B} W_{l L}\left(q_{l L}\right)-\alpha_{l L} x_{h L}^{F B} \Delta \theta_{1}-\alpha_{l L} x_{h H}^{F B} q_{h H} \Delta \theta_{2}
\end{aligned}
$$

(where all qualities are initially equal to the first best qualities) or, to highlight the virtual welfare generated by each supplier:

$$
\begin{aligned}
& \alpha_{l H} x_{l H}^{F B} W_{l H}\left(q_{l H}\right)+\alpha_{h H} x_{h H}^{F B}\left[W_{h H}\left(q_{h H}\right)-\frac{\alpha_{l H}}{\alpha_{h H}} \Delta \theta_{1}-\frac{\alpha_{h L}+\alpha_{l L}}{\alpha_{h H}} q_{h H} \Delta \theta_{2}\right] \\
& +\alpha_{h L} x_{h L}^{F B}\left[W_{h L}\left(q_{h L}\right)-\frac{\alpha_{l L}}{\alpha_{h L}} \Delta \theta_{1}\right]+\alpha_{l L} x_{l L}^{F B} W_{l L}\left(q_{l L}\right)
\end{aligned}
$$

The rents of suppliers $l L$ and $h L$ depend positively on $q_{h H}$ and the buyer can increase his expected utility by decreasing $q_{h H}$, ideally until

$$
q_{h H}^{2}=\arg \max _{q_{h H}}\left\{W_{h H}\left(q_{h H}\right)-\frac{\alpha_{l H}}{\alpha_{h H}} \Delta \theta_{1}-\frac{\alpha_{h L}+\alpha_{l L}}{\alpha_{h H}} q_{h H} \Delta \theta_{2}\right\}
$$

Suppose no new IC constraint binds in the process. (This will be the case if $x_{l H}^{F B}\left[\Delta \theta_{1}-\Delta \theta_{2} \bar{q}\right]>$ $\left.x_{h H}^{F B}\left[\Delta \theta_{1}-\Delta \theta_{2} q_{h H}^{2}\right]\right)$. Now consider again (6). There is no further scope for improvement by distorting qualities. Furthermore, the virtual welfare of $l L$ is clearly the largest of all so that it is optimal to set $x_{l L}=x_{l L}^{F B}$. However, the relative ranking of the virtual welfare of $l H$ and $h L$ is unclear. If $W_{h L}(\underline{q})-\frac{\alpha_{l L}}{\alpha_{h L}} \Delta \theta_{1}>W_{l H}(\bar{q})$, the virtual welfare generated by supplier $h L$ remains larger than that of $l H$ so the optimal allocation is the first best allocation. This is solution 1.1.a.

Suppose instead that the virtual welfare associated with $l H$ is larger than that associated with $h L$, itself larger than the virtual welfare associated with $h H$ (formally, and referring to $(6), W_{l H}(\bar{q})>$ $\left.W_{h L}(\underline{q})-\frac{\alpha_{l L}}{\alpha_{h L}} \Delta \theta_{1} \geq W_{h H}\left(q_{h H}^{2}\right)-\frac{\alpha_{l H}}{\alpha_{h H}} \Delta \theta_{1}-\frac{\alpha_{h L}+\alpha_{l L}}{\alpha_{h H}} q_{h H}^{2} \Delta \theta_{2}\right)$. In this case, the buyer would rather give the contract to supplier $l H$ than to supplier $h L$, i.e. he would like to change the order of priority in the allocation. Increasing $x_{l H}$ while decreasing $x_{h L}$ concurrently (keeping $\alpha_{l H} x_{l H}+\alpha_{h L} x_{h L}+\alpha_{l L} x_{l L}^{F B}$ constant) does not initially affect any of the virtual welfare and it increases the buyer's expected utility. This process continues until either a new IC constraint binds or we have reached the feasibility constraint for $x_{l H}: N\left(\alpha_{l H} x_{l H}^{\max }+\alpha_{l L} x_{l L}^{F B}\right)=1-\left(\alpha_{h L}+\alpha_{h H}\right)^{N}$. Suppose we reach $x_{l H}=x_{l H}^{\max }$ before any new IC constraint binds. The qualities and probabilities are then all optimized given the binding constraints. This corresponds to solution 1.1.b. Solution 1.1.c. arises if a new IC constraint binds in the process. Solutions 1.1.d. and 1.1.e. arise when the ordering of virtual social welfares is such that type $l H$ is preferred to type $h H$ which, in turn, is preferred to type $h L$. End of the sketch of the proof.

Tables 1 and 2 present the main features of the solution. The second column describes the probabilities of winning and the last four columns describe the qualities at the solution (an interval means that the optimal level of quality lies in this interval). For instance, Solution 1.2.b has $x_{l L}=x_{l L}^{F B}$ 
which is greater than $x_{h L}\left(<x_{h L}^{F B}\right)$. This is in turn greater than $x_{l H}\left(>x_{l H}^{F B}\right)$ and $x_{h H}=x_{h H}^{F B}$. Both $q_{l L}$ and $q_{h L}$ are at the first best levels and $q_{h H} \in\left(q_{h H}^{2}, \bar{q}\right)$ and $q_{l H} \in\left(q_{l H}^{2}, \bar{q}\right)$. Both are distorted below the first best level. The conditions that define each solution depend on the resulting binding constraints and virtual welfares as summarized in Figures 2 and 3 . The value of the objective function and the value of the control variables at the solution are continuous in the parameters of the model.

\section{Insert Figure 2 Here}

The following patterns emerge from the tables. First, the solution describing the optimal scheme depends on the number of suppliers as well as the usual parameters of the environment (distributions of types and cost structure). The reason is that the number of bidders affects the probabilities of winning and thus which incentive compatibility constraints bind. The dependence of the optimal scheme on the number of bidders is typical of multidimensional environments where the binding IC constraints are endogenous. No such effect is present in one-dimensional environments (Laffont and Tirole, 1987).

Second, there is some downward distortion in the quality provided by the high cost marginal suppliers. The quality provided by the low marginal cost suppliers is never distorted.

Third, probabilities of winning are also often distorted. Specifically, the probabilities of winning of the high marginal cost suppliers are sometimes distorted upwards, whereas the probability of winning of low marginal cost supplier $h L$ is sometimes distorted downwards. The allocation of supplier $l L$ is never distorted.

\section{Insert Figure 3 Here}

Putting these two last aspects together - productive and allocative distortions - we find no systematic "bias against quality" in the two-dimensional model, unlike in the one-dimensional model (Laffont and Tirole, 1987 and Che, 1993). While the economic conclusions differ, the underlying economic motivation is the same: reducing suppliers' rents. The qualities of the high marginal cost types are distorted downwards to reduce the low marginal cost supplier's benefit from imitating them. As illustrated in Figures 3 and 4, all binding constraints between suppliers with different marginal costs are from the low marginal cost supplier to the high marginal cost supplier so this "trick" is effective. This is also the case in the one-dimensional model where the distortion of high-cost types' quality lowers the informational rents of the low cost types. Similarly, the reason why supplier $h L$ 's probability of winning is sometimes below her first best level is to reduce supplier $l L$ 's rent. In each case, the optimal level of distortion balances a trade-off between the costs in terms of lost social welfare and the benefits in terms of reduced rents. 
The optimal scheme has two disadvantages. First, it depends finely on the parameters of the environment. Second, it is complex and does not seem to be implementable using a transparent procedure, a point on which we elaborate below. Yet, transparency is desirable and even often a requirement, as in public procurement. This suggests that, for practical purposes, second best solutions that are simple and robust performers in a variety of settings are likely to be more useful. Commonly used procedures are obvious candidates. They include scoring auctions, priceonly auctions with minimum quality standards, beauty contests, menu auctions where suppliers can submit several price-quality offers, and negotiation. Asker and Cantillon (2006) have shown that scoring auctions yield a higher expected utility to the buyer than a price-only auction with minimum standards and beauty contests, and that they dominate menu auctions when a second price or an ascending format is used. Hence, our contenders for second best procedures are scoring auctions and negotiation. We analyze these procedures in the next sections.

\section{Scoring Auctions}

In a scoring auction, the buyer announces a scoring rule that is linear in price, $S(p, q)=\widetilde{v}(q)-p$ (with $\widetilde{v}_{q} \geq 0, \widetilde{v}_{q q} \leq 0$ and $\max \widetilde{v}(q)-\theta_{2 i} q$ admitting a single interior solution), suppliers submit pricequality bids $(p, q)$, and the winner is the supplier whose bid generates the highest score according to the scoring rule. ${ }^{4}$ The winner's resulting obligation depends on the auction format. For example, in a first score scoring auction, the winner must deliver a quality level at a price that matches the score of his bid. In a second score scoring auction, the winner must deliver a quality level at a price that matches the second highest score submitted. Scoring auctions are increasingly used in public and private procurement and are supported by several procurement software packages (see, Asker and Cantillon, 2006 for examples and references).

\subsection{Theoretical properties}

Scoring auctions put some structure on suppliers' bidding behavior. First, given a scoring rule $\widetilde{v}(q)-p$, suppliers choose their bids to maximize the score they generate given their profit target $\pi$, i.e. they solve $\max _{(p, q)}\{\widetilde{v}(q)-p\}$ subject to $p-\theta_{1 i}-\theta_{2 i} q=\pi$. Substituting for $p$ inside the maximizer yields

$$
\max _{q}\left\{\widetilde{v}(q)-\theta_{1 i}-\theta_{2 i} q-\pi\right\}
$$

A property of the solution is that it is independent of $\pi$, the profit target, and of $\theta_{1 i}$, the fixed cost. Second, a standard incentive compatibility argument establishes that the ordering of suppliers' winning probabilities must correspond to their ability to generate a higher score (intuitively, define $\max _{q}\left\{\widetilde{v}(q)-\theta_{1 i}-\theta_{2 i} q\right\}$ as the supplier's type). Thus, a scoring auction will implement a particular allocation if two conditions hold:

\footnotetext{
${ }^{4}$ Asker and Cantillon (2006) refer to this auction format as a quasilinear scoring auction to emphasize the linearity of the scoring rule in $p$.
} 
1. [production constraint] Given the scoring rule, suppliers maximize (7) by choosing the level of quality assigned by the allocation.

2. [ranking constraint] The ranking of $\max _{q}\left\{\widetilde{v}(q)-\theta_{1 i}-\theta_{2 i} q\right\}$ and, thus, the ranking of the scores is consistent with the assigned probabilities of winning.

The next Theorem characterizes the set of allocations that can be implemented by a scoring auction.

Theorem 2: An allocation can be implemented with a scoring auction if and only if (1) $q_{l H}=q_{h H}$, $q_{h L}=q_{l L}$ with $q_{l H}, q_{h H}<q_{h L}, q_{l L}$, (2) $\alpha_{l H} x_{l H}+\alpha_{h L} x_{h L}=\alpha_{l H} x_{l H}^{F B}+\alpha_{h L} x_{h L}^{F B}, x_{h H}=x_{h H}^{F B}$ and $x_{l L}=x_{l L}^{F B}$, (3) $\Delta \theta_{1}-\Delta \theta_{2} q_{h L} \leq 0$ when $x_{h L}>x_{h L}^{\min }$ and (4) $\Delta \theta_{1}-\Delta \theta_{2} q_{l H} \geq 0$ whenever the allocation is such that $x_{l H}>x_{l H}^{F B}$.

Theorem 2 clarifies the constraints that a scoring auction places on the possible allocations. Its proof can be found in the Appendix. The first condition says that two suppliers with the same marginal cost of quality must be providing the same level of quality. Moreover, suppliers with a lower marginal cost of quality must deliver higher levels of quality at equilibrium. These two properties follow from the structure of (7). The second condition says that, at equilibrium, type $l L$ must win over any other type, and that type $h H$ must lose against any other type. The reason is that type $l L$ generates the highest value for $\max _{q}\left\{\widetilde{v}(q)-\theta_{1 i}-\theta_{2 i} q\right\}$ for any scoring rule and that type $h H$ generates the lowest such value. The third and fourth conditions follow from the combination of the production constraint and the ranking constraint. Finally, to prove the sufficiency part of the claim, we construct a scoring rule that implements the allocation under conditions (1) through (4).

An immediate consequence of Theorem 2 is that the efficient auction can be implemented by a scoring auction. Such a scoring auction has a scoring rule that corresponds to the buyer's preferences and uses a second score format. ${ }^{5}$ It is both robust, in the sense that it does not depend on the parameters of the environment, and transparent.

Theorem 2 also clarifies why scoring auctions cannot in general implement the optimal solution. First, $q_{h H}$ and $q_{l H}$ differ generically in the optimal mechanism. Moreover, the solution requires $x_{h H}>x_{h H}^{F B}$ in several cases. This said, scoring auctions have two potential advantages over the efficient auction. First, they allow for distortion in production. Second, they allow some distortion in allocation probabilities in the same direction as the optimal procedure. The next section investigates these properties numerically.

\footnotetext{
${ }^{5}$ To ensure that the scoring auction generates as much utility to the buyer as possible, type-specific down-payments must be included. These down-payments are an artifact of the discrete type space. They maintain incentive compatibility and increase the buyer's utility.
} 


\subsection{Computational Results}

Having identified the constraints that scoring auctions place on allocations, we next turn to the question of their relative performance. We interpret the difference between the expected utility generated from the optimal mechanism and the expected utility from the efficient auction as the surplus available to a strategic buyer, and ask to what extent scoring auctions capture this surplus. We answer this question in two steps. First, we compute a tight upper bound to the performance of scoring auctions by computing the expected utility generated by the optimal scoring auction. Second, we analyze the performance of naïve scoring auctions, that is, scoring auctions that use scoring rules that differ from the optimal scoring rule in an arbitrary way.

\subsubsection{Bounds}

To evaluate the performance of scoring auctions, we compute an upper bound to the expected utility that scoring auctions generate by adding the constraints of Theorem 2 onto the initial problem and solving the resulting program numerically. The resulting expected utility is then compared with the expected utility from the efficient auction and the expected utility from the optimal mechanism.

\section{Insert Figure 4 Here}

Figure 4 shows the results for an environment where $v(q)=3 \sqrt{q}, l=L=1, h=2, N=2$ and $\alpha_{k}=0.25$. The value of $\Delta \theta_{1}$ varies along the $x$-axis. The expected utlity from the optimal mechanism lies above that from the optimal scoring auction which, in turn, dominates that from the efficient auction. As the value of $\Delta \theta_{1}$ increases, the expected utility decreases in all three mechanisms. This is to be expected. When $\Delta \theta_{1}$ increases, the maximum level of welfare decreases because suppliers' costs increase. Moreover, fixed costs become relatively more important as a source of adverse selection. The kink at the point $\Delta \theta_{1}=0.5625$ corresponds to the point when the binding incentive compatibility constraint for type $h L$ in the efficient mechanism switches from $\mathrm{IC}_{h L, l H}$ to $\mathrm{IC}_{h L, h H}$ (thus $\Delta \theta_{1}=\Delta \theta_{2} \bar{q}$ ). The resulting increases in the weight of $\Delta \theta_{1}$ in the buyer's expected utility explains the kink. ${ }^{6}$

We replicated this exercise for a range of environments by varying the values for the $\alpha_{k}$ 's and some of the other parameters of the model. Table 3 reports the results. The third column reports the

\footnotetext{
${ }^{6}$ As $\Delta \theta_{1}$ tends to 0 , the source of adverse selection reduces to one dimension, the marginal cost. In this case, Che (1993) has shown that a scoring auction implements the optimal mechanism. The reason why the expected utility from the optimal scoring auction does not converge to the expected utility of the optimal mechanism in our graph is that there is some discontinuity in the optimal scoring auction at $\Delta \theta_{1}>0$. As long as $\Delta \theta_{1}>0$, scoring auctions impose that $l H$ generates a strictly higher score than $h H$. Thus $x_{l H} \geq x_{l H}^{F B}>x_{h H}=x_{h H}^{F B}$ (Theorem 2). This leaves some informational rent to $l H$ and increases the rents of $h L$ and $l L$ relative to the case where $x_{l H}=x_{h H}$. When $\Delta \theta_{1}=0$, suppliers $l H$ and $h H$ are essentially the same. The optimal scoring auction will thus set $x_{l H}=x_{h H}$ and leave no rent to supplier $l H$.
} 
average captured surplus over the full range of values that $\Delta \theta_{1}$ can take. The fourth column reports the maximum percentage of the surplus that the optimal scoring auction captures together with the corresponding value of $\Delta \theta_{1}$. The fifth column does the same for the worst relative performance of the optimal scoring auction. Finally, columns 6 and 7 report the percentage of $\Delta \theta_{1}$ values for which the performance of the optimal scoring auction is greater than $80 \%$ (column 6 ) or within 10 percentage points of its worst performance (column 7). For the core set of experiments (experiments 1 through 20), $v(q)=a q^{b}$ with $a=3$ and $b=0.5, l=L=1, h=2, N=2$. The bottom part of the table considers other values for $a, b, L$ and $H$. (We keep $N=2$ in all our experiments because this is where the actual choice of mechanisms is likely to matter most). Figure 5 shows the relative performance of the optimal scoring auction as $\Delta \theta_{1}$ changes for selected probability configurations.

\section{Insert Table 3 Here, Insert Figure 5 Here}

The results are the following. First, in every experiment, there exists a value of $\Delta \theta_{1}$ for which the optimal scoring auction does as well as the optimal mechanism. Second, the point at which this occurs seems somewhat persistent across environments. Third, the optimal scoring auction captures on average more than two thirds of the surplus from being strategic, even though this proportion can dip down to $20 \%$ for some values of $\Delta \theta_{1}$ and some environments. Fourth, the optimal scoring auction does poorly when the fixed cost and the marginal cost are negatively correlated. We now investigate each of these points in more detail.

In every experiment, there exists a value of $\Delta \theta_{1}$ for which the optimal scoring auction does as well as the optimal mechanism. Given Theorem 2, this must happen at parameter values such that there are binding incentive compatibility constraints directed to both $l H$ and $h H$ from low marginal cost suppliers in the optimal scheme (otherwise there is no chance that the qualities provided by suppliers $l H$ and $h H$ are the same in the optimal scheme). Inspection of Table 1 and 2 suggests that the only candidates consistent with implementation with a scoring auction are solutions 1.1.c., 1.2.a, 1.2.b or 1.2.c. (recall that scoring auctions require $x_{h H}=x_{h H}^{F B}$ ). Closer inspection of the numerical solution suggests that the maximum performance of the optimal scoring auction happens when the optimal mechanism corresponds to solutions either 1.2.a, or 1.2.c.

An inspection of Figure 5 and the results in Table 3 suggest the optimum is reached at a similar region in each set of simulations (in particular this point is always less than 0.5625 , the point when the binding incentive compatibility constraint for type $h L$ in the efficient mechanism switches from $\mathrm{IC}_{h L, l H}$ to $\left.\mathrm{IC}_{h L, h H}\right)$. This begs question of why there and not elsewhere? The parameter setting in figure 5 where the probabilities are $(35,35,15,15)$ suggests that it is possible for the optimum revenue to be generated to the right of the dip in revenue. To investigate this we ran a set of experiments for the probabilities $(40,40,10,10)$ and $(45,45,5,5)$ - experiments 17 and 18 . In this setting the solution corresponds to solution 1.1.d in table 1. In experiment 17, at $\Delta \theta_{1}=0.93375$, the optimal scoring auction captures $99.03 \%$ of the available strategic surplus, whereas in experiment 
17 , at $\Delta \theta_{1}=1.02375$, this is raised to $99.89 \%$. While it appears that the scoring auction does well in these regions it falls short of the optimu mechanism because of the restriction that $x_{h H}=x_{h H}^{F B}$ in the scoring auction.

It has been noted that the point at which the optimal scoring auction does as well as the optimal scheme is constant across cases 1-5 and 10-15. After further inspection, this is an artifact of the common symmetric structure of these parameter settings. These settings are such that $\alpha_{l L}=\alpha_{h H}$ and $\alpha_{h L}=\alpha_{l H}$. A comparison with experiments 6 and 19 illustrates this point: In experiment 6 the probability are such that $\alpha_{l L}=\alpha_{h L}>\alpha_{l H}=\alpha_{h H}$ resulting in a move in the location of the optimum; experiment 19 makes a similar perturbation, with the additional shock that $\alpha_{l H}<\alpha_{h H}$. The optimal scoring auction does very well overall. It captures, on average, more than two thirds of the surplus, and in ten of the 13 core experiments reported in Table 3, it captures more than $80 \%$ of the surplus for the majority of the values $\Delta \theta_{1}$ can take. This excellent performance seems attributable to the relative flexibility scoring auctions leave in terms of allocation.

Table 3 and Figure 5 also indicate that scoring auctions perform less well in some environments. This poor performance tends to happen around the point where there is a kink in the expected utility of the efficient auction. This coincides with the point at which both incentive compatibility constraints out of type $h L$ are close to being binding in the efficient mechanism (one must bind, and the other is 'close' to binding). As a result, those IC constraints leave little scope for rent extraction before they bind. Given that the scoring auction is less flexible in the face of these constraints than the optimal mechamism it is not surprising that its relative performance suffers.

Similarly, negative correlation between the marginal cost and the fixed costs decreases the performance of the optimal scoring auction (see experiments 4, 5 and 15). Intuitively, negative correlation moves the environment further from the one-dimensional environment for which scoring auctions are known to do well (Che, 1993). The weight of types $l H$ and $h L$ is large in the total expected utility of the buyer, and so the gains from distorting quality tends to make $q_{h H}$ far from being firstbest. In light of this, it is noteworthy that positive correlation does not unambiguously generate strong performances from the scoring auction. In experiments 2 and 3 , where the extent of positive correlation is increasing, the scoring auction appears to be doing increasingly well. However, in experiment 14 , this trend does not continue. What is happening here is that the extra flexibility in the optimal mechanism is able to exploit the environment as it moves toward the one-dimensional case far sooner than the scoring auction. The relative performance of the scoring auction in experiment 14 reflects a reconfiguration of the optimal mechanism in the face of the changing environment, rather than any significant change in the scoring auction itself.

\subsubsection{Simple manipulations of the scoring rule}

While consideration of the optimal scoring auction is fruitful, it remains an upper bound. Moreover, the optimal scoring auction also depends finely on the parameters of the environment. For this 
reason, we also investigate the performance of "naïve scoring auctions." Naïve scoring auctions are scoring auctions that use simple scoring rules that differ from the true preferences of the buyer in an arbitrary way (but in the same direction as recommended by the optimal scheme.

Suppose the buyer's utility is given by $v(q)=a q^{b}$. We consider the expected utility generated by a scoring auction with scoring rule $a^{*} q^{b}-p$ and $a^{*}=(0.9) a$ or $a=(0.95) a$. Thus the winner is the supplier who generates the highest value for $\max _{q}\left\{a^{*} q^{b}-\theta_{1 i}-\theta_{2 i} q\right\}$ and she receives the value of the second highest score. ${ }^{7}$

\section{Insert Figure 6 Here, Insert Table 4 Here}

Figure 6 shows the proportion of the strategic surplus captured by the naïve scoring auction when $a^{*}=(0.95) a$ and $\alpha_{k}=0.25$. At first, the proportion of surplus is at $14 \%$ and then increases up to $38 \%$. At this point the binding IC constraints change in the naïve scoring auction, $I C_{h L, h H}$ ceases to bind and $I C_{h L, l h}$ starts binding. This causes a sharp drop in the proportion of surplus extracted by the naïve scoring auction (a phenomenon similar to the drop in the performance of the optimal scoring auction at that point). The next major shift is the dramatic increase in the proportion of surplus captured when $\Delta \theta_{1} \simeq 1$. The reason this happens is because the naïve scoring auction distorts the probabilities of winning at this point (by giving priority to type $l H$ over type $h L$ ). Because the virtual welfare (as measured using to the buyer's true utility function) generated by type $l H$ is also higher than the virtual welfare generated by type $h L$ at this point, this increases expected utility sharply. Note that this reversal in winning probabilities (or more precisely the flexibility of the optimal scoring auction to pick a range of $x_{l H}, x_{h L}$ consistent with requirement 2 of Theorem 2) explains why the optimal scoring auction's performance improves from $\Delta \theta_{1} \simeq 0.5625$ onwards.

Table 4 summarizes the results of this exercise repeated for the parameters of the core experiments. The qualitative results in Figure 6 are replicated in all experiments. Table 4 reports the range of performance for the naïve scoring auction, when it allocates the contract in the same way as the efficient auction does (column 4), and when it allocates the contract differently by giving priority to type $l H$ over type $h L$. The results show that the naïve scoring auction does particularly well when it distorts the allocation probabilities. This suggests that distortions in the allocation probabilities are more important than distortions in qualities for rent extraction purposes.

\section{Sequential procedures}

We now turn to sequential procedures. Sequential procedures are mechanisms that approach suppliers at most once. Several practical procedures belong to this category including many models of negotiation. These include a procedure in which the buyer negotiates with a single supplier and

\footnotetext{
${ }^{7}$ As before some adjustments need to be made to account for the discreteness of the types space.
} 
walks out if the supplier does not offer him a certain level of utility, a procedure whereby the buyer makes sequential take-it-or-leave-it price offers (as in Manelli and Vincent, 1995) or a procedure where the buyer pre-selects a single supplier and offers him a menu of optimal screening contracts (as in Wang, 1998).

\subsection{Theoretical properties}

Our purpose here is to get a sense of the costs and benefits of sequential procedures relative to the efficient auction and the optimal procedure. To do this, we characterize the optimal sequential procedure, i.e. the mechanism that maximizes the buyer's expected utility conditional on approaching suppliers at most once. This optimal sequential procedure provides an upper bound to what sequential procedures can achieve because it gives all bargaining power to the buyer.

The optimal sequential procedure solves a simple dynamic programming problem. The buyer approaches suppliers one at a time and offers them a menu of optimal screening contracts that takes into account the number of remaining suppliers. ${ }^{8}$ Because suppliers are approached only once, their dominant strategy is to accept the best offer that meets their individual rationality constraint. If a supplier refuses, the buyer turns to the next supplier. To ensure that we can compare our results with the results of the previous sections, we require that the contract is allocated with probability one which implies that the offers made in the last round must be acceptable by all types of suppliers. ${ }^{9}$ The next Lemma shows that, in any round, we can without loss of generality consider a menu of two contracts, one targeted at the low marginal cost suppliers, and the other targeted at the high marginal cost suppliers. The intuition is that contracts of the form $(p, q)$ are unable to screen over suppliers' fixed cost, $\theta_{1}$.

Lemma 5: In any round, the buyer offers at most two contracts.

Proof: Towards a contradiction, suppose the buyer offers three contracts and, without loss of generality, suppose that one, $\left(p_{l L}, q_{l L}\right)$, is targeted at $l L$ and another, $\left(p_{h L}, q_{h L}\right)$ is targeted at $h L$. Incentive compatibility requires:

$$
\begin{aligned}
p_{l L}-\underline{\theta}_{1}-\underline{\theta}_{2} q_{l L} & \geq p_{h L}-\underline{\theta}_{1}-\underline{\theta}_{2} q_{h L} \\
p_{h L}-\bar{\theta}_{1}-\underline{\theta}_{2} q_{h L} & \geq p_{l L}-\bar{\theta}_{1}-\underline{\theta}_{2} q_{l L}
\end{aligned}
$$

Thus $p_{l L}-\underline{\theta}_{2} q_{l L}=p_{h L}-\underline{\theta}_{2} q_{h L}$ and the contracts lie on an isoprofit locus for suppliers $h L$ and $l L,\left\{(p, q): p_{l L}-\underline{\theta}_{2} q_{l L}=p_{h L}-\underline{\theta}_{2} q_{h L}\right\}$. Since the buyer has strictly convex preferences, there is a unique contract on this locus that maximizes his utility. Q.E.D.

Let $\left(p_{1}^{n}, q_{1}^{n}\right)$ and $\left(p_{2}^{n}, q_{2}^{n}\right)$ denote the menu of contracts offered when $n$ suppliers remain, with the convention that $q_{1}^{n} \leq q_{2}^{n}$. In the last round, the buyer's optimization problem is given by

\footnotetext{
${ }^{8}$ In that sense, the optimal sequential procedure combines the features of Wang (1998)'s single-buyer single-supplier optimal negotiation and those of Manelli and Vincent (1995)'s sequential model of negotiation.

${ }^{9}$ As for the optimal procedure, such requirement can be perfectly consistent with optimality if the buyer values the good sufficiently highly. In that case, exclusion in the last period is suboptimal.
} 


$$
\max _{\left(p_{1}, q_{1}\right),\left(p_{2}, q_{2}\right)}\left(\alpha_{l H}+\alpha_{h H}\right)\left(v\left(q_{1}\right)-p_{1}\right)+\left(\alpha_{h L}+\alpha_{l L}\right)\left(v\left(q_{2}\right)-p_{2}\right)
$$

subject to suppliers' IR and IC constraints. Following standard arguments, supplier $h H$ 's IR constraint is binding, i.e. $p_{1}=\bar{\theta}_{1}+\bar{\theta}_{2} q_{1}$, and only the downward IC constraint is binding, i.e. $p_{2}-\underline{\theta}_{2} q_{2}=p_{1}-\underline{\theta}_{2} q_{1}$. Substituting for $p_{1}$ and $p_{2}$ in the objective function yields:

$$
\max _{\left(p_{1}, q_{1}\right),\left(p_{2}, q_{2}\right)}\left(\alpha_{l H}+\alpha_{h H}\right)\left(v\left(q_{1}\right)-\bar{\theta}_{1}-\bar{\theta}_{2} q_{1}\right)+\left(\alpha_{h L}+\alpha_{l L}\right)\left(v\left(q_{2}\right)-\bar{\theta}_{1}-\underline{\theta}_{2} q_{2}-\Delta \theta_{2} q_{1}\right)
$$

This establishes Lemma 6:

Lemma 6: The optimal take-it-or-leave-it offer in the last round is given by $\left(p_{1}^{1}, q_{1}^{1}\right)$, where $p_{1}^{1}=\bar{\theta}_{1}-\bar{\theta}_{2} q_{1}^{1}$ and $q_{1}^{1}=\arg \max \left\{v(q)-\bar{\theta}_{2} q-\frac{\left(\alpha_{h L}+\alpha_{l L}\right)}{\left(\alpha_{l H}+\alpha_{h H}\right)} q \Delta \theta_{2}\right\}$, and $\left(p_{2}^{1}, q_{2}^{1}\right)=\left(p_{1}^{1}+\underline{\theta}_{2}\left(\underline{q}-q_{1}^{1}\right), \underline{q}\right)$. The resulting continuation value is given by $\left(\alpha_{l H}+\alpha_{h H}\right) W_{h H}\left(q_{1}^{1}\right)+\left(\alpha_{h L}+\alpha_{l L}\right)\left(W_{h L}^{F h H}(\underline{q})-\Delta \theta_{2} q_{1}^{1}\right)$.

Let $V_{n}$ denote the continuation value of a buyer facing $n$ remaining bidders. We are now ready to characterize the optimal sequential procedure:

Theorem 3 (characterization of the optimal sequential procedure): The optimal sequential procedure is such that the buyer offers the menu of contracts defined in Lemma 6 in the last round and, when $n>1$, one of the four following pairs of contracts, depending on which one yields the largest continuation value (conditional on the condition in the third column being satisfied):

\begin{tabular}{lll}
\hline$K_{n}$ & Offers $\left(p_{1}^{n}, q_{1}^{n}\right)$ and $\left(p_{2}^{n}, q_{2}^{n}\right)$ & $V_{n}$ \\
\hline \hline$l L$ & $\left(\underline{\theta}_{1}+\underline{\theta}_{2} \underline{q}, \underline{q}\right)$ & $\alpha_{l L} W_{l L}^{F B}+\left(1-\alpha_{l L}\right) V_{n-1}$ \\
\hline$l L, l H$ & $\left(\underline{\theta}_{1}+\bar{\theta}_{2} q_{1}^{*}, q_{1}^{*}\right)$ & $\alpha_{l L}\left(W_{l L}^{F B}-\Delta \theta_{2} q_{1}^{*}\right)+$ \\
& $\left(\underline{\theta}_{1}+\underline{\theta}_{2} \underline{q}+\Delta \theta_{2} q_{1}^{*}, \underline{q}\right)$ & $\alpha_{l H} W_{l H}\left(q_{1}^{*}\right)+\left(\alpha_{h L}+\alpha_{h H}\right) V_{n-1}$ \\
& $q_{1}^{*}=\arg \max \left\{v(q)-\bar{\theta}_{2} q-\frac{\alpha_{l L}}{\alpha_{l H}} \Delta \theta_{2} q\right\}$ & Condition: $\Delta \theta_{1}-\Delta \theta_{2} q_{1}^{*} \geq 0$ \\
\hline$l L, h L$ & $\left(\bar{\theta}_{1}+\underline{\theta}_{2} \underline{q}, \underline{q}\right)$ & $\left(\alpha_{l L}+\alpha_{h L}\right) W_{h L}^{F B}+\left(\alpha_{l H}+\alpha_{h H}\right) V_{n-1}$ \\
\hline$l L, l H, h L$ & $\left(\underline{\theta}_{1}+\bar{\theta}_{2} q_{1}^{* *}, q_{1}^{* *}\right)$ & $\left(\alpha_{l L}+\alpha_{h L}\right)\left(W_{l L}^{F B}-\Delta \theta_{2} q_{1}\right)$ \\
& $\left(\underline{\theta}_{1}+\underline{\theta}_{2} \underline{q}+\Delta \theta_{2} q_{1}^{* *}, \underline{q}\right)$ & $\alpha_{l H} W_{l H}\left(q_{1}^{* *}\right)+\alpha_{h H} V_{n-1}$ \\
& $q_{1}^{* *}=\arg \max \left\{v(q)-\bar{\theta}_{2} q-\frac{\left(\alpha_{l L}+\alpha_{h L}\right)}{\alpha_{l H}} \Delta \theta_{2} q\right\}$ & Condition: $\Delta \theta_{1}-\Delta \theta_{2} q_{1}^{* *} \leq 0$ \\
\hline
\end{tabular}

(The first column in the table indicates the set of supplier types who will accept the buyer's offer when $n$ suppliers remain, and the third column indicates the buyer's continuation value, $\left.V_{n}\right)$.

(Suppliers of types $l H$ and $h H$ accept the contract $\left(p_{1}^{n}, q_{1}^{n}\right)$ if it satisfies their IR constraint, and likewise, suppliers of types $l L$ and $h L$ accept contract $\left(p_{2}^{n}, q_{2}^{n}\right)$ if it satisfies their IR constraint).

Proof: See Appendix

Note that $q_{1}^{* *}<q_{1}^{*}$, so that $K_{n}=\{l L, l H\}$ and $K_{n}=\{l L, l H, h L\}$ cannot happen for the same parameter values.

The optimal sequential procedure has two potential advantages over the efficient auction. First, it can distort production. Second, it can distort the probabilities of winning. For example, a first 
period offer that is only acceptable to suppliers $l L$ and $l H$ distorts the probabilities that $l H$ wins, $x_{l H}$, upwards and distorts $x_{h L}$ downwards relative to the probabilities in the efficient auction, as is sometimes required in the optimal procedure. However, this comes at the cost of a distortion in the probabilities of allocating the contract to types $l L$ and $h H$. Indeed, it is easy to check that $x_{l L}<x_{l L}^{F B}$, unless the optimal offer in all rounds but the last is only acceptable to type $l L$ (and recall from Theorem 1 that $x_{l L}=x_{l L}^{F B}$ always in the optimal procedure). In addition, $x_{h H}>x_{h H}^{F B}$ in all cases except if $K_{n}=\{l L, l H, h L\}$ for all $n>1$.

These costs and benefits of the optimal sequential procedure are best illustrated for the case of two suppliers. To do this, we rewrite the expected utility from the efficient auction as $\sum p_{k} V W_{k}$ where $p_{k}$ is the probability that the mechanism allocates the contract to a type $k$ supplier and $V W_{k}$ is the associated virtual welfare. The next table summarizes the values that these variables take in the efficient auction (using Lemma 3):

Table 5: Virtual welfares and probabilities in the efficient auction

\begin{tabular}{|l|l|l|}
\hline \multicolumn{2}{|l|}{ Virtual welfares } & Probabilities \\
\hline$\Delta \theta_{1}-\Delta \theta_{2} \bar{q}>0$ & $\Delta \theta_{1}-\Delta \theta_{2} \bar{q}<0$ & $p_{k}=N \alpha_{k} x_{k}^{F B}$ \\
\hline$W_{l L}^{F B}$ & $W_{l L}^{F B}$ & $1-\left(1-\alpha_{l L}\right)^{2}$ \\
$W_{h L}^{F B}-\frac{\alpha_{l L}}{\alpha_{h L}} \Delta \theta_{1}$ & $W_{h L}^{F B}-\frac{\alpha_{l L}}{\alpha_{h L}} \Delta \theta_{1}$ & $\left(1-\alpha_{l L}\right)^{2}-\left(1-\alpha_{l L}-\alpha_{h L}\right)^{2}$ \\
$W_{l H}^{F B}$ & $W_{l H}^{F B}+\frac{\alpha_{l L}+\alpha_{h L}}{\alpha_{l H}} \Delta \theta_{1}-\frac{\alpha_{l L}+\alpha_{h L}}{\alpha_{l H}} \Delta \theta_{2} \bar{q}$ & $\left(1-\alpha_{l L}-\alpha_{h L}\right)^{2}-\left(1-\alpha_{l L}-\alpha_{h L}-\alpha_{l H}\right)^{2}$ \\
$W_{B}^{F B}-\frac{\alpha_{l H}}{\alpha_{h H}} \Delta \theta_{1}-\frac{\alpha_{h L}+\alpha_{l L}}{\alpha_{h H}} \bar{q}$ & $W_{h H}^{F B}-\frac{\alpha_{l H}+\alpha_{h L}+\alpha_{l L}}{\alpha_{h H}} \Delta \theta_{1}$ & $\alpha_{h H}^{2}$ \\
\hline
\end{tabular}

Similarly, the expected utility from the optimal sequential procedure can be written as $\sum \widetilde{p}_{k} \widetilde{V W}_{k}$ where $\widetilde{p}_{k}$ is the probability that the optimal sequential mechanism allocates the contract to supplier $k$ and $\widetilde{V W}_{k}$ is the "resulting" virtual welfare. ${ }^{10}$ The idea then is to compare the $p_{k}$ 's and $\widetilde{p}_{k}$ 's and the $V W_{k}$ 's with the $\widetilde{V W}_{k}$ 's. The first example illustrates the advantage of being able to distort qualities.

\section{Example 1: The optimal sequential procedure always does better than the efficient auction when $\Delta \theta_{1}$ is sufficiently small.}

When $\Delta \theta_{1}$ is small, the main source of adverse selection is marginal cost and suppliers $l L$ and $h L$, and $l H$ and $h H$ respectively, are very much alike. Consider the strategy that consists in making an offer that is only acceptable to suppliers $l L$ and $h L$ in the first period. Using

\footnotetext{
${ }^{10}$ We write "resulting" because the virtual welfare associated with a given type is not uniquely pinned down in this sequential mechanism. We exploit this flexibility in the remaining discussion.
} 
Lemma 6 and Theorem 3, the resulting expected utility is given by:

$$
\begin{aligned}
V_{2}= & \left(\alpha_{h L}+\alpha_{l L}\right) W_{h L}^{F B}+\left(\alpha_{l H}+\alpha_{h H}\right) V_{1} \\
= & \alpha_{l L}\left(2-\alpha_{h L}-\alpha_{l L}\right) W_{l L}^{F B} \\
& +\alpha_{h L}\left(2-\alpha_{h L}-\alpha_{l L}\right)\left(W_{h L}^{F B}-\frac{\alpha_{l L}}{\alpha_{h L}} \Delta \theta_{1}\right) \\
& +\left(\alpha_{l H}+\alpha_{h H}\right)^{2}\left(W_{h H}\left(q_{1}^{1}\right)-\frac{\left(\alpha_{h L}+\alpha_{l L}\right)}{\left(\alpha_{l H}+\alpha_{h H}\right)} \Delta \theta_{2} q_{1}^{1}\right)
\end{aligned}
$$

where we have grouped types $l H$ and $h H$. Comparing this expression with the second column of Table 5 suggests that $V W_{l L}=\widetilde{V W}_{l L}$ and $V W_{h L}=\widetilde{V W}_{h L}$. Moreover, $p_{h L}+p_{l L}=1-$ $\left(1-\alpha_{h L}-\alpha_{l L}\right)^{2}=\widetilde{p}_{h L}+\widetilde{p}_{l L}=\left(\alpha_{h L}+\alpha_{l L}\right)\left(2-\alpha_{h L}-\alpha_{l L}\right)$. Thus, when $\Delta \theta_{1}$ is very small, $p_{l L} V W_{l L}+p_{h L} V W_{h L} \simeq \widetilde{p}_{l L} \widetilde{V W}_{l L}+\widetilde{p}_{h L} \widetilde{V W}_{h L}$ since $V W_{h L} \simeq V W_{l L}$. Turning to the utility contribution of types $l H$ and $h H$ in the efficient auction, we get, using Table 5 and after some simplifications

$$
\begin{aligned}
& \alpha_{l H}\left(\alpha_{l H}+2 \alpha_{h H}\right) W_{l H}^{F B}+\alpha_{h H}^{2} W_{h H}^{F B}-\left(\alpha_{l H}+2 \alpha_{h H}\right)\left(\alpha_{h L}+\alpha_{l L}\right) \Delta \theta_{2} \bar{q} \\
& +\left(\left(\alpha_{l H}+\alpha_{h H}\right)\left(\alpha_{h L}+\alpha_{l L}\right)-\alpha_{l H} \alpha_{h H}\right) \Delta \theta_{1} \\
= & \left(\alpha_{l H}+\alpha_{h H}\right)^{2}\left[W_{h H}^{F B}-\frac{\left(\alpha_{l H}+2 \alpha_{h H}\right)\left(\alpha_{h L}+\alpha_{l L}\right)}{\left(\alpha_{l H}+\alpha_{h H}\right)^{2}} \Delta \theta_{2} \bar{q}\right] \\
& +\left(\alpha_{l H}+\alpha_{h H}\right)\left(1-\alpha_{h H}\right) \Delta \theta_{1}
\end{aligned}
$$

The first term of this expression is strictly less than $\left(\alpha_{l H}+\alpha_{h H}\right)^{2}\left(W_{h H}\left(q_{1}^{1}\right)-\frac{\alpha_{h L}+\alpha_{l L}}{\alpha_{l H}+\alpha_{h H}} \Delta \theta_{2} q_{1}^{1}\right)$ given the way $q_{1}^{1}$ is constructed (optimal level of distortion) and the fact that $\frac{\left(\alpha_{l H}+2 \alpha_{h H}\right)\left(\alpha_{h L}+\alpha_{l L}\right)}{\left(\alpha_{l H}+\alpha_{h H}\right)^{2}}>$ $\frac{\alpha_{h L}+\alpha_{l L}}{\alpha_{l H}+\alpha_{h H}}$. The second term becomes negligible as $\Delta \theta_{1}$ decreases. Thus for $\Delta \theta_{1}$ small enough, the optimal sequential procedure dominates the efficient auction because it is able to distort qualities.

Another way to view example 1 is to note that as $\Delta \theta_{1}$ converges to zero, the environment converges essentially to the "standard" one-dimensional environment, where the optimal procedure is such that $l L$ and $h L$ win over $h H$ and $l H$ and qualities are distorted. The sequential procedure replicates these features when $K_{2}=\{l L, h L\}$. In fact, the expected utility from the optimal sequential procedures converges to the expected utility from the optimal mechanism as $\Delta \theta_{1}$ decreases (see Table 6 below for numerical evidence on this).

The next example illustrates the advantage provided by the ability to distort allocation probabilities:

Example 2: For large values of $\Delta \theta_{1}$, the optimal sequential procedure can do better than the efficient auction. Consider the period 1 strategy that offers a contract to types 
$l L$ and $l H$ only. The resulting expected utility for the buyer is given by:

$$
\begin{aligned}
V_{2}= & \alpha_{l L}\left(W_{l L}^{F B}-\Delta \theta_{2} q_{1}^{*}\right)+\alpha_{l H} W_{l H}\left(q_{1}^{*}\right)+\left(1-\alpha_{l H}-\alpha_{l L}\right) V_{1} \\
= & \alpha_{l L}\left(2-\alpha_{l H}-\alpha_{l L}\right) W_{l L}^{F B} \\
& +\alpha_{h L}\left(1-\alpha_{l H}-\alpha_{l L}\right)\left(W_{h L}^{F B}-\frac{\alpha_{l L}}{\alpha_{h L}} \Delta \theta_{1}\right) \\
& +\alpha_{l H}\left(2-\alpha_{l H}-\alpha_{l L}\right)\left(\frac{1}{\left(2-\alpha_{l H}-\alpha_{l L}\right)} W_{l H}\left(q_{1}^{*}\right)+\frac{\left(1-\alpha_{l H}-\alpha_{l L}\right)}{\left(2-\alpha_{l H}-\alpha_{l L}\right)} W_{l H}\left(q_{1}^{1}\right)\right) \\
& +\alpha_{h H}\left(1-\alpha_{l H}-\alpha_{l L}\right)\left(W_{h H}\left(q_{1}^{1}\right)-\frac{\alpha_{l H}}{\alpha_{h H}} \Delta \theta_{1}-\frac{\left(\alpha_{h L}+\alpha_{l L}\right)}{\alpha_{h H}} \Delta \theta_{2} q_{1}^{1}-\frac{\alpha_{l L}}{\alpha_{h H}\left(\alpha_{h H}+\alpha_{h L}\right)} \Delta \theta_{2} q_{1}^{*}\right) \\
= & \sum_{k} \widetilde{p}_{k} \widetilde{V W}_{k}
\end{aligned}
$$

Comparing this with the probabilities and the levels of virtual welfare in Table 5, it is clear that $V W_{l L}=\widetilde{V W}_{l L}, V W_{h L}=\widetilde{V W}_{h L}, V W_{l H}>\widetilde{V W}_{l H}$ and $V W_{h H} \lessgtr \widetilde{V W}_{h H}$. Moreover, the sequential procedure essentially places $l H$ in front of $h L$ in the order of priority in the allocation, resulting in the following ordering of probabilities: $p_{l L}>\widetilde{p}_{l L}, p_{h L}>>\widetilde{p}_{h L}, p_{l H}<<$ $\widetilde{p}_{l H}$ and $p_{h H}<\widetilde{p}_{h H}$. When $\frac{\alpha_{l L}}{\alpha_{h L}} \Delta \theta_{1}$ is large enough, $V W_{h L}<<V W_{l H}, \widetilde{V W}_{l H}$. Thus, this allocation can increase expected utility.

Figure 7 illustrates an environment when both of these scenarios occur.

\section{Insert Figure 7 Here}

\subsection{Computational results}

The previous analysis has identified conditions in the environment that favor sequential mechanisms over the efficient auction. Another question concerns the relative performance of sequential mechanisms across a wide range of environments. Table 6 reports the proportion of the difference between the expected utility in the optimal mechanism and the expected utility from the efficient auction (i.e. the surplus available from being strategic) that the optimal sequential procedure captures. In the table, $v(q)$ takes the form $a q^{b}$, where $a=3$ and $b=0.5$. In all experiments, $l=L=1$, $H=2$ and $N=2$. Negative values indicate that the optimal sequential mechanism does worse than the efficient auction.

\section{Insert Table 6 Here}

The results are the following. On average (i.e. across all possible values of $\Delta \theta_{1}$ ), the optimal sequential procedure does worse, and often much worse, than the efficient auction. The poor performance of sequential mechanisms is confirmed by the small fraction of values for $\Delta \theta_{1}$ where 
the optimal sequential mechanism captures at least $80 \%$ of the surplus (second to last column) and where it does better than the efficient auction (last column).

There are two exceptions to the poor performance of sequential procedures. First, and as suggested by example 1, the optimal sequential procedure does very well and in fact as well as the optimal mechanism when $\Delta \theta_{1}=0$ (fourth column in the table).

Second, the optimal sequential mechanism does better overall when there is strong positive correlation between types (experiment 4 in the table). The reason is related to example 2 above: when costs are highly correlated, $\frac{\alpha_{l L}}{\alpha_{h L}}$ is high, and the virtual welfare associated with type $h L$ tends to be lower than the virtual welfare associated with $l H$. Thus, a contract only acceptable to types $l H$ and $l L$ in the first period reverses the order of priority of types $h L$ and $l H$ and can increase expected utility. Note, however, that this is not the end of the story. Indeed, the optimal sequential procedure does poorly in experiments 17 through 19, even though the ratio $\frac{\alpha_{l L}}{\alpha_{h L}}$ is high there too. The reason is that a first period offer acceptable to types $l L$ and $l H$ also increases the probability that a type $l H$ wins over a type $l L$. Experiments 17 through 19 illustrate that this is particularly costly in terms of expected utility when $\alpha_{l H}>\alpha_{l L}$.

\section{Concluding remarks}

In this paper, we have asked how a buyer should optimally structure its buying process when suppliers' private information is multidimensional and quality is contractable, and how well commonly used procedures such as scoring auctions and sequential procedures perform. We have answered the second question by combining a theoretical analysis of the restrictions that such simpler procedures impose on allocations, with numerical analyses of their performance relative to the benchmark of the optimal buying mechanism.

Our main results are that scoring auctions do well and that sequential procedures do badly, both relative to the optimal mechanism and relative to the efficient auction. In each case, we have identified the underlying reason and environments which represent exceptions to this general result. Our interpretation of both sets of results is that utility maximization is more about "getting allocation probabilities right" than distorting qualities. This is the main reason why scoring auctions do well and sequential procedures do so poorly. Because the scoring auction's "right kind of flexibility in terms of allocation probabilities" and the generic misallocation of contracts in sequential procedures are intrinsic features of these procedures and do not depend on the number of bidders, we are confident that the bottom line of our numerical results extends to more than two suppliers (Of course, as the number of suppliers goes to infinity it is straightforward to show that the expected utility from all procedures converge to the same value which is $W_{D}^{F B}$ - full extraction). From a practical perspective, our advice to professional buyers is: use a scoring auction, manipulate it slightly if you can commit, else just announce your true preferences regarding the price - quality 
trade-off.

An a priori restrictive assumption in our analysis is the binary structure of private information and it is worthwhile to comment on it here. First, we note that the main results concerning the optimal procedure (like the fact that it depends on the number of bidders, the fact that it involves both productive and allocative inefficiencies, and the fact that suppliers with the same marginal cost for quality generically supply different quality levels) are all driven by the endogeneity of the binding incentive compatibility constraints. For this reason, we expect them to hold in more general environments. Second, the generic misallocation of the sequential procedure can only become worse in richer informational environments, whereas scoring auctions continue to allocate the contract efficiently, conditional on the announced scoring rule. Thus, if our interpretation according to which getting the allocation right is the first order effect in procurement, then the dominance of scoring auctions is likely to extend. 


\section{References}

[1] Arizona Department of Transport (2002), A+B Bidding Guide, http://www.dot.state.az.us/roads/constgrp/A+BGuide.pdf (dated 1/18/2002).

[2] Armstrong, Mark (1996), Multiproduct Non-linear Pricing, Econometrica, 64, 51-74.

[3] Armstrong, Mark (2000), Optimal Multi-Object Auctions, Review of Economic Studies, 67, 455-481.

[4] Asker, John and Estelle Cantillon (2006), Properties of Scoring Auctions, NYU and ECARES mimeo

[5] Asker, John and Estelle Cantillon (2005), Optimal Procurement when both Price and Quality Matter, CEPR Discussion Paper 5276.

[6] Avery, Chris and T. Henderschott (2000), Bundling and Optimal Auctions of Multiple Objects, Review of Economic Studies, 67(3), 483-497.

[7] Bajari, Patrick, Robert McMillan and Steve Tadelis (2004), Auctions vs. Negotiation in Procurement: An Empirical Analysis, mimeo.

[8] Bajari, Patrick and Steve Tadelis (2001), Incentives Versus Transaction Costs: A Theory of Procurement Contracts, Rand Journal of Economics, 32(3), 287-307

[9] Border, Kim (1991), Implementation of Reduced Form Auctions: A Geometric Approach, Econometrica, 59(4), 1175-1187.

[10] Branco, Fernando (1997), The Design of Multidimensional Auctions, Rand Journal of Economics, 28(1), Spring 1997, pp. 63-81

[11] Bulow, Jeremy and Paul Klemperer (1996), Auctions versus Negotiation, American Economic Review, 86(1), 180-194.

[12] Che, Yeon-Koo (1993), Design Competition through Multidimensional Auctions, Rand Journal of Economics, 668-680.

[13] Che, Yeon-Koo and Ian Gale (2003), Optimal Design of Research Contests, American Economic Review, 93,

[14] Che, Yeon-Koo (2006), Procurement, forthcoming The New Palgrave. Dictionary of Economics (S. Durlauf and L. Blume, Eds), Second Edition.

[15] Chen-Ritzo, Harrison, Kwasnica and Thomas (2003), Better, Faster, Cheaper: a multiattribute supply chain auction mechanism, Penn State mimeo. 
[16] Dasgupta, S. and D. Spulber (1989), Managing Procurement Auctions, Information Economics and Policy, 4, 5-29.

[17] De Frutos, Maria-Angeles and Lambros Pechlivanos (2004), Auctioning Off with a Split Mind: Privatization under Political Constraints, Carlos III mimeo.

[18] Ganuza, Juan-Jose and Lambros Pechlivanos (2000), Heterogeneity Promoting Procurement, Economics Letters, 67(1), 105-112.

[19] Herbsman, Zohar, Wei Tong Chen and William C. Epstein (1995), Time is Money: Innovative Contracting Methods in Highway Construction, Journal of Construction Engineering and Management, 121(3), 273-281.

[20] Jehiel, Philippe, Benny Moldovanu and Ennio Stachetti (1999), Multi-dimensional Mechanism Design for Auctions with Externalities, Journal of Economic Theory, 85(2), 258-94.

[21] Klein, B. and K.B. Leffler (1981), The Role of Market Forces in Assuring Contractual Performance, Journal of Political Economy, 89, 615-41.

[22] Laffont, Jean-Jacques and Jean Tirole (1987), Auctioning Incentive Contracts, Journal of Political Economy, 95(5), 921-937.

[23] Malakhov, Alexey and Rakesh Vohra (2004), Single and Multidimensional Optimal Auctions - A Network Approach, MEDS mimeo.

[24] Manelli, Alejandro and Daniel Vincent (1995), Optimal Procurement Mechanisms, Econometrica, 63(3), 591-620.

[25] Manelli, Alejandro and Daniel Vincent (2004), Multidimensional Mechanism Design: Revenue Maximization and the Multiple-Good Monopoly, Arizona State mimeo.

[26] Morand, Pierre-Henri and Lionel Thomas (2002), Optimal Procurement Rules with Asymmetries on Quality and Efficiency, CRESE mimeo.

[27] Rezende, Leonardo (2003), Biased Procurement, Stanford mimeo.

[28] Rochet, Jean-Charles and Philippe Choné (1998), Ironing, Sweeping, and Multidimensional Screening, Econometrica, 66(4), 783-826.

[29] Rochet, Jean-Charles and Lars Stole (2003), The Economics of Multidimensional Screening, in: Dewatripont, Hansen and Turnovsky (eds), Advances in Economics and Econometrics: Theory and Applications, Eighth World Congress, Cambridge University Press.

[30] Taylor, C. (1993), Delivery-Contingent Contracts for Research, Journal of Law, Economics and Organization, 9, 188-203. 
[31] Wang, G.H. (1998), Bargaining over a Menu of Wage Contracts, Review of Economic Studies, 65, 295-305.

[32] Wilson, Robert (1993), Nonlinear Pricing, Oxford University Press. 


\section{Appendix}

Lemma 1: Consider the feasibility constraints

$$
N \sum_{k \in K} \alpha_{k} x_{k} \leq 1-\left(1-\sum_{k \in K} \alpha_{k}\right)^{N} \text { for all subsets } K \text { of }\{h H, l H, h L, l L\}
$$

and define an n-type constraint as a feasibility constraint with the relevant subset $K$ having $n$ elements. The following statements hold:

i. At most one one-type constraint binds, at most one two-type constraint binds and at most one three-type constraint binds.

ii. These binding constraints are nested, in the sense that the type in the binding one-type constraint must belong to the binding two-type constraint, and so on.

Proof of Lemma 1: The claim relies on the fact that the function $f(t)=t^{N}$ for $N \geq 2$ is strictly convex. There are two generic cases to rule out: two constraints binding with no type in common, and two non nested constraints binding with some type in common.

Case 1: No overlap. Suppose, towards a contradiction, that the constraint for $l H$ and the constraint for $\{h H, h L\}$ bind. Then, from (4), $N\left(\alpha_{l H} x_{l H}+\alpha_{h H} x_{h H}+\alpha_{h L} x_{h L}\right)=2-\left(1-\alpha_{l H}\right)^{N}-\left(1-\alpha_{h H}-\right.$ $\left.\alpha_{h L}\right)^{N}>1-\left(1-\alpha_{l H}-\alpha_{h H}-\alpha_{h L}\right)^{N}$ since $1+\left(1-\alpha_{l H}-\alpha_{h H}-\alpha_{h L}\right)=\left(1-\alpha_{l H}\right)+\left(1-\alpha_{h H}-\alpha_{h L}\right)$ and $\left(1-\alpha_{l H}\right)$ and $\left(1-\alpha_{h H}-\alpha_{h L}\right)$ lie in $\left(1-\alpha_{l H}-\alpha_{h H}-\alpha_{h L}, 1\right)$. That is, (4) is violated for $\{l H, h H, h L\}$. All cases with no overlap are proved in this way.

Case 2: Some overlap. Suppose, towards a contradiction that the constraint for $\{l H, h H\}$, and that for $\{h H, h L\}$ is binding. Since (4) holds for $h H$, this means that

$$
\begin{aligned}
N\left(\alpha_{l H} x_{l H}+\alpha_{h H} x_{h H}+\alpha_{h L} x_{h L}\right) & \geq 1-\left(1-\alpha_{l H}-\alpha_{h H}\right)^{N}-\left(1-\alpha_{h H}-\alpha_{h L}\right)^{N}+\left(1-\alpha_{h H}\right)^{N} \\
& >1-\left(1-\alpha_{l H}-\alpha_{h H}-\alpha_{h L}\right)^{N} \text { by convexity }
\end{aligned}
$$

This contradicts (4) for $\{l H, h H, h L\}$. All cases with some overlap are proved in this way.

This proves that binding constraints are nested and that they cannot be more than one constraint of a type to bind. Q.E.D.

Lemma 3 (Binding constraints in the efficient auction): When $\Delta \theta_{1}>\Delta \theta_{2} \bar{q}, I C_{l H, h H}$, $I C_{h L, h H}$ and $I C_{l L, h L}$ bind in the buyer-optimal efficient mechanism. When $\Delta \theta_{1}<\Delta \theta_{2} \bar{q} I C_{l H, h H}$, $I C_{h L, h H}$ and $I C_{l L, h L}$ bind.

Proof of Lemma 3: Let $U_{k, j}$ be the expected utility of a type $k$ pretending she is of type $j$. To satisfy incentive compatibility, while minimizing suppliers' rents, suppliers' expected utilities must be set such that $U_{k}=\max _{j \neq k} U_{k, j}$. Let $U_{h H}=0$ (we can check ex post that this will satisfy supplier $h H$ 's incentive compatibility constraints).

Given the parameters of the model, we first argue that $U_{h L}=\max \left\{U_{h L, h H}, U_{h L, l H}\right\}$, i.e., that imitating $l L$ is dominated for supplier $h L$. Indeed, $U_{h L, l L}=U_{l L}-x_{l L}^{F B} \Delta \theta_{1}$. If $U_{l L}=U_{l L, h L}$, we get $U_{h L, l L}=U_{h L}+\left(x_{h L}^{F B}-x_{l L}^{F B}\right) \Delta \theta_{1}<U_{h L}$. If instead, $U_{l L}=U_{l L, l H}$, we have $U_{h L, l L}=U_{l H}+$ 
$x_{l H} \Delta \theta_{2} \bar{q}-x_{l L}^{F B} \Delta \theta_{1}<U_{l H}+x_{l H}^{F B} \Delta \theta_{2} \bar{q}-x_{l H}^{F B} \Delta \theta_{1}=U_{h L, l H} \leq U_{h L}$ (we can rule out $U_{l L}=U_{l L, h H}$ since it is dominated for supplier $l L)$.

Second, we argue that $U_{l H}=U_{l H, h H}$. The first alternative for supplier $l H$ is that he imitates $h L$. Her expected payoff in this case is $U_{l H, h L}=U_{h L}+x_{h L}^{F B} \Delta \theta_{1}-x_{h L}^{F B} \underline{q} \Delta \theta_{2}$ where $U_{h L}=$ $\max \left\{U_{h L, h H}, U_{h L, l H}\right\}$. We consider each case in turn. (a) If $U_{h L}=U_{h L, h H}$, the requirement of $U_{l H, h L}<U_{l H, h H}$ is equivalent to $x_{h L}^{F B}\left(\Delta \theta_{1}-\Delta \theta_{2} \underline{q}\right)-x_{h H}^{F B}\left(\Delta \theta_{1}-\Delta \theta_{2} \bar{q}\right)<0$. The first term is negative since, by assumption, $W_{l H}(\bar{q})<W_{h L}(\underline{q})$. The second term may be positive or negative, but even when it is negative, $x_{h L}^{F B}\left(\Delta \theta_{1}-\Delta \theta_{2} \underline{q}\right)<x_{h H}^{F B}\left(\Delta \theta_{1}-\Delta \theta_{2} \bar{q}\right)<0$ since $\underline{q}>\bar{q}$ and $x_{h H}^{F b}<x_{h L}^{F B}$. (b) If $U_{h L}=U_{h L, l H}, U_{l H, h L}<U_{l H, h H}$ is equivalent to $x_{h L}^{F B}\left(\Delta \theta_{1}-\Delta \theta_{2} q\right)-x_{l H}^{F B}\left(\Delta \theta_{1}-\Delta \theta_{2} \bar{q}\right)<0$. This holds by exactly the same reasoning.

The second alternative is $U_{l H, l L}=U_{l L}-x_{l L}^{F B} \Delta \theta_{2} q$. When $U_{l L}=U_{l L, l H}, U_{l H, l L}=U_{l H}+x_{l H}^{F B} \Delta \theta_{2} \bar{q}-$ $x_{l L}^{F B} \Delta \theta_{2} \underline{q}<U_{l H}$. When $U_{l L}=U_{l L, h L}, U_{l H, l L}=U_{h L}+x_{h L}^{F B} \Delta \theta_{1}-x_{l L}^{F B} \Delta \theta_{2} \underline{q}<U_{l H, h L}=U_{h L}+$ $x_{h L}^{F B} \Delta \theta_{1}-x_{h L}^{F B} \Delta \theta_{2} \underline{q}$. We conclude that $U_{l H}=U_{l H, h H}$.

Finally, we show that $U_{l L}=U_{l L, h L}$. When $U_{h L}=U_{h L, l H}, U_{l L, h L}=x_{h L}^{F B} \Delta \theta_{1}-x_{l H}^{F B} \Delta \theta_{1}+x_{l H}^{F B} \bar{q} \Delta \theta_{2}+$ $x_{h H}^{F B} \Delta \theta_{1}>U_{l L, l H}=x_{l H}^{F B} \bar{q} \Delta \theta_{2}+x_{h H}^{F B} \Delta \theta_{1}$ since $x_{h L}^{F B}>x_{l H}^{F B}$. When $U_{h L}=U_{h L, h H}$, the claim follows from the fact that $x_{h L}^{F B} \Delta \theta_{1}-x_{l H}^{F B} \Delta \theta_{2} \bar{q}>x_{l H}^{F B}\left(\Delta \theta_{1}-\Delta \theta_{2} \bar{q}\right)>x_{h H}^{F B}\left(\Delta \theta_{1}-\Delta \theta_{2} \bar{q}\right)$ (the last inequality being implied by $\left.U_{h L, h H}>U_{h L, l H}\right)$. This leads us to:

$$
\begin{aligned}
U_{l H} & =U_{l H, h H}=x_{h H}^{F B} \Delta \theta_{1} \\
U_{h H} & =0 \\
U_{h L} & =\max \left\{U_{h L, h H}, U_{h L, l H}\right\}=\max \left\{x_{h H}^{F B} \bar{q} \Delta \theta_{2},-x_{l H}^{F B}\left(\Delta \theta_{1}-\Delta_{2} \bar{q}\right)+x_{h H}^{F B} \Delta \theta_{1}\right\} \\
U_{l L} & =U_{l L, h L}=x_{h L}^{F B} \Delta \theta_{1}+U_{h L}
\end{aligned}
$$

In practice, this generates two cases depending on the sign of $\Delta \theta_{1}-\Delta_{2} \bar{q}$. When $\Delta \theta_{1}-\Delta_{2} \bar{q}>0$, $U_{h L, h H}>U_{h L, l H}$. When $\Delta \theta_{1}-\Delta_{2} \bar{q}<0, U_{h L, l H}>U_{h L, h H}$. Q.E.D.

Lemma 4: The first order conditions of the maximization problem (1) subject to (2), (3), (4) and (5) are necessary and sufficient for a global maximum.

Proof of Lemma 4: Consider the following change of variables: $z_{1 k}=x_{k}, z_{2 k}=x_{k} q_{k}$. Let $\widetilde{F}\left(z_{1 k}, z_{2 k}, U_{k}\right)=N \sum_{k=l H, h H, h L, l L} \alpha_{k}\left(z_{1 k} W_{k}\left(\frac{z_{2 k}}{z_{1 k}}\right)-U_{k}\right)$. The problem becomes:

$$
\begin{aligned}
& \max _{z_{1 k}, z_{2 k}, U_{k}} \tilde{F}\left(z_{1 k}, z_{2 k}, U_{k}\right) \quad \text { s.t. } \\
U_{k} & \geq U_{j}+z_{1 j}\left(\theta_{1 j}-\theta_{1 k}\right)+z_{2 j}\left(\theta_{2 j}-\theta_{2 k}\right) \quad \text { for all } k, j \in\{h H, l H, h L, l L\} \\
U_{k} & \geq 0 \quad \text { for all } k \in\{h H, l H, h L, l L\} \\
N \sum_{k \in K} \alpha_{k} z_{1 k} & \leq 1-\left(1-\sum_{k \in K} \alpha_{k}\right)^{N} \text { for all subsets } K \text { of }\{h H, l H, h L, l L\} \\
N \sum_{k \in\{h H, l H, h L, l L\}} \alpha_{k} z_{1 k} & =1
\end{aligned}
$$


The constraints are linear in the control variables so the constraint qualification holds and the objective function is concave. ${ }^{11}$ The first order conditions are thus necessary and sufficient for a global maximum. To prove that the first order conditions of the original problem are also necessary and sufficient, we need to check that the first order conditions of the two problems are equivalent. To see this, let $G\left(x_{k}, q_{k}, U_{k}\right)$ gather all constraint terms of the Lagrangian of the original problem, and $\widetilde{G}\left(z_{1 k}, z_{2 k}, U_{k}\right)$ gather the constraint terms of the Lagrangian of the transformed problem. We must show that $\left(x_{k}^{*}, q_{k}^{*}, U_{k}^{*}\right)$ solves the first order conditions of $\max _{x_{k}, q_{k}, U_{k}} F\left(x_{k}, q_{k}, U_{k}\right)+G\left(x_{k}, q_{k}, U_{k}\right)$ if and only if $\left(x_{k}^{*}, x_{k}^{*} q_{k}^{*}, U_{k}^{*}\right)$ solves the first order conditions of $\max _{z_{1 k}, z_{2 k}, U_{k}} \widetilde{F}\left(z_{1 k}, z_{2 k}, U_{k}\right)+\widetilde{G}\left(z_{1 k}, z_{2 k}, U_{k}\right)$. The first order conditions with respect to $U_{k}$ are identical. The first order condition with respect to $q_{k}, F_{q_{k}}\left(x_{k}^{*}, q_{k}^{*}, U_{k}^{*}\right)+G_{q_{k}}\left(x_{k}^{*}, q_{k}^{*}, U_{k}^{*}\right)=0$, takes the form

$$
N \alpha_{k} x_{k}^{*} W_{k}^{\prime}\left(q_{k}^{*}\right)-\sum \lambda_{l} x_{k}^{*}\left(\theta_{2 k}-\theta_{2 l}\right)=0
$$

(where $\lambda_{l}$ are the Lagrangian multipliers of the constraints). This is equivalent to the first order conditions of the transformed problem with respect to $z_{2 k}$,

$$
N \alpha_{k} W_{k}^{\prime}\left(\frac{z_{2 k}}{z_{1 k}}\right)-\sum \lambda_{l}\left(\theta_{2 k}-\theta_{2 l}\right)=0
$$

as long as $x_{k}^{*}>0$ for all $k$, a consequence of the non exclusion condition (5). Finally, the first order condition with respect to $x_{k}, F_{x_{k}}\left(x_{k}^{*}, q_{k}^{*}, U_{k}^{*}\right)+G_{x_{k}}\left(x_{k}^{*}, q_{k}^{*}, U_{k}^{*}\right)=0$ takes the form:

$$
N \alpha_{k} W_{k}\left(q_{k}^{*}\right)-\sum \lambda_{l}\left[\left(\theta_{1 k}-\theta_{1 l}\right)+q_{k}^{*}\left(\theta_{2 j}-\theta_{2 l}\right)\right]-N \sum_{K \text { st } k \in K} \gamma_{K} \alpha_{k}=0
$$

The first order condition of the transformed problem takes the form:

$$
N \alpha_{k} W_{k}\left(\frac{z_{2 k}}{z_{1 k}}\right)-N \alpha_{k} \frac{z_{2 k}}{z_{1 k}} W_{k}^{\prime}\left(\frac{z_{2 k}}{z_{1 k}}\right)-\sum \lambda_{l}\left(\theta_{1 j}-\theta_{1 l}\right)-N \sum_{K \text { st } k \in K} \gamma_{K} \alpha_{k}=0
$$

This is equivalent to (9) as soon as (8) holds. QED

Theorem 2: An allocation can be implemented with a scoring auction if and only if (1) $q_{l H}=q_{h H}$, $q_{h L}=q_{l L}$ with $q_{l H}, q_{h H}<q_{h L}, q_{l L}$, (2) $\alpha_{l H} x_{l H}+\alpha_{h L} x_{h L}=\alpha_{l H} x_{l H}^{F B}+\alpha_{h L} x_{h L}^{F B}, x_{h H}=x_{h H}^{F B}$ and $x_{l L}=x_{l L}^{F B}$, (3) $\Delta \theta_{1}-\Delta \theta_{2} q_{h L} \leq 0$ when $x_{h L}>x_{h L}^{\min }$ and (4) $\Delta \theta_{1}-\Delta \theta_{2} q_{l H} \geq 0$ whenever the allocation is such that $x_{l H}>x_{l H}^{F B}$.

Proof of Theorem 2: Let $S_{k}(q)=\widetilde{v}(q)-\theta_{1 k}-\theta_{2 k} q$. We first prove the necessary conditions. Recall from the discussion in the main text that, in a scoring auction, suppliers select their offers to maximize the score they generate, given their profit target, $\left\{\widetilde{v}(q)-\theta_{1 i}-\theta_{2 i} q-\pi\right\}$. The solution only depends on suppliers' marginal cost, which establishes condition (1) given that $\theta_{2 l H}=\theta_{2 h H}>$

\footnotetext{
${ }^{11}$ The hessian is block diagonal with each block given by $\left[\begin{array}{ccc}\alpha_{k} \frac{z_{2 k}^{2}}{z_{1 k}^{3}} W^{\prime \prime} & -\alpha_{k} \frac{z_{2 k}}{z_{1 k}^{2}} W^{\prime \prime} & 0 \\ -\alpha_{k} \frac{z_{2 k}}{z_{1 k}^{2}} W^{\prime \prime} & \alpha_{k} \frac{W^{\prime \prime}}{z_{1 k}} & 0 \\ 0 & 0 & 0\end{array}\right]$
} 
$\theta_{2 h L}=\theta_{2 l L}$. Condition (2) follows from the fact that $l L$ can always generate a strictly higher score than either $l H$ and $h L$ for all choices of the scoring rule $\widetilde{v}($.$) . Similarly, both l H$ and $h L$ can always generate a strictly higher score than $h H$ so they must win against a $h H$ type.

When $x_{h L}>x_{h L}^{\min }, S_{h L}\left(q_{h L}\right) \geq S_{l H}\left(q_{l H}\right)$, else $l H$ should have priority over $h L$ in the allocation. This implies that

$$
\begin{aligned}
\widetilde{v}\left(q_{h L}\right)-h-L q_{h L} & \geq \widetilde{v}\left(q_{l H}\right)-l-H q_{l H}, \text { that is, } \\
\Delta \theta_{1}-\Delta \theta_{2} q_{h L} & \leq \widetilde{v}\left(q_{h L}\right)-\widetilde{v}\left(q_{l H}\right)-H\left(q_{h L}-q_{l H}\right)
\end{aligned}
$$

In addition, incentive compatibility requires that $l H$ generates a higher score by choosing $q_{l H}$ than $q_{h L}$, i.e.

$$
\widetilde{v}\left(q_{h L}\right)-\widetilde{v}\left(q_{l H}\right)-H\left(q_{h L}-q_{l H}\right) \leq 0
$$

Combining both inequalities yields condition (3). Similarly, when $x_{l H}>x_{l H}^{F B}, S_{l H}\left(q_{l H}\right) \geq S_{h L}\left(q_{h L}\right)$, else $h L$ should have priority in the allocation. This implies $\Delta \theta_{1}-\Delta \theta_{2} q_{l H}+L\left(q_{h L}-q_{l H}\right)+\widetilde{v}\left(q_{l H}\right)-$ $\widetilde{v}\left(q_{h L}\right) \geq 0$. In addition, $h L$ must be generating a higher score by choosing $q_{h L}$ over $q_{l H}$, i.e. $L\left(q_{h L}-q_{l H}\right)+\widetilde{v}\left(q_{l H}\right)-\widetilde{v}\left(q_{h L}\right) \leq 0$. Combining both inequalities yields condition (4).

To prove sufficiency, we construct a scoring rule that implements the intended allocation in a second score auction (in a second score auction, it is a dominant strategy to submit bids generating scores $\left.S_{k}\left(q_{k}\right)=\max _{q}\left\{\widetilde{v}(q)-\theta_{1 k}-\theta_{2 k} q\right\}\right)$. Consider

$$
\widetilde{v}(q)=v(q) 1_{\left\{q \leq q_{l H}\right\}}+v\left(q_{l H}\right) 1_{\left\{q>q_{l H}\right\}}+\epsilon 1_{\left\{q \geq q_{h L}\right\}}
$$

For this scoring auction to implement the outcome, two conditions must be satisfied. First, suppliers must be choosing the assigned qualities when they maximize their scores. Second, the ranking of the scores must (weakly) correspond to the assigned ranking of types in the allocation.

Given the shape of this scoring rule, the two relevant choices are $q_{l H}$ and $q_{h L} . l H$ prefers $q_{l H}$ to $q_{h L}$ if and only if $v\left(q_{l H}\right)-l-H q_{l H} \geq v\left(q_{l H}\right)+\varepsilon-l-H q_{h L}$ i.e. $\varepsilon \leq H\left(q_{h L}-q_{l H}\right)(h H$ 's preferences yield the same condition). $h L$ prefers $q_{h L}$ to $q_{l H}$ if and only if $v\left(q_{l H}\right)+\varepsilon-h-L q_{h L} \geq v\left(q_{l H}\right)-h-L q_{l H}$, i.e. $\varepsilon \geq L\left(q_{h L}-q_{l H}\right)$ (lL's preferences yield the same condition). Hence, suppliers choose their assigned qualities if $\varepsilon$ satisfies the following inequalities:

$$
L\left(q_{h L}-q_{l H}\right) \leq \varepsilon \leq H\left(q_{h L}-q_{l H}\right)
$$

which is possible by condition (1). Next, $h L$ generates a higher score if and only if $S_{h L}\left(q_{h L}\right)=$ $v\left(q_{l H}\right)+\varepsilon-h-L q_{h L} \geq S_{l H}\left(q_{l H}\right)=v\left(q_{l H}\right)-l-H q_{l H}$ i.e.

$$
\varepsilon \geq \Delta \theta_{1}-H q_{l H}+L q_{h L}=\Delta \theta_{1}-\Delta \theta_{2} q_{h L}+H\left(q_{h L}-q_{l H}\right)
$$

$l H$ generates a higher score otherwise. Inequalities (10) and (11) are always compatible if $\Delta \theta_{1}-$ $\Delta \theta_{2} q_{h L} \leq 0$ holds. When the solution is such that $x_{l H}>x_{l H}^{F B}$, we need $S_{l H}\left(q_{l H}\right) \geq S_{h L}\left(q_{h L}\right)$ :

$$
\varepsilon \leq \Delta \theta_{1}-H q_{l H}+L q_{h L}=\Delta \theta_{1}-\Delta \theta_{2} q_{l H}+L\left(q_{h L}-q_{l H}\right)
$$


instead. It is compatible with (10) if $\Delta \theta_{1}-\Delta \theta_{2} q_{l H} \geq 0$. Q.E.D.

Theorem 3 (characterization of the optimal sequential procedure): The optimal sequential procedure is such that the buyer offers the menu of contracts defined in Lemma 6 in the last round and, when $n>1$, one of the four following pairs of contracts, depending on which one yields the largest continuation value (conditional on the condition in the third column being satisfied):

\begin{tabular}{lll}
\hline$K_{n}$ & Offers $\left(p_{1}^{n}, q_{1}^{n}\right)$ and $\left(p_{2}^{n}, q_{2}^{n}\right)$ & $V_{n}$ \\
\hline \hline$l L$ & $\left(\underline{\theta}_{1}+\underline{\theta}_{2} \underline{q}, \underline{q}\right)$ & $\alpha_{l L} W_{l L}^{F B}+\left(1-\alpha_{l L}\right) V_{n-1}$ \\
\hline$l L, l H$ & $\left(\underline{\theta}_{1}+\bar{\theta}_{2} q_{1}^{*}, q_{1}^{*}\right)$ & $\alpha_{l L}\left(W_{l L}^{F B}-\Delta \theta_{2} q_{1}^{*}\right)+$ \\
& $\left(\underline{\theta}_{1}+\underline{\theta}_{2} \underline{q}+\Delta \theta_{2} q_{1}^{*}, \underline{q}\right)$ & $\alpha_{l H} W_{l H}\left(q_{1}^{*}\right)+\left(\alpha_{h L}+\alpha_{h H}\right) V_{n-1}$ \\
& $q_{1}^{*}=\arg \max \left\{v(q)-\bar{\theta}_{2} q-\frac{\alpha_{l L}}{\alpha_{l H}} \Delta \theta_{2} q\right\}$ & Condition: $\Delta \theta_{1}-\Delta \theta_{2} q_{1}^{*} \geq 0$ \\
\hline$l L, h L$ & $\left(\bar{\theta}_{1}+\underline{\theta}_{2} \underline{q}, \underline{q}\right)$ & $\left(\alpha_{l L}+\alpha_{h L}\right) W_{h L}^{F B}+\left(\alpha_{l H}+\alpha_{h H}\right) V_{n-1}$ \\
\hline$l L, l H, h L$ & $\left(\underline{\theta}_{1}+\bar{\theta}_{2} q_{1}^{* *}, q_{1}^{* *}\right)$ & $\left(\alpha_{l L}+\alpha_{h L}\right)\left(W_{l L}^{F B}-\Delta \theta_{2} q_{1}\right)$ \\
& $\left(\underline{\theta}_{1}+\underline{\theta}_{2} \underline{q}+\Delta \theta_{2} q_{1}^{* *}, \underline{q}\right)$ & $\alpha_{l H} W_{l H}\left(q_{1}^{* *}\right)+\alpha_{h H} V_{n-1}$ \\
& $q_{1}^{* *}=\arg \max \left\{v(q)-\bar{\theta}_{2} q-\frac{\left(\alpha_{l L}+\alpha_{h L}\right)}{\alpha_{l H}} \Delta \theta_{2} q\right\}$ & Condition: $\Delta \theta_{1}-\Delta \theta_{2} q_{1}^{* *} \leq 0$ \\
\hline
\end{tabular}

(The first column in the table indicates the set of supplier types who will accept the buyer's offer when $n$ suppliers remain, and the third column indicates the buyer's continuation value, $\left.V_{n}\right)$.

(Suppliers of types $l H$ and $h H$ accept the contract $\left(p_{1}^{n}, q_{1}^{n}\right)$ if it satisfies their IR constraint, and likewise, suppliers of types $l L$ and $h L$ accept contract $\left(p_{2}^{n}, q_{2}^{n}\right)$ if it satisfies their IR constraint).

Proof: Since suppliers are only made an offer once, they accept this offer whenever it contains a contract that satisfies their IR constraint. By design, the contracts will be such that $\left(p_{1}^{n}, q_{1}^{n}\right)$ (resp. $\left.\left(p_{2}^{n}, q_{2}^{n}\right)\right)$ is the contract chosen by the high (resp. low) marginal cost suppliers.

Let $K_{n}$ be the set of supplier types who accept one of the offers when $n$ suppliers remain. Given suppliers' cost structure, $K_{n} \in\{\{l L\},\{l L, l H\},\{l L, h L\},\{l L, l H, h L\},\{l H, h H, h L, l L\}\}$. We first argue that $K_{n} \neq\{l H, h H, h L, l L\}$, for $n>1$, i.e. some exclusion is optimal. By offering the single contract $\left(\underline{\theta}_{1}+\underline{\theta}_{2} \underline{q}, \underline{q}\right)$ that excludes all suppliers but $l L$, and the last round contract forever after (Lemma 6), the buyer guarantees himself an expected utility of $\alpha_{l L} W_{l L}^{F B}+\left(\alpha_{l H}+\alpha_{h H}+\alpha_{h L}\right) V_{1}$ $>V_{1}$, his continuation value if $K_{n}=\{l H, h H, h L, l L\}$. We now examine the optimal offers for the other three inclusion sets.

$K_{n}=\{l H, l L\}: l H$ 's IR constraint is binding, $p_{1}=\underline{\theta}_{1}+\bar{\theta}_{2} q_{1}$ and $l L$ 's IC constraint is binding, $p_{2}=p_{1}-\underline{\theta}_{2} q_{1}+\underline{\theta}_{2} q_{2}$. The optimal qualities solve $\alpha_{l H}\left(v\left(q_{1}\right)-\underline{\theta}_{1}-\bar{\theta}_{2} q_{1}\right)+\alpha_{l L}\left(v\left(q_{2}\right)-\underline{\theta}_{1}-L q_{2}-\Delta \theta_{2} q_{1}\right)$, thus $q_{1}^{*}=\arg \max \left\{v(q)-\bar{\theta}_{2} q-\frac{\alpha l L}{\alpha_{l H}} \Delta \theta_{2} q\right\}$. For this solution to be feasible we need in addition that $h L$ is indeed excluded, i.e. that $p_{1}-\bar{\theta}_{1}-\underline{\theta}_{2} q_{1}^{*} \leq 0$, i.e. $\Delta \theta_{1}-\Delta \theta_{2} q_{1}^{*} \geq 0$.

$\underline{K_{n}=\{h L, l L\}}$ : Only one contract is offered in this case: $\left(\bar{\theta}_{1}+\underline{\theta}_{2} \underline{q}, \underline{q}\right)$. For this solution to be feasible, $l H$ 's IR constraint must be violated, i.e. $\Delta \theta_{1}-\Delta \theta_{2} \underline{q} \leq 0$, which is automatically satisfied in our model. 
$K_{n}=\{l H, h L, l L\}:$ As before the binding IC constraint is from $l L$ and $h L$ to $l H$ (this will hold as long as $q_{1} \leq q_{2}$ ), hence $p_{2}-\underline{\theta}_{2} q_{2}=p_{1}-\underline{\theta}_{2} q_{1}$. We need to distinguish between two scenarios depending on which, from $l H$ or $h L$ 's IR constraints, is binding. Case 1: $l H$ 's IR constraint is binding at the optimum, i.e., $p_{1}=\underline{\theta}_{1}+\bar{\theta}_{2} q_{1}$. Substituting into the buyer's utility function, the resulting qualities solve $\alpha_{l H}\left(v\left(q_{1}\right)-\underline{\theta}_{1}-\bar{\theta}_{2} q_{1}\right)+\left(\alpha_{h L}+\alpha_{l L}\right)\left(v\left(q_{2}\right)-\underline{\theta}_{1}-\underline{\theta}_{2} q_{2}-\Delta \theta_{2} q_{1}\right)$. The last condition to check is that $h L$ 's IR constraint is indeed satisfied i.e. $\Delta \theta_{1}-\Delta \theta_{2} q_{1}^{* *} \leq 0$. Case 2 : $h L$ 's IR constraint is binding at the optimum, i.e. $p_{2}=\bar{\theta}_{1}+\underline{\theta}_{2} q_{2}$. Hence the resulting qualities must solve $\alpha_{l H}\left(v\left(q_{1}\right)-\bar{\theta}_{1}-\underline{\theta}_{2} q_{1}\right)+\left(\alpha_{h L}+\alpha_{l L}\right)\left(v\left(q_{2}\right)-\bar{\theta}_{1}-\underline{\theta}_{2} q_{2}\right)$, that is, $q_{1}=q_{2}=\underline{q}$ : the buyer offers a single contract. $l H$ 's IR constraint is satisfied if $\Delta \theta_{1}-\Delta \theta_{2} \underline{q} \geq 0$. (This is ruled out by assumption). Q.E.D. 
Table 1: Probabilities of winning and quality levels when $\Delta \theta_{1}-\Delta \theta_{2} \bar{q} \geq 0$

\begin{tabular}{|c|c|c|c|c|c|}
\hline Solution & Probabilities of Winning & $q_{l L}$ & $q_{h L}$ & $q_{l H}$ & $q_{h H}$ \\
\hline \multicolumn{6}{|c|}{ Condition: $x_{h H}^{F B}\left[\Delta \theta_{1}-\Delta \theta_{2} q_{h H}^{2}\right] \leq x_{l H}^{F B}\left[\Delta \theta_{1}-\Delta \theta_{2} \bar{q}\right]$} \\
\hline 1.1.a & $x_{k}=x_{k}^{F B}$ & $\underline{q}$ & $\underline{q}$ & $\bar{q}$ & $q_{h H}^{2}$ \\
\hline 1.1.b & $x_{l L}=x_{l L}^{F B}>x_{l H}=x_{l H}^{\max ^{\prime}}>x_{h L}=x_{h L}^{\min }>x_{h H}=x_{h H}^{F B}$ & $\underline{q}$ & $\underline{q}$ & $\bar{q}$ & $q_{h H}^{2}$ \\
\hline 1.1.c & $x_{l L}=x_{l L}^{F B}>x_{l H}^{\max } \geq x_{l H}>x_{h L} \geq x_{h L}^{\min }>x_{h H}=x_{h H}^{F B}$ & $\underline{q}$ & $\underline{q}$ & $\left(q_{l H}^{2}, \bar{q}\right)$ & $\left(q_{h H}^{2}, \bar{q}\right)$ \\
\hline 1.1.d & $x_{l L}=x_{l L}^{F B}>x_{l H}=x_{l H}^{\max }>x_{h L}^{\min }=x_{h L}>x_{h H}>x_{h H}^{F B}$ & $\underline{q}$ & $\underline{q}$ & $\left(q_{l H}^{2}, \bar{q}\right)$ & $\left(q_{h H}^{2}, \bar{q}\right)$ \\
\hline 1.1.e & $x_{l L}=x_{l L}^{F B}>x_{l H}>x_{h L}=x_{h H}>x_{h H}^{F B}$ & $\underline{q}$ & $\underline{q}$ & $\left(q_{l H}^{2}, q_{h H}\right)$ & $\left(q_{h H}^{2}, \bar{q}\right)$ \\
\hline \multicolumn{6}{|c|}{ Condition: $x_{h H}^{F B}\left[\Delta \theta_{1}-\Delta \theta_{2} q_{h H}^{2}\right]>x_{l H}^{F B}\left[\Delta \theta_{1}-\Delta \theta_{2} \bar{q}\right]$} \\
\hline $1.2 . \mathrm{a}^{*}$ & $x_{k}=x_{k}^{F B}$ & $\underline{q}$ & $\underline{q}$ & $\left(q_{l H}^{2}, \bar{q}\right)$ & $\left(q_{h H}^{2}, \bar{q}\right)$ \\
\hline $1.2 . b^{*}$ & $x_{l L}=x_{l L}^{F B}>x_{h L}^{F B}>x_{h L}>x_{l H}>x_{l H}^{F B}>x_{h H}=x_{h H}^{F B}$ & $\underline{q}$ & $\underline{q}$ & $\left(q_{l H}^{2}, \bar{q}\right)$ & $\left(q_{h H}^{2}, \bar{q}\right)$ \\
\hline $1.2 . \mathrm{c}^{*}$ & $x_{l L}=x_{l L}^{F B}>x_{h L}^{F B}>x_{h L}=x_{l H}>x_{l H}^{F B}>x_{h H}=x_{h H}^{F B}$ & $\bar{q}$ & $\underline{\bar{q}}$ & $\left(q_{l H}^{2}, \bar{q}\right)$ & $\left(q_{h H}^{2}, \bar{q}\right)$ \\
\hline
\end{tabular}

Other relevant solutions are 1.1.b, 1.1.c, 1.1.d and 1.1.e

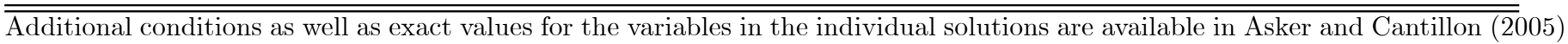
as well as in a separate appendix available on our webpages.

* Under the condition that $\Delta \theta_{1}-\Delta \theta_{2} \bar{q} \geq 0$ we can tighten the bound on $q_{h H}$ so that $q_{h H} \in\left(q_{h H}^{2}, q_{l H}\right)$

Table 2: Probabilities of winning and quality levels when $\Delta \theta_{1}-\Delta \theta_{2} \bar{q}<0$

\begin{tabular}{l|l|c|c|c|c|c}
\hline Solution & \multicolumn{1}{|c|}{ Probabilities of Winning $\left(x^{\prime} \mathrm{s}\right)$} & $q_{l L}$ & $q_{h L}$ & $q_{l H}$ & $q_{h H}$ \\
\hline Condition: & $x_{h H}^{F B}\left[\Delta \theta_{1}-\Delta \theta_{2} \bar{q}\right] \geq x_{l H}^{F B}\left[\Delta \theta_{1}-\Delta \theta_{2} q_{l H}^{2}\right]$ & \multicolumn{4}{|c}{} & \multicolumn{4}{|c}{} \\
\hline 2.1.a & \multicolumn{1}{c}{$x_{k}=x_{k}^{F B}$} & $\underline{q}$ & $q_{l H}^{2}$ & $\bar{q}$ \\
2.1.b & $x_{l L}=x_{l L}^{F B}>x_{h L}^{F B}>x_{h L}=x_{l H}>x_{l H}^{F B}>x_{h H}=x_{h H}^{F B}$ & $\underline{q}$ & $\underline{q}$ & $q_{l H}^{2}$ & $\bar{q}$ \\
2.1.c & $x_{l L}=x_{l L}^{F B}>x_{h L}=x_{h L}^{F B}>x_{l H}^{F B}>x_{l H}>x_{h H}>x_{h H}^{F B}$ & $\underline{q}$ & $\underline{q}$ & $\left(q_{l H}^{2}, q_{h H}\right)$ & $\left(q_{h H}^{2}, \bar{q}\right)$ \\
2.1.d & $x_{l L}=x_{l L}^{F B}>x_{h L}^{F B}>x_{l H}=x_{h L}>x_{l H}^{F B}>x_{h H}>x_{h H}^{F B}$ & $\underline{q}$ & $\underline{q}$ & $\left(q_{l H}^{2}, q_{h H}\right)$ & $\left(q_{h H}^{2}, \bar{q}\right)$ \\
2.1.e & $x_{l L}=x_{l L}^{F B}>x_{l H}>x_{h L}=x_{h H}>x_{h H}^{F B}$ & $\underline{q}$ & $\underline{q}$ & $\left(q_{l H}^{2}, q_{h H}\right)$ & $\left(q_{h H}^{2}, \bar{q}\right)$ \\
\hline
\end{tabular}

The relevant solutions are 1.2.a, 1.2.b, 1.2.c, 2.1.c, 2.1.d and 2.1.e

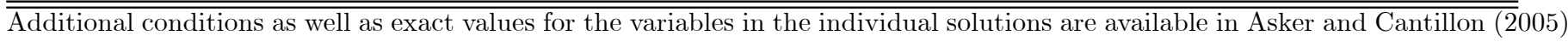
as well as in a separate appendix available on our webpages. 


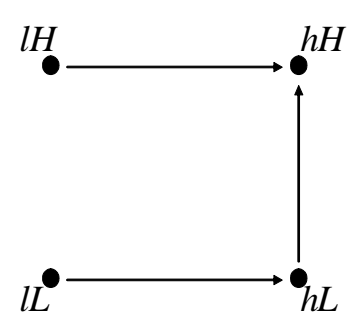

(a)

$\Delta \theta_{1}>\Delta \theta_{2} \bar{q}$

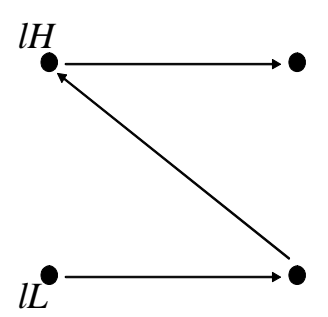

(b)

$\Delta \theta_{1}<\Delta \theta_{2} \bar{q}$

Figure 1: binding constraints in the efficient auction

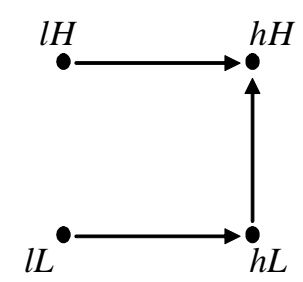

1.1.a

1.1.b

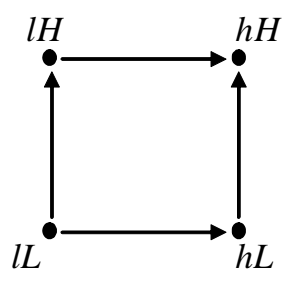

1.1.c

1.1.d

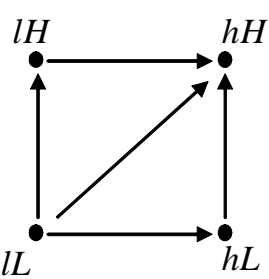

1.1.e

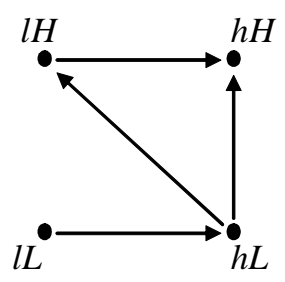

1.2.a

1.2.b

Figure 2: Binding IC constraints at the solution when $\Delta \theta_{1}-\Delta \theta_{2} \bar{q} \geq 0$

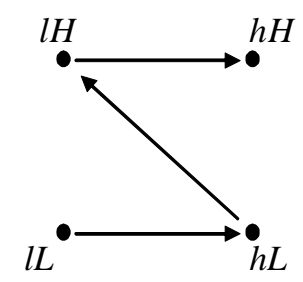

2.1.a

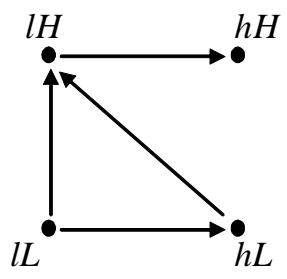

2.1.b

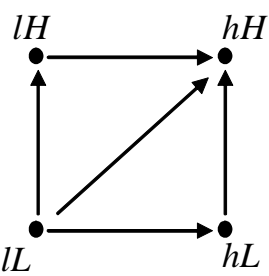

2.1.e

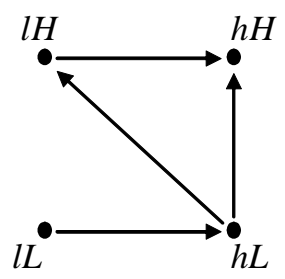

2.1.C

1.2.a

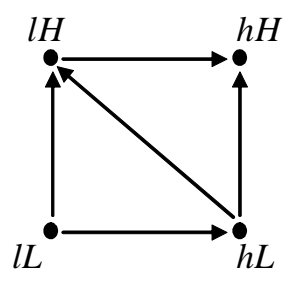

1.2.c

\section{2.b}

Figure 3: Binding IC constraints at the solution when $\Delta \theta_{1}-\Delta \theta_{2} \bar{q}<0$ 
Revenue by Mechanism

Probabilities: $\operatorname{Pr}(l H)=25, \operatorname{Pr}(h H)=25, \operatorname{Pr}(h L)=25, \operatorname{Pr}(l L)=25$

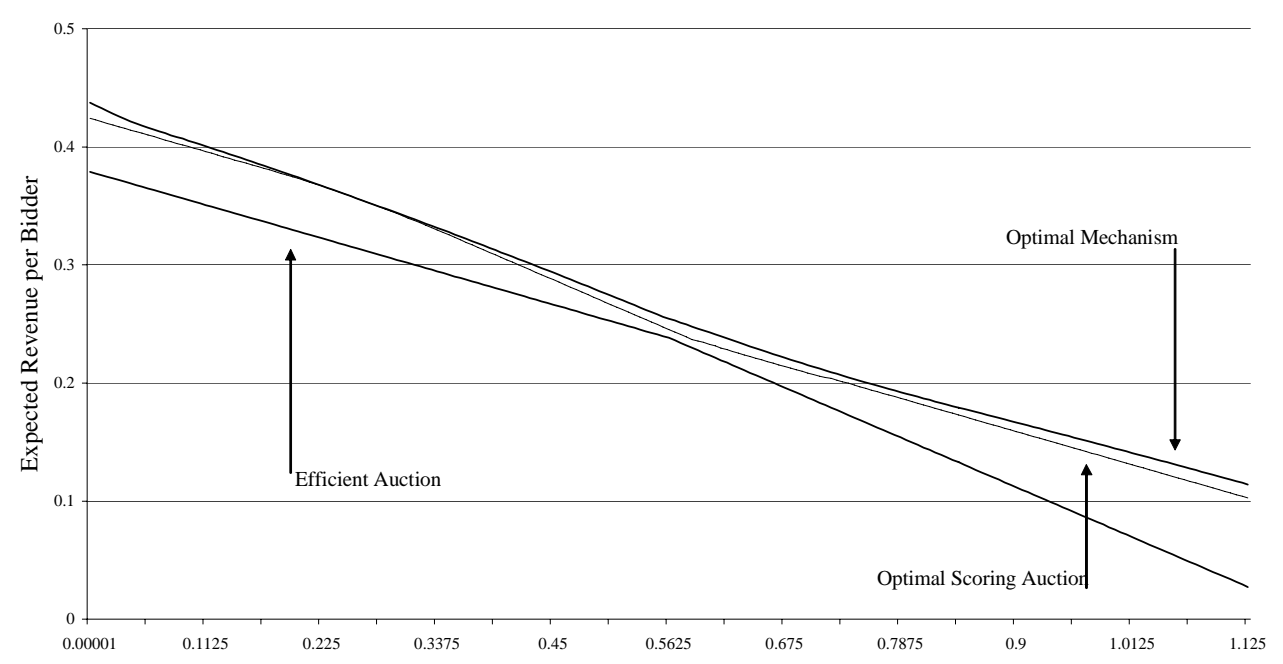

Figure 4
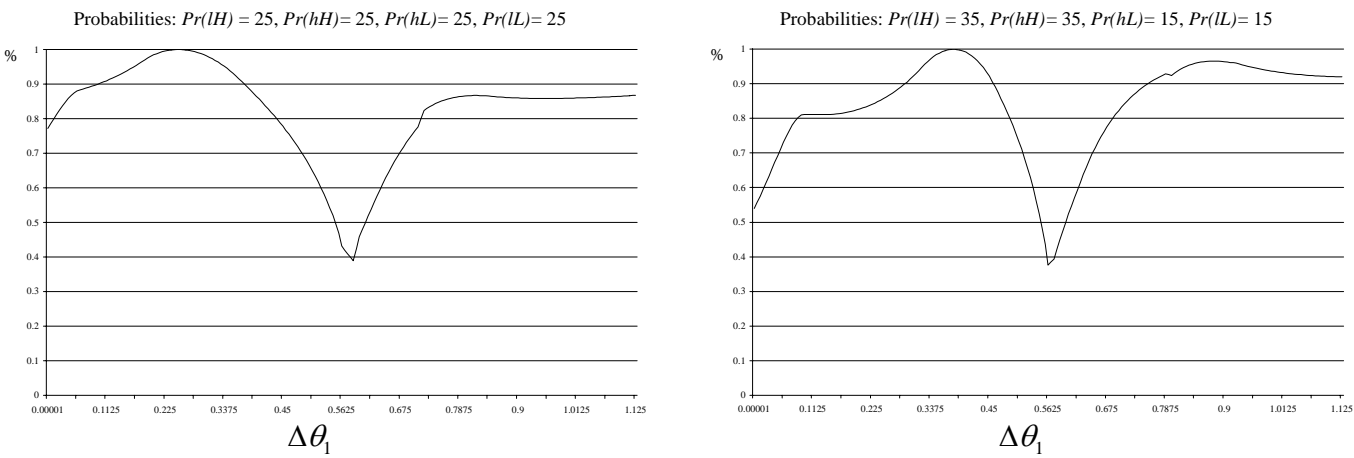

Probabilities: $\operatorname{Pr}(l H)=15, \operatorname{Pr}(h H)=35, \operatorname{Pr}(h L)=15, \operatorname{Pr}(L L)=35$

Probabilities: $\operatorname{Pr}(I H)=35, \operatorname{Pr}(h H)=15, \operatorname{Pr}(h L)=35, \operatorname{Pr}(I L)=15$
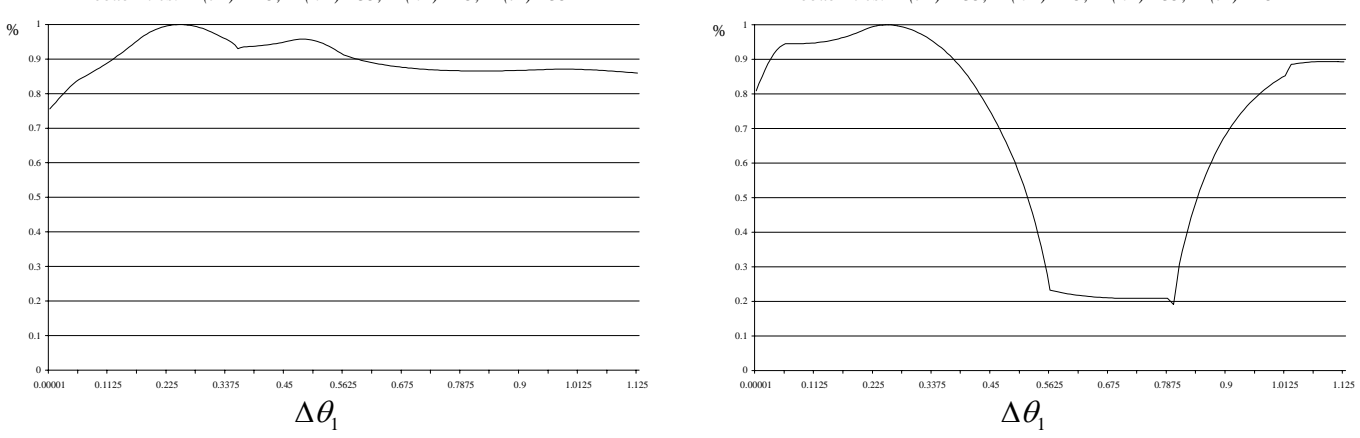

Figure 5: Proportion of (Optimal - Efficient Auction) 
Table 3: Proportion of the (opt. mech. - efficient auction) expected utility captured by the optimal scoring auction

\begin{tabular}{|c|c|c|c|c|c|c|c|c|c|c|c|}
\hline & \multirow{2}{*}{\multicolumn{4}{|c|}{ Probabilities }} & \multirow[t]{2}{*}{ Average } & \multirow[t]{2}{*}{$\operatorname{Max}$} & \multirow{2}{*}{$\begin{array}{l}\Delta \theta_{1} \text { at } \\
\text { maximum }\end{array}$} & \multirow[t]{2}{*}{ Min } & \multirow{2}{*}{$\begin{array}{l}\Delta \theta_{1} \text { at } \\
\text { minimum }\end{array}$} & \multicolumn{2}{|c|}{$\%$ of $\Delta \theta_{1}$ 's such that proportion is: } \\
\hline & & & & & & & & & & $>80 \%$ of opt. mech. & $<$ minimum +0.1 \\
\hline \multicolumn{12}{|c|}{ Core Parameter Values } \\
\hline 1 & 25 & 25 & 25 & 25 & 0.825 & 1.00 & 0.248 & 0.389 & 0.585 & 74.1 & 4.5 \\
\hline 2 & 20 & 30 & 20 & 30 & 0.874 & 1.00 & 0.248 & 0.739 & 0.574 & 86.6 & 19.4 \\
\hline 3 & 15 & 35 & 15 & 35 & 0.901 & 1.00 & 0.248 & 0.756 & 0.000 & 97.5 & 7.0 \\
\hline 4 & 30 & 20 & 30 & 20 & 0.761 & 1.00 & 0.248 & 0.284 & 0.686 & 62.2 & 13.4 \\
\hline 5 & 35 & 15 & 35 & 15 & 0.685 & 1.00 & 0.248 & 0.191 & 0.799 & 52.7 & 22.4 \\
\hline 6 & 20 & 20 & 30 & 30 & 0.812 & 1.00 & 0.180 & 0.423 & 0.596 & 68.2 & 5.0 \\
\hline 7 & 15 & 15 & 35 & 35 & 0.796 & 1.00 & 0.124 & 0.449 & 0.630 & 47.3 & 7.0 \\
\hline 8 & 30 & 30 & 20 & 20 & 0.835 & 1.00 & 0.315 & 0.372 & 0.574 & 74.1 & 3.5 \\
\hline 9 & 35 & 15 & 15 & 35 & 0.839 & 1.00 & 0.383 & 0.376 & 0.563 & 75.6 & 3.5 \\
\hline 10 & 20 & 30 & 30 & 20 & 0.779 & 1.00 & 0.248 & 0.465 & 0.709 & 44.8 & 14.9 \\
\hline 11 & 15 & 35 & 35 & 15 & 0.778 & 1.00 & 0.248 & 0.558 & 0.833 & 41.3 & 26.9 \\
\hline 12 & 30 & 20 & 20 & 30 & 0.892 & 1.00 & 0.248 & 0.670 & 0.518 & 83.6 & 13.4 \\
\hline 13 & 35 & 15 & 15 & 35 & 0.948 & 1.00 & 0.248 & 0.809 & 0.000 & 100 & 20.4 \\
\hline \multicolumn{12}{|c|}{ Extensions } \\
\hline 14 & 10 & 40 & 10 & 40 & 0.823 & 1.00 & 0.248 & 0.593 & 1.125 & 56.7 & 25.8 \\
\hline 15 & 40 & 10 & 40 & 10 & 0.604 & 1.00 & 0.248 & 0.127 & 0.810 & 45.7 & 31.8 \\
\hline 16 & 10 & 10 & 40 & 40 & 0.786 & 1.00 & 0.068 & 0.442 & 0.698 & 40.8 & 6.5 \\
\hline 17 & 40 & 40 & 10 & 10 & 0.836 & 1.00 & 0.450 & 0.358 & 0.563 & 60.2 & 4.5 \\
\hline 18 & 45 & 45 & 5 & 5 & 0.826 & 1.00 & 0.506 & 0.209 & 0.000 & 50.2 & 2.0 \\
\hline 19 & 15 & 25 & 30 & 30 & 0.817 & 1.00 & 0.153 & 0.535 & 0.038 & 51.2 & 7.5 \\
\hline 20 & 16 & 23 & 41 & 20 & 0.752 & 1.00 & 0.180 & 0.463 & 0.821 & 39.3 & 13.4 \\
\hline
\end{tabular}

\begin{tabular}{c|cccc|c|cc|cc|c|c}
21 & 25 & 25 & 25 & 25 & 0.825 & 1.00 & 0.028 & 0.389 & 0.065 & 74.1 & 4.5 \\
22 & 15 & 35 & 15 & 35 & 0.901 & 1.00 & 0.028 & 0.756 & 0.000 & 97.5 & 7.0 \\
23 & 35 & 15 & 35 & 15 & 0.685 & 1.00 & 0.028 & 0.191 & 0.088 & 52.7 & 22.4 \\
24 & 15 & 15 & 35 & 35 & 0.796 & 1.00 & 0.014 & 0.449 & 0.070 & 47.3 & 7.0 \\
25 & 35 & 35 & 15 & 15 & 0.839 & 1.00 & 0.043 & 0.376 & 0.063 & 75.6 & 3.5 \\
\hline
\end{tabular}

$\begin{array}{llllllllll} & \text { Robustness: } b=0.7\end{array}$

\begin{tabular}{c|cccc|c|cc|c|c|c|c}
26 & 25 & 25 & 25 & 25 & 0.737 & 1.00 & 0.285 & 0.429 & 2.078 & 49.3 & 24.9 \\
\hline \multicolumn{10}{c}{ Robustness: $\Delta \theta_{2}=2$} \\
27 & 25 & 25 & 25 & 25 & 0.751 & 1.00 & 0.180 & $0.409 \quad 0.765$ & 53.2 & 19.9 \\
\hline \multicolumn{10}{c}{ Robustness: $l=2$} \\
38 & 25 & 25 & 25 & 25 & 0.827 & 1.00 & 0.248 & 0.389 & 0.585 & 74.6 & 4.0 \\
\hline \hline
\end{tabular}

Notes: Each experiments sets the probabilites of each type (ordered $\alpha_{l H}, \alpha_{h H}, \alpha_{h L}, \alpha_{l L}$ ), then computes the expected utility for the optimal mechanism, the efficient auction and the optimal scoring auction for 201 values of $\Delta \theta_{1}$ covering the full range of the parameter values allowed by the model. 
Table 4: Percentage of the (optimal mechanism - efficient auction) expected utility captured by the simple scoring rule corresponding to an adjusted efficient auction

\begin{tabular}{|c|c|c|c|c|c|c|c|c|c|}
\hline & \multirow{2}{*}{\multicolumn{4}{|c|}{ Probabilities }} & \multirow[t]{2}{*}{ Average } & \multicolumn{2}{|c|}{ No Distortion to Allocation } & \multicolumn{2}{|c|}{ Distortion to Allocation } \\
\hline & & & & & & Max & Min & $\operatorname{Max}$ & Min \\
\hline & \multicolumn{4}{|c|}{$a^{*}=(0.95) a$} & & & & & \\
\hline 1 & 25 & 25 & 25 & & 0.223 & 38.3 & -19.5 & 78.1 & 72.9 \\
\hline 2 & 20 & 30 & 20 & 30 & 0.225 & 39.8 & -7.8 & 76.5 & 71.2 \\
\hline 3 & 15 & 35 & 15 & 35 & 0.218 & 36.6 & -1.6 & 71.9 & 68.5 \\
\hline 4 & 30 & 20 & 30 & 20 & 0.237 & 36.3 & -32.3 & 79.3 & 63.1 \\
\hline 5 & 35 & 15 & 35 & 15 & 0.242 & 34.0 & -49.3 & 80.5 & 38.0 \\
\hline 6 & 20 & 20 & 30 & 30 & 0.286 & 30.0 & -20.4 & 73.8 & 66.1 \\
\hline 7 & 15 & 15 & 35 & 35 & 0.339 & 20.5 & -25.5 & 68.7 & 58.7 \\
\hline 8 & 30 & 30 & 20 & 20 & 0.174 & 46.4 & -23.3 & 81.9 & 79.2 \\
\hline 9 & 35 & 15 & 15 & 35 & 0.114 & 53.8 & -37.9 & 85.0 & 84.4 \\
\hline 10 & 20 & 30 & 30 & 20 & 0.208 & 40.2 & -10.5 & 68.6 & 56.3 \\
\hline 11 & 15 & 35 & 35 & 15 & 0.186 & 41.9 & -2.6 & 55.5 & 33.8 \\
\hline 12 & 30 & 20 & 20 & 30 & 0.254 & 35.8 & -19.7 & 85.5 & 76.5 \\
\hline \multirow[t]{2}{*}{13} & 35 & 15 & 15 & 35 & 0.277 & 25.0 & -17.4 & 88.5 & 79.0 \\
\hline & \multicolumn{4}{|c|}{$a^{*}=(0.9) a$} & & & & & \\
\hline 1 & 25 & 25 & 25 & 25 & 0.195 & 36.2 & 1.4 & 82.3 & 80.6 \\
\hline 2 & 20 & 30 & 20 & 30 & 0.194 & 32.1 & 2.2 & 80.2 & 78.2 \\
\hline 3 & 15 & 35 & 15 & 35 & 0.189 & 28.3 & 3.3 & 75.1 & 74.6 \\
\hline 4 & 30 & 20 & 30 & 20 & 0.187 & 36.3 & 0.5 & 84.3 & 75.4 \\
\hline 5 & 35 & 15 & 35 & 15 & 0.170 & 36.8 & -3.4 & 81.8 & 69.2 \\
\hline 6 & 20 & 20 & 30 & 30 & 0.170 & 29.6 & 1.0 & 78.5 & 76.0 \\
\hline 7 & 15 & 15 & 35 & 35 & 0.145 & 23.8 & 0.3 & 74.8 & 71.5 \\
\hline 8 & 30 & 30 & 20 & 20 & 0.222 & 44.2 & 1.5 & 86.1 & 85.4 \\
\hline 9 & 35 & 35 & 15 & 15 & 0.253 & 54.6 & 1.4 & 90.3 & 89.8 \\
\hline 10 & 20 & 30 & 30 & 20 & 0.199 & 36.2 & 3.2 & 73.2 & 68.9 \\
\hline 11 & 15 & 35 & 35 & 15 & 0.200 & 36.3 & 6.3 & 60.7 & 52.9 \\
\hline 12 & 30 & 20 & 20 & 30 & 0.185 & 29.6 & 0.3 & 88.3 & 81.6 \\
\hline 13 & 35 & 15 & 15 & 35 & 0.170 & 21.4 & -1.2 & 87.0 & 82.4 \\
\hline
\end{tabular}


Probabilities: $\operatorname{Pr}(l H)=25, \operatorname{Pr}(h H)=25, \operatorname{Pr}(h L)=25, \operatorname{Pr}(I L)=25, \mathrm{a}^{*}=(0.95) \mathrm{a}$

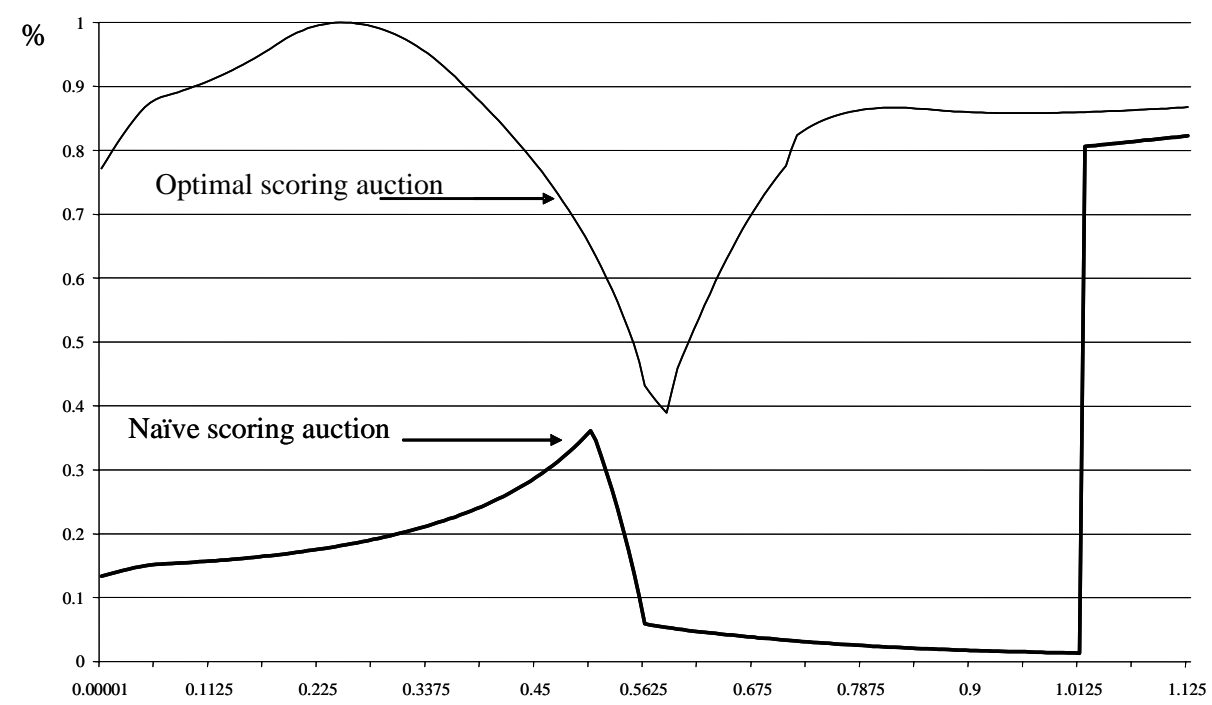

Figure 6: Performance of nave scoring auctions

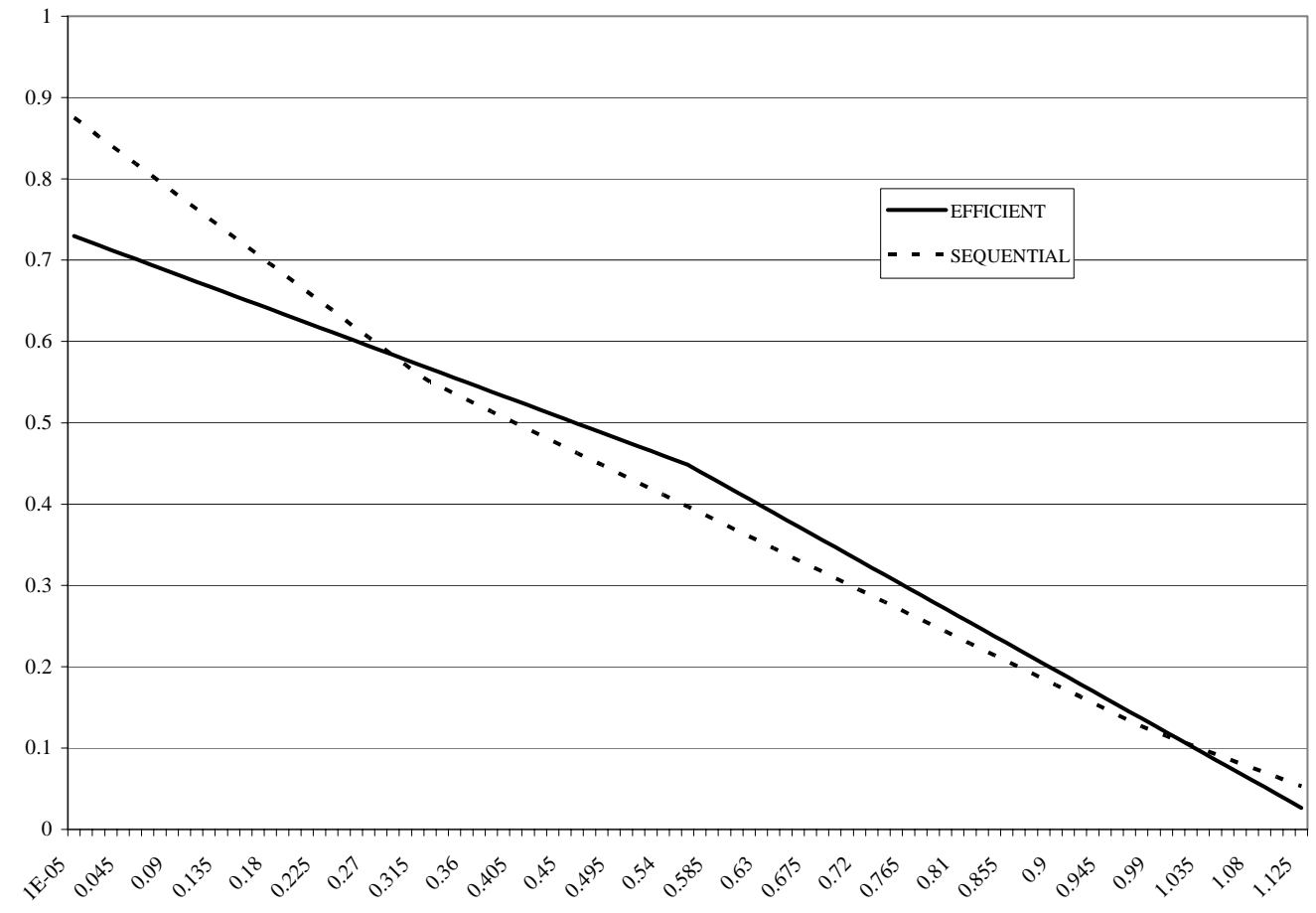

Figure 7: Performance of the optimal sequential procedure and the efficient auction for different values of $\Delta \theta_{1}$ when $v(q)=3 \sqrt{q}, \underline{\theta}_{1}=\underline{\theta}_{2}=1, \bar{\theta}_{2}=2, \alpha_{l H}=\alpha_{h L}=0.35$ and $\alpha_{l L}=\alpha_{h H}=0.15$ 
Table 6: Proportion of the (optimal mechanism - efficient auction) expected utility captured by the optimal sequential procedure*

\begin{tabular}{|c|c|c|c|c|c|c|c|c|c|c|c|}
\hline & \multicolumn{4}{|c|}{ Probabilities } & \multirow{2}{*}{$\begin{array}{l}\text { Average } \\
-152.7 \%\end{array}$} & \multirow{2}{*}{$\begin{array}{l}\text { Max } \\
100 \% \\
\end{array}$} & \multirow{2}{*}{$\begin{array}{l}\Delta \theta_{1} \\
\text { at max } \\
0\end{array}$} & \multirow{2}{*}{$\begin{array}{l}\text { Min } \\
-504.4 \%\end{array}$} & \multirow{2}{*}{$\begin{array}{l}\Delta \theta_{1} \\
\text { at min } \\
0.5625\end{array}$} & \multirow{2}{*}{$\begin{array}{l}\% \Delta \theta_{1} \\
\text { s.t. }>80 \% \\
6.9\end{array}$} & \multirow{2}{*}{$\begin{array}{l}\% \Delta \theta_{1} \\
\text { s.t. }>0 \% \\
20.8\end{array}$} \\
\hline 1 & 25 & 25 & 25 & 25 & & & & & & & \\
\hline 2 & 20 & 30 & 20 & 30 & $-78.2 \%$ & $100 \%$ & 0 & $-279.6 \%$ & 0.5625 & 7.9 & 23.8 \\
\hline 3 & 15 & 35 & 15 & 35 & $-7.2 \%$ & $100 \%$ & 0 & $-83.6 \%$ & 0.5625 & 7.9 & 35.6 \\
\hline 4 & 10 & 40 & 10 & 40 & $55.9 \%$ & $100 \%$ & 0 & $39.2 \%$ & 0.5625 & 8.9 & 100 \\
\hline 5 & 30 & 20 & 30 & 20 & $-238.7 \%$ & $100 \%$ & 0 & $-663.9 \%$ & 0.66375 & 5.9 & 18.8 \\
\hline 6 & 35 & 15 & 35 & 15 & $-365.0 \%$ & $100 \%$ & 0 & $-942.2 \%$ & 0.7875 & 5.0 & 15.8 \\
\hline 7 & 40 & 10 & 40 & 10 & $-591.8 \%$ & $100 \%$ & 0 & $-1,446.7 \%$ & 0.9 & 5.0 & 13.9 \\
\hline 8 & 20 & 20 & 30 & 30 & $-126.4 \%$ & $100 \%$ & 0 & $-404.0 \%$ & 0.5625 & 5.9 & 22.8 \\
\hline 9 & 15 & 15 & 35 & 35 & $-107.6 \%$ & $100 \%$ & 0 & $-356.2 \%$ & 0.5625 & 5.9 & 21.8 \\
\hline 10 & 10 & 10 & 40 & 40 & $-95.5 \%$ & $100 \%$ & 0 & $-284.4 \%$ & 0.5625 & 5.0 & 18.8 \\
\hline 11 & 30 & 30 & 20 & 20 & $-198.6 \%$ & $100 \%$ & 0 & $-746.5 \%$ & 0.5625 & 6.9 & 18.8 \\
\hline 12 & 35 & 35 & 15 & 15 & $-280.2 \%$ & $100 \%$ & 0 & $-1.302 .9 \%$ & 0.5625 & 6.9 & 14.9 \\
\hline 13 & 40 & 40 & 10 & 10 & $-394.1 \%$ & $100 \%$ & 0 & $-1,961.4 \%$ & 0.5625 & 6.0 & 9.9 \\
\hline 14 & 20 & 30 & 30 & 20 & $-147.2 \%$ & $100 \%$ & 0 & $-384.1 \%$ & 0.66375 & 7.9 & 25.7 \\
\hline 15 & 15 & 35 & 35 & 15 & $-108.2 \%$ & $100 \%$ & 0 & $-276.6 \%$ & 0.7875 & 10.9 & 32.7 \\
\hline 16 & 10 & 40 & 40 & 10 & $-46.1 \%$ & $100 \%$ & 0 & $-164.7 \%$ & 0.9 & 15.8 & 40.6 \\
\hline 17 & 30 & 20 & 20 & 30 & $-129.3 \%$ & $100 \%$ & 0 & $-411.4 \%$ & 0.5625 & 5.9 & 16.8 \\
\hline 18 & 35 & 15 & 15 & 35 & $-93.9 \%$ & $100 \%$ & 0 & $-244.8 \%$ & 0.5625 & 5.0 & 13.9 \\
\hline 19 & 40 & 10 & 10 & 40 & $-61.4 \%$ & $100 \%$ & 0 & $-142 . \%$ & 0.5625 & 4.0 & 13.9 \\
\hline
\end{tabular}

*Each experiment sets the value of the $\alpha_{k}$ 's (ordered $\alpha_{l H}, \alpha_{h H}, \alpha_{h L}, \alpha_{l L}$ ) and computes the expected utility from the optimal mechanism, the optimal sequential auction and the efficient auction for all the values for $\Delta \theta_{1}$ allowed by the model. Each experiment samples 101 equally spaced values for $\Delta \theta_{1}$ 


\section{Proofs: Not For Publication}




\section{Appendix Z: Proof of Theorem 1}

\section{Set-up of the Optimal Program}

For future reference, this appendix reproduces the optimization problem of the buyer with $U_{h H}=0$ (Lemma 2) and with the subset of the IC constraints that happen to bind at the optimum.

$\max _{\left\{x_{k}, q_{k}, U_{k}\right\}} \alpha_{l H}\left[x_{l H} W_{l H}\left(q_{l H}\right)-U_{l H}\right]+\alpha_{h H} x_{h H} W_{h H}\left(q_{h H}\right)+\alpha_{h L}\left[x_{h L} W_{h L}\left(q_{h L}\right)-U_{h L}\right]+\alpha_{l L}\left[x_{l L} W_{l L}\left(q_{l L}\right)-U_{l L}\right]$ subject to:

$$
\begin{array}{rlr}
U_{l H} & \geq x_{h H} \Delta \theta_{1} & \text { (IC } 1) \\
U_{h L} & \geq U_{l H}-x_{l H}\left[W_{l H}\left(q_{l H}\right)-W_{h L}\left(q_{l H}\right)\right] & \text { (IC 2) } \\
U_{h L} & \geq x_{h H} q_{h H} \Delta \theta_{2} & \text { (IC 3) } \\
U_{l L} & \geq U_{l H}+x_{l H} q_{l H} \Delta \theta_{2} & \text { (IC 4) } \\
U_{l L} & \geq U_{h L}+x_{h L} \Delta \theta_{1} & \text { (IC 5) } \\
U_{l L} & \geq U_{h H}+x_{h H} \Delta \theta_{1}+x_{h H} q_{h H} \Delta \theta_{2} & \text { (IC 6) } \\
N \sum_{k \in K} \alpha_{k} x_{k} & \leq 1-\left(1-\sum_{k \in K} \alpha_{k}\right)^{N} \text { for all subsets } K \text { of }\{l H, h H, h L, l L\} & \text { (feasibility) }
\end{array}
$$

(We omit the non exclusion constraint). The associated Lagrangian is given by:

$$
\begin{aligned}
& \alpha_{l H}\left[x_{l H} W_{l H}\left(q_{l H}\right)-U_{l H}\right]+\alpha_{h H} x_{h H} W_{h H}\left(q_{h H}\right)+\alpha_{h L}\left[x_{h L} W_{h L}\left(q_{h L}\right)-U_{h L}\right]+\alpha_{l L}\left[x_{l L} W_{l L}\left(q_{l L}\right)-U(\mathbb{I})\right. \\
& +\lambda_{1}\left[U_{l H}-x_{h H} \Delta \theta_{1}\right]+\lambda_{2}\left[U_{h L}-U_{l H}+x_{l H}\left(W_{l H}\left(q_{l H}\right)-W_{h L}\left(q_{l H}\right)\right)\right] \\
& +\lambda_{3}\left[U_{h L}-x_{h H} q_{h H} \Delta \theta_{2}\right]+\lambda_{4}\left[U_{l L}-U_{l H}-x_{l H} q_{l H} \Delta \theta_{2}\right]+\lambda_{5}\left[U_{l L}-U_{h L}-x_{h L} \Delta \theta_{1}\right] \\
& \lambda_{6}\left[U_{l L}-x_{h H} \Delta \theta_{1}-x_{h H} q_{h H} \Delta \theta_{2}\right]-\sum \gamma_{K}\left[N \sum_{k \in K} \alpha_{k} x_{k}-1+\left(1-\sum_{k \in K} \alpha_{k}\right)^{N}\right]
\end{aligned}
$$

(where $\lambda_{i}$ is the Lagrangian multiplier associated with IC constraint $i$, and $\gamma_{K}$ is the multiplier associated with feasibility constraint $K$ ). Figure 13 provides a graphical representation of these IC constraints together with their associated multipliers. A dotted line means that a constraint may bind at the optimum. A full line means it always binds.

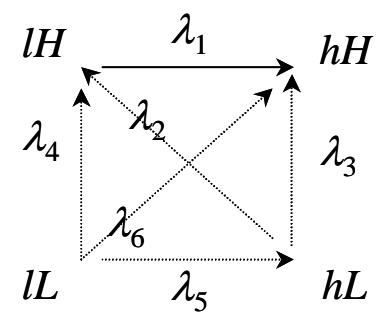

Figure 13: Potentially binding constraints at the solution 
The Kuhn-Tucker conditions of this program are standard. For future reference, we only reproduce those with respect to $U_{k}$ :

$$
\begin{aligned}
\lambda_{1}-\lambda_{2}-\lambda_{4} & =\alpha_{l H} \\
\lambda_{2}+\lambda_{3}-\lambda_{5} & =\alpha_{h L} \\
\lambda_{4}+\lambda_{5}+\lambda_{6} & =\alpha_{l L}
\end{aligned}
$$

\section{Characterization of the Optimal Buying Mechanism}

\section{Preliminaries}

We first define the notation that we will be using for some of the $x_{k}$ variables when they take specific values. When $x_{l H}$ takes its maximum value conditional on $l L$ keeping priority in the contract allocation, we will denote it $x_{l H}^{\max }$. Formally, $x_{l H}^{\max }$ is defined by the equation

$$
N\left(\alpha_{l H} x_{l H}^{\max }+\alpha_{l L} x_{l L}^{F B}\right)=1-\left(\alpha_{h L}+\alpha_{h H}\right)^{N}
$$

By Border (1991), this implies the following allocation: When there is a type $l L$, give the contract to $l L$, if not, give priority to a type $l H$ if there is one. Conversely, $x_{h L}^{\min }$ corresponds to the expected probability of winning for $h L$ when $l H$ and $l L$ have priority over $h L$ (but $h L$ maintains priority over $h H)$. Formally,

$$
N\left(\alpha_{l H} x_{l H}^{\max }+\alpha_{h L} x_{h L}^{\min }+\alpha_{l L} x_{l L}^{F B}\right)=1-\alpha_{h H}^{N}
$$

Finally, $\bar{x}$ is defined such that $x_{l H}=x_{h L}$ and they have priority over $h H$ in the allocation, that is

$$
N\left(\left(\alpha_{l H}+\alpha_{h L}\right) \bar{x}+\alpha_{l L} x_{l L}^{F B}\right)=1-\alpha_{h H}^{N}
$$

The proof of Theorem 1 uses the following result repeatedly:

Lemma 7: Suppose $U_{l H}=x_{h H} \Delta \theta_{1}$. (1) Suppose further that $U_{h L, l H} \geq U_{h L, h H}$. Then, $x_{h L}>x_{l H}$ if and only if $U_{l L, h L}>U_{l L, l H}$. (2) Suppose now that $U_{h L, l H} \leq U_{h L, h H}$. Then $U_{l L, h L} \geq U_{l L, l H}$ when $x_{h L} \geq x_{l H}$.

Proof: The result follows directly from a comparison of $U_{l L, l H}$ and $U_{l L, h L}$ (when $\left.U_{h L, l H} \geq U_{h L, h H}\right)$ :

$$
U_{l L, l H}=x_{l H} q_{l H} \Delta \theta_{2}+x_{h H} \Delta \theta_{1} \quad U_{l L, h L}=x_{h L} \Delta \theta_{1}-x_{l H} \Delta \theta_{1}+x_{l H} q_{l H} \Delta \theta_{2}+x_{h H} \Delta \theta_{1}
$$

When $U_{h L, h H} \geq U_{h L, l H}, U_{l L, h L}=x_{h L} \Delta \theta_{1}+x_{h H} q_{h H} \Delta \theta_{2}$. Since $U_{h L, h H} \geq U_{h L, l H}$ is equivalent to $x_{h H}\left[W_{l H}\left(q_{h H}\right)-W_{h L}\left(q_{h H}\right)\right] \leq x_{l H}\left[W_{l H}\left(q_{l H}\right)-W_{h L}\left(q_{l H}\right)\right]$, the condition implies $U_{l L, h L} \geq U_{l L, l H}$ when $x_{h L}>x_{l H}$. QED. 
Lemma 8: Suppose that $I C_{h L, h H}$ is satisfied. Then $x_{h L} \geq x_{h H} \Longrightarrow I C_{l L, h H}$ is satisfied.

Proof: $\mathrm{IC}_{h L, h H}$ satisfied means that $U_{l L, h L} \stackrel{\text { defn }}{=} U_{h L}+x_{h L} \Delta \theta_{1} \geq U_{h H}+x_{h H} \Delta \theta_{2} q_{h H}+x_{h L} \Delta \theta_{1}$. On this other hand, $U_{l L, h H}=U_{h H}+x_{h H} \Delta \theta_{2} q_{h H}+x_{h H} \Delta \theta_{1}$. Clearly, $U_{l L, h H} \leq U_{l L, h L}$ as long as $x_{h L} \geq x_{h H}$. QED

We are now ready to prove theorem 1 . The proof proceeds by progressively partioning the space of parameters into sets of parameters for which the solution shares the same binding IC and feasibility constraints. The logic of the proof is pretty simple, even if the mechanics can be involved. For this reason an exhaustive exposition of the proof of part I, scenario 1 is presented. The arguments in the rest of the proof are presented more briefly where they mirror those in part I, scenario 1.

Proof of part I of Theorem 1: $W_{l H}(\bar{q})-W_{h L}(\bar{q})>0$ i.e. $\Delta \theta_{1}>\bar{q} \Delta \theta_{2}$

The binding constraints in the buyer-optimal efficient mechanism are $\mathrm{IC}_{l H, h H}, \mathrm{IC}_{h L, h H}$ and $\mathrm{IC}_{l L, h L}$. The buyer's resulting expected utility is given by

$$
\begin{aligned}
& \alpha_{l H} x_{l H} W_{l H}\left(q_{l H}\right)+\alpha_{h H} x_{h H}\left[W_{h H}\left(q_{h H}\right)-\frac{\alpha_{l H}}{\alpha_{h H}} \Delta \theta_{1}-\frac{\alpha_{h L}+\alpha_{l L}}{\alpha_{h H}} q_{h H} \Delta \theta_{2}\right] \\
& +\alpha_{h L} x_{h L}\left[W_{h L}\left(q_{h L}\right)-\frac{\alpha_{l L}}{\alpha_{h L}} \Delta \theta_{1}\right]+\alpha_{l L} x_{l L} W_{l L}\left(q_{l L}\right)
\end{aligned}
$$

(where, again, we have highlighted the virtual welfares associated with each type). Keeping the probabilities fixed at $x_{k}=x_{k}^{F B}$, optimizing the $q$ 's in (5) requires that only $q_{h H}$ be adjusted away from the efficient level and set equal to

$$
q_{h H}^{2}=\arg \max \left\{W_{h H}\left(q_{h H}\right)-\frac{\alpha_{l H}}{\alpha_{h H}} \Delta \theta_{1}-\frac{\alpha_{h L}+\alpha_{l L}}{\alpha_{h H}} q_{h H} \Delta \theta_{2}\right\}
$$

This reduces the informational rents of $h L$ and $l L$. From Lemma $7(2)$, we know that $U_{l L, h L} \geq U_{l L, l H}$ as long as $U_{h L, h H} \geq U_{h L, l H}$. Hence, we need to consider only two scenarios:

Scenario 1: At $q_{h H}^{2}, U_{h L, h H} \geq U_{h L, l H}$, that is,

$$
x_{h H}^{F B}\left[W_{l H}\left(q_{h H}^{2}\right)-W_{h L}\left(q_{h H}^{2}\right)\right] \leq x_{l H}^{F B}\left[W_{l H}(\bar{q})-W_{h L}(\bar{q})\right]
$$

In this case, all IC constraints remain satisfied as we decrease $q_{h H}$ to $q_{h H}^{2}$.

We now consider the optimization of the probabilities of winning. From (5) and the model assumptions, the virtual welfare associated with $l L$ is the largest. Moreover, the virtual welfare associated with $l H$ is larger than that associated with $h H$. Thus, we need to consider three cases depending on the relative ranking of the virtual welfare of $h L$ with respect to the virtual welfares of $h H$ and $l H$. 
1. $V W_{h L} \geq V W_{l H} \geq V W_{h H}: W_{h L}(\underline{q})-\frac{\alpha_{l L}}{\alpha_{h L}} \Delta \theta_{1} \geq W_{l H}(\bar{q})>W_{h H}\left(q_{h H}^{2}\right)-\frac{\alpha_{l H}}{\alpha_{h H}} \Delta \theta_{1}-\frac{\alpha_{h L}+\alpha_{l L}}{\alpha_{h H}} q_{h H}^{2} \Delta \theta_{2}$ [Solution 1.1.a]

The optimal probabilities of winning are $x_{k}=x_{k}^{F B}$ since the ranking of the virtual welfares corresponds to the ranking of the first best welfares. All IC constraints are satisfied given the arguments above. The $x$ 's and $q$ 's are optimized given the binding constraints; $q_{l H}=\bar{q}$, $q_{h H}=q_{h H}^{2}$ and $q_{h L}=q_{l L}=\underline{q}$.

2. $V W_{l H}>V W_{h L} \geq V W_{h H}: W_{l H}(\bar{q})>W_{h L}(\underline{q})-\frac{\alpha_{l L}}{\alpha_{h L}} \Delta \theta_{1} \geq W_{h H}\left(q_{h H}^{2}\right)-\frac{\alpha_{l H}}{\alpha_{h H}} \Delta \theta_{1}-\frac{\alpha_{h L}+\alpha_{l L}}{\alpha_{h H}} q_{h H}^{2} \Delta \theta_{2}$ In this case, type $l H$ generates a higher level of virtual welfare than type $h L$. Thus, the buyer would rather give the contract to supplier $l H$ than to supplier $h L$, i.e. he would like to change the order of priority in the allocation. Increasing $x_{l H}$ while decreasing $x_{h L}$ concurrently (keeping $\alpha_{l H} x_{l H}+\alpha_{h L} x_{h L}+\alpha_{l L} x_{l L}^{F B}$ constant) does not initially affect any of the virtual welfare and it increases the buyer's expected utility. This process continues until either a new IC constraint binds or we have reach $x_{l H}=x_{l H}^{\max }$.

We now argue that the only potentially new binding constraint is $\mathrm{IC}_{l L, l H}$. To see this consider the following:

(a) $\underline{h L}$ 's IC constraints: Given that $U_{h L, l H}=U_{l H}-x_{l H}\left[\Delta \theta_{1}-\Delta \theta_{2} \bar{q}\right]$ and that $U_{l H}$ is not affected by the process, the incentives for $h L$ to imitate $l H$ have actually decreased. $\mathrm{IC}_{h L, l L}$ remain satisfied as well since $\mathrm{IC}_{l L, h L}$ is binding and $x_{l L}>x_{h L}$.

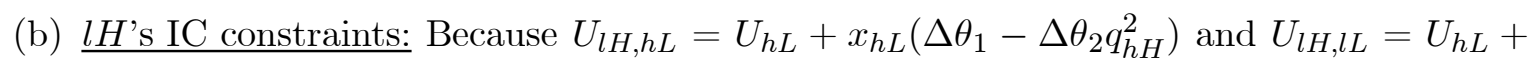
$x_{h L} \Delta \theta_{1}-x_{l L} \Delta \theta_{2} \underline{q}$, the incentives for $l H$ to imitate $h L$ and $l L$ have decreased $\left(U_{h L}=\right.$ $x_{h H} \Delta \theta_{1} q_{h H}^{2}$ is not affected by the process).

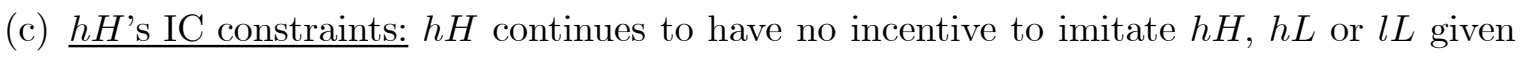
that $\mathrm{IC}_{l H, h H}$ and $\mathrm{IC}_{h L, h H}$ are binding, and $U_{h H, l L}$ is not affected by the process.

(d) $\underline{l L}$ 's IC constraint: By Lemma 8, $\mathrm{IC}_{l L, h H}$ is not affected by the process. By Lemma 7(2), $\mathrm{IC}_{l L, l H}$ remains satisfied as long as $x_{l H} \leq x_{h L}$, but it could start binding afterwards.

Thus, we continue to increase $x_{l H}$ at the cost of $x_{h L}$ until either $x_{l H}=x_{l H}^{\max }$ or $\mathrm{IC}_{l L, l H}$ starts binding, whichever comes first.

(a) $x_{l H}=x_{l H}^{\max }$ first. [Solution 1.1.b]

This means that $U_{l L, h L} \geq U_{l L, l H}$ even when $x_{l H}$ reaches its maximum. This corresponds to the solution because there are no more opportunities to increase the buyer's expected utility: the $q$ 's are optimized given the binding IC constraints, the $x$ 's are optimized 
given the virtual welfare and the feasibility constraints. The solution is thus: $q_{l H}=\bar{q}$, $q_{h H}=q_{h H}^{2}, q_{h L}=q_{l L}=\underline{q}$ and $x_{l L}=x_{l L}^{F B}>x_{l H}=x_{l H}^{\max }>x_{h L}=x_{h L}^{\min }>x_{h H}=x_{h H}^{F B}$. By the argument just above, all IC constraints are satisfied.

(b) $\mathrm{IC}_{l L, l H}$ starts binding. [Solution 1.1.c]

At that point, $U_{l L, l H}=U_{l L, h L}$, that is, $x_{h H}^{F B}\left[W_{l H}\left(q_{h H}^{2}\right)-W_{h L}\left(q_{h H}^{2}\right)\right]=x_{h L} \Delta \theta_{1}-x_{l H} \bar{q} \Delta \theta_{2}$ (note that by Lemma $7(2)$, this happens at $x_{l H}>x_{h L}$ ).

We now argue that we should be looking for a solution where both $\mathrm{IC}_{l L, h L}$ and $\mathrm{IC}_{l L, l H}$ are binding. Indeed, if only $\mathrm{IC}_{l L, l H}$ binds, the virtual welfare associated with $h L$ is $W_{h L}^{F B}$ which is greater than the virtual welfare associated with $l H$. Thus the buyer would want to set $x_{h L}$ back to $x_{h L}^{F B}$, but this would bring us back to the starting point.

Thus the buyer further increases his expected utility by increasing $x_{l H}$ and decreasing $x_{h L}$ while keeping $\alpha_{l H} x_{l H}+\alpha_{h L} x_{h L}+\alpha_{l L} x_{l L}^{F B}$ constant and $x_{h H}^{F B}\left[W_{l H}\left(q_{h H}\right)-W_{h L}\left(q_{h H}\right)\right]=$ $x_{h L} \Delta \theta_{1}-x_{l H} \bar{q} \Delta \theta_{2}$. This requires that we adjust $q_{h H}$ (specifically we need to increase $\left.q_{h H}\right)$.

A change in $q_{h H}$ corresponds to a change in the value of the Lagrangian multiplier on the $\mathrm{IC}_{l L, l H}$ constraint. Using the expressions in (1) to (4), we can rewrite the expressions for $l H$ and $h H$ 's virtual welfares as follows:

$$
\begin{aligned}
V W_{l H} & =\max _{q_{l H}}\left\{W_{l H}\left(q_{l H}\right)-\frac{\lambda_{4}}{\alpha_{l H}} q_{l H} \Delta \theta_{2}\right\} \\
V W_{h H} & =\max _{q_{h H}}\left\{W_{h H}\left(q_{h H}\right)-\frac{\alpha_{l H}+\lambda_{4}}{\alpha_{h H}} \Delta \theta_{1}-\frac{\alpha_{h L}+\alpha_{l L}-\lambda_{4}}{\alpha_{h H}} \Delta \theta_{2} q_{h H}\right\}
\end{aligned}
$$

where $\lambda_{4}$ is the Lagrangian multiplier on the $\mathrm{IC}_{l L, l H}$ constraint.

Thus, practically, we increase $x_{l H}$ and decrease $x_{h L}$ concurrently to keep $\alpha_{l H} x_{l H}+$ $\alpha_{h L} x_{h L}+\alpha_{l L} x_{l L}^{F B}$ constant. This implies a new value for $q_{h H}$ and $q_{l H}$ to ensure that $x_{h H}^{F B}\left[W_{l H}\left(q_{h H}\right)-W_{h L}\left(q_{h H}\right)\right]=x_{h L} \Delta \theta_{1}-x_{l H} q_{l H} \Delta \theta_{2}$. These correspond to a new value for $\lambda_{4}$ through (9). Specifically, $\lambda_{4}$ increases.

This process increases the virtual welfare associated with $h L, W_{h L}(\bar{q})-\frac{\alpha_{l L}-\lambda_{4}}{\alpha_{h L}} \Delta \theta_{1}$, and decreases the virtual welfare associated with $l H$ and $h H$ (see (8) and (9)).

It continues until we have either reached the upper bound to $x_{l H}, x_{l H}^{\max }$, or the virtual welfares associated with $l H$ and $h L$ become equal:

$$
\max _{q_{l H}}\left\{W_{l H}\left(q_{l H}\right)-\frac{\lambda_{4}^{*}}{\alpha_{l H}} q_{l H} \Delta \theta_{2}\right\}=W_{h L}(\underline{q})-\frac{\alpha_{l L}-\lambda_{4}^{*}}{\alpha_{h L}} \Delta \theta_{1}
$$

whichever comes first. Thus $\lambda_{4} \in\left(0, \lambda_{4}^{*}\right) \subset\left(0, \alpha_{l L}\right)$ as required by (4).

This defines the solution: $x_{l L}=x_{l L}^{F B}>x_{l H}^{\max } \geq x_{l H}>x_{h L} \geq x_{h L}^{\min }>x_{h H}=x_{h H}^{F B}$, $q_{l L}=q_{h L}=\underline{q}$ and $q_{l H}$ and $q_{h H}$ defined by (8) and (9), $q_{l H}, q_{h H}<\bar{q}$. The $x$ 's are 
optimized given the virtual welfares and the feasibility constraints. The $q$ 's are optimized given the binding constraints.

All IC constraints remain satisfied. The arguments for this are the same as those we made above, except for $\mathrm{IC}_{h L, l H}$, which follows because $x_{h H}^{F B}\left[W_{l H}\left(q_{h H}\right)-W_{h L}\left(q_{h H}\right)\right] \stackrel{U_{l L, h L}=U_{l L, l H}}{=}$ $x_{h L} \Delta \theta_{1}-x_{l H} q_{l H} \Delta \theta_{2}<x_{l H}\left[W_{l H}\left(q_{l H}\right)-W_{h L}\left(q_{l H}\right)\right]$ when $x_{l H}>x_{h L}$.

3. $V W_{l H} \geq V W_{h H}>V W_{h L}: W_{l H}(\bar{q})>W_{h H}\left(q_{h H}^{2}\right)-\frac{\alpha_{l H}}{\alpha_{h H}} \Delta \theta_{1}-\frac{\alpha_{h L}+\alpha_{l L}}{\alpha_{h H}} q_{h H}^{2} \Delta \theta_{2}>W_{h L}(\underline{q})-$ $\frac{\alpha_{l L}}{\alpha_{h L}} \Delta \theta_{1}$.

In this case, the ideal ordering of types in the allocation is $l L \succ l H \succ h H \succ h L$. The buyer increases his expected utility by decreasing $x_{h L}$, first to the benefit of $x_{l H}$ (that is, keeping $\alpha_{l H} x_{l H}+\alpha_{h L} x_{h L}+\alpha_{l L} x_{l L}^{F B}$ constant), and then to the benefit of $x_{h H}$ (that is, keeping $\left.N\left(\alpha_{l H} x_{l H}^{\max }+\alpha_{h L} x_{h L}+\alpha_{l L} x_{l L}^{F B}+\alpha_{h H} x_{h H}\right)=1\right)$.

This process initially does not affect any of the virtual welfares until a new IC constraint binds. By the same arguments as in point 2 above, we can establish that the first binding constraint is $\mathrm{IC}_{l L, l H}$. When it binds $x_{h H}\left[\Delta \theta_{1}-\Delta \theta_{2} q_{h H}^{2}\right]=x_{h L} \Delta \theta_{1}-x_{l H} \Delta \theta_{2} \bar{q}$. At this point, $x_{l H}>x_{h L}>x_{h H}$ (the first inequality comes from Lemma $7(2)$ ).

Once this happens, any further improvement requires that we keep $U_{l L, h L}=U_{l L, l H}$ (otherwise, if $U_{l L, h L}<U_{l L, l H}, \mathrm{IC}_{l L, h L}$ ceases to bind, the virtual welfare associated with $h L$ bounces back to $W_{h L}^{F B}$ and thus we get back to the starting point). We are thus in a similar situation as in point 2 above. Any further change in the $x$ 's requires some changes in the $q$ 's and thus in the value of the multiplier on the IC constraints. Using the expressions in (1) to (4), the resulting virtual welfares associated with $l H, h H$ and $h L$ are:

$$
\begin{aligned}
V W_{l H} & =\max _{q_{l H}}\left\{W_{l H}\left(q_{l H}\right)-\frac{\lambda_{4}}{\alpha_{l H}} \Delta \theta_{2} q_{l H}\right\} \\
V W_{h H} & =\max _{h H}\left\{W_{h H}\left(q_{h H}\right)-\frac{\left(\alpha_{l H}+\lambda_{4}\right)}{\alpha_{h H}} \Delta \theta_{1}-\frac{\left(\alpha_{h L}+\alpha_{l L}-\lambda_{4}\right)}{\alpha_{h H}} \Delta \theta_{2} q_{h H}\right\} \\
V W_{h L} & =W_{h L}(\underline{q})-\frac{\alpha_{l L}-\lambda_{4}}{\alpha_{h L}} \Delta \theta_{1}
\end{aligned}
$$

where $\lambda_{4} \in\left(0, \alpha_{l L}\right)$ is such that $U_{l L, h L}=U_{l L, l H}$ i.e. $x_{h H}\left[W_{l H}\left(q_{h H}\right)-W_{h L}\left(q_{h H}\right)\right]=x_{h L} \Delta \theta_{1}-$ $x_{l H} q_{l H} \Delta \theta_{2}$ for the current value of $x_{h L}$ ( $x_{l H}$ and $x_{h H}$ are well-defined once $x_{h L}$ is defined given that $l H$ has priority $h H$ is also clear). Practically, a decrease in $x_{h L}$ is associated with an increase in $q_{h H}$, a decrease in $q_{l H}$ and an increase in $\lambda_{4}$. This decreases $V W_{l H}$ and $V W_{h H}$ and increases $V W_{h L}$.

The difference relative to Solution 1.1.c is what ends this process. Here, the process ends 
when either a new IC constraint binds or the relative ranking of virtual welfare changes. ${ }^{1}$ The only new IC constraint that can bind is $\mathrm{IC}_{l L, h H}$. This happens at $x_{h L}=x_{h H}$. Thus we need to distinguish the following cases depending on which event happens first:

(a) We have reached $V W_{l H} \geq V W_{h H}=V W_{h L}$ and $x_{l H}=x_{l H}^{\max }$. Then this is the solution. The buyer is indifferent between $h H$ and $h L$. The qualities are given by the value of $\lambda_{4}$ that solves for $V W_{h H}=V W_{h L}$ in (11) and (12), $q_{l L}=q_{h L}=\underline{q}$ and $x_{l L}=x_{l L}^{F B}$, $x_{l H}=x_{l H}^{\max }>x_{h L}^{\min } \geq x_{h L}>x_{h H} \geq x_{h H}^{F B}$. [Solution 1.1.d]

(b) We have reached $V W_{l H} \geq V W_{h H}=V W_{h L}$ at $x_{l H}<x_{l H}^{\max }$. Then the buyer can further increase his expected utility by decreasing $x_{h L}$ and increasing $x_{l H}$ keeping $U_{l L, l H}=$ $U_{l L, h L}$. This further decreases $V W_{l H}$ and $V W_{h H}$ and increases $V W_{h L}$. The process stops when either $V W_{l H}=V W_{h L}$ or $x_{l H}=x_{l H}^{\max }$, whichever comes earlier. At the solution the $q$ 's are defined from (11) and (12) for the value of $\lambda_{4}$ at which the process stops, $q_{l L}=q_{h L}=\underline{q}$ and $x_{l L}=x_{l L}^{F B}, x_{l H}^{\max } \geq x_{l H}>x_{h L} \geq x_{h L}^{\min }$ and $x_{h H}=x_{h H}^{F B}$. This corresponds to Solution 1.1.c. above.

(c) We have reached $V W_{l H}=V W_{h H}>V W_{h L}$. (note that this implies that $q_{l H}<q_{h L}$ given (10) and (11)). The buyer further increases his expected utility by decreasing $x_{h L}$ and adjusting $x_{l H}$ and $x_{h H}$ in a way that preserves $V W_{l H}=V W_{h H}$ and $U_{l L, l H}=U_{l L, h L} \cdot{ }^{2}$ Thus $\lambda_{4}$ is fixed and the virtual welfares are not affected. This process continues until $x_{h L}=x_{h H}\left(<x_{l H}\right)$ at which point $U_{l L, h H}$ starts binding. At this stage we have:

$$
\begin{aligned}
U_{l L, l H} & =x_{h H} \Delta \theta_{1}+x_{l H} q_{l H} \Delta \theta_{2}=U_{l L, h H}=x_{h H} \Delta \theta_{1}+x_{h H} q_{h H} \Delta \theta_{2} \\
& =U_{l L, h L}=x_{h L} \Delta \theta_{1}+x_{h H} q_{h H} \Delta \theta_{2}
\end{aligned}
$$

Using the expressions in (1) to (4), the virtual welfares are given by

$$
\begin{aligned}
V W_{l H} & =\max _{q_{l H}}\left\{W_{l H}\left(q_{l H}\right)-\frac{\lambda_{4}}{\alpha_{l H}} \Delta \theta_{2} q_{l H}\right\} \\
V W_{h H} & =\max _{q_{h H}}\left\{W_{h H}-\frac{\alpha_{l H}+\lambda_{4}}{\alpha_{h H}} \Delta \theta_{1}-\frac{\alpha_{h L}+\alpha_{l L}-\lambda_{4}}{\alpha_{h H}} \Delta \theta_{2} q_{h H}\right\} \\
V W_{h L} & =W_{h L}(\underline{q})-\frac{\alpha_{l L}-\lambda_{4}-\lambda_{6}}{\alpha_{h L}} \Delta \theta_{1}
\end{aligned}
$$

where $\lambda_{4}$ and $\lambda_{6}$ are the multipliers on the $\mathrm{IC}_{l L, l H}$ and $\mathrm{IC}_{l L, h H}$ constraint respectively.

\footnotetext{
${ }^{1}$ No feasibility constraint binds in the process. Indeed, the only potential feasibility constraint would involve $x_{h H}$ hitting its maximum but this never occurs before $x_{h H}=x_{h L}$.

${ }^{2}$ The feasibility constraints on the $x$ 's are $N\left(\alpha_{l L} x_{l L}^{F B}+\alpha_{l H} x_{l H}+\alpha_{h H} x_{h H}\right) \leq 1-\alpha_{h L}^{N}$ and $N\left(\alpha_{l L} x_{l L}^{F B}+\alpha_{l H} x_{l H}+\right.$ $\left.\alpha_{h L} x_{h L}+\alpha_{h H} x_{h H}\right)=1$
} 
There exists a value for $\lambda_{4}$ and $\lambda_{6}$ such that $V W_{l H}=V W_{h H}=V W_{h L}$ and $U_{l L, l H}=$ $U_{l L, h L}=U_{l L, h H}$ and $N\left(\alpha_{l H} x_{l H}+\alpha_{h L} x_{h L}+\alpha_{l L} x_{l L}^{F B}+\alpha_{h H} x_{h H}\right)=1$. Indeed, we have five equations and five unknowns: $\lambda_{4}, \lambda_{6}, x_{l H}, x_{h L}$ and $x_{h H}$ (from (15) and the fact $V W_{l H}=V W_{h L}$, we know that $\alpha_{l L}-\lambda_{4}-\lambda_{6}>0$, thus $\alpha_{h L}+\alpha_{l L}-\lambda_{4}$ in (14) is ensured to be positive which is required by the non negative constraint on the multipliers).

These values for $\lambda_{4}$ and $\lambda_{6}$ correspond to the solution. At the solution, $x_{l H}>x_{h H}=x_{h L}$ (implied by $\left.U_{l L, l H}=U_{l L, h L}=U_{l L, h H}\right), q_{l H}<q_{h L}<\bar{q}$ and $q_{h L}=q_{h H}=\underline{q}$. The buyer is indifferent among $l H, h H$ and $h L$ and the $x$ 's are thus optimized. The $q$ 's are optimized given the binding constraints and the value of the multipliers. No new constraint binds in the process. The argument for this is identical as the one in point 2 , except for $\mathrm{IC}_{h L, l H}$, which follows because $x_{h H}^{F B}\left[W_{l H}\left(q_{h H}\right)-W_{h L}\left(q_{h H}\right)\right] \stackrel{U_{l L, h L}=U_{l L, l H}}{=} x_{h L} \Delta \theta_{1}-x_{l H} q_{l H} \Delta \theta_{2}<$ $x_{l H}\left[W_{l H}\left(q_{l H}\right)-W_{h L}\left(q_{l H}\right)\right]$ when $x_{l H}>x_{h L}$. [Solution 1.1.e]

(d) We have reached $x_{h H}=x_{h L}$. At this point, $\mathrm{IC}_{l L, h H}$ starts binding. The rest of the argument is as in point c above: There exists a value for $\lambda_{4}$ and $\lambda_{6}$ such that $V W_{l H}=$ $V W_{h H}=V W_{h L}$ and $U_{l L, l H}=U_{l L, h L}=U_{l L, h H}$ and $N\left(\alpha_{l H} x_{l H}+\alpha_{h L} x_{h L}+\alpha_{l L} x_{l L}^{F B}+\right.$ $\left.\alpha_{h H} x_{h H}\right)=1$. The solution is thus Solution 1.1.e.

Scenario 2: At $q_{h H}^{2}, U_{h L, h H}<U_{h L, l H}$, that is, $x_{h H}^{F B}\left[W_{l H}\left(q_{h H}^{2}\right)-W_{h L}\left(q_{h H}^{2}\right)\right]>x_{l H}^{F B}\left[W_{l H}(\bar{q})-\right.$ $\left.W_{h L}(\bar{q})\right]$.

In this case, $\mathrm{IC}_{h L, l H}$ becomes binding as we decrease $q_{h H}$. To reduce $h L$ and $l L$ 's rents further, one now needs to decrease $q_{l H}$ at the same time as $q_{h H}$ in such a way that $U_{h L, h H}=U_{h L, l H}$, i.e., $x_{h H}^{F B}\left[W_{l H}\left(q_{h H}\right)-W_{h L}\left(q_{h H}\right)\right]=x_{l H}^{F B}\left[W_{l H}\left(q_{l H}\right)-W_{h L}\left(q_{l H}\right)\right]$. (Note that this implies that $q_{l H}>q_{h H}$.) Formally, using (1) to (4) in Appendix A, we let $q_{l H}$ and $q_{h H}$ solve:

$$
\begin{aligned}
V W_{l H} & =\max _{q_{l H}}\left\{W_{l H}\left(q_{l H}\right)+\frac{\lambda_{2}^{*}}{\alpha_{l H}}\left[W_{l H}\left(q_{l H}\right)-W_{h L}\left(q_{l H}\right)\right]\right\} \\
V W_{h H} & =\max _{q_{h H}}\left\{W_{h H}\left(q_{h H}\right)-\frac{\alpha_{l H}+\lambda_{2}^{*}}{\alpha_{h H}} \Delta \theta_{1}-\frac{\left(\alpha_{h L}+\alpha_{l L}-\lambda_{2}^{*}\right)}{\alpha_{h H}} \Delta \theta_{2} q_{h H}\right\}
\end{aligned}
$$

for the value of $\lambda_{2}^{*} \in\left(0, \alpha_{h L}+\alpha_{l L}\right)$ such that $x_{h H}^{F B}\left[W_{l H}\left(q_{h H}\right)-W_{h L}\left(q_{h H}\right)\right]=x_{l H}^{F B}\left[W_{l H}\left(q_{l H}\right)-\right.$ $\left.W_{h L}\left(q_{l H}\right)\right]\left(\lambda_{2}\right.$ is the multiplier on $\left.\mathrm{IC}_{h L, l H}\right)$. Such value for $\lambda_{2}$ always exists. When $\lambda_{2}^{*}=0$, $q_{l H}=\bar{q}$ and $q_{h H}=q_{h H}^{2}$ so that $x_{h H}^{F B}\left[W_{l H}\left(q_{h H}^{2}\right)-W_{h L}\left(q_{h H}^{2}\right)\right]>x_{l H}^{F B}\left[W_{l H}(\bar{q})-W_{h L}(\bar{q})\right]$ from the definition of scenario 2. When $\lambda_{2}^{*}=\alpha_{h L}+\alpha_{l L}, q_{l H}<q_{h H}=\bar{q}$ and $x_{h H}^{F B}\left[W_{l H}(\bar{q})-W_{h L}(\bar{q})\right]<$ $\left.x_{l H}^{F B}\left[W_{l H}\left(q_{l H}^{2}\right)-W_{h L}\left(q_{l H}^{2}\right)\right]\right)$.

Relative to the BOEM, only the rents of $h L$ and $l L$ have decreased. The IC constraint of $h L$ is taken care of by construction, and $U_{l L, h L} \geq U_{l L, l H}$ from Lemma $7(1)$. Hence, all IC constraints remain satisfied. 
We now optimize over the $x$ 's. Notice that $V W_{l H}=\max _{q_{l H}}\left\{W_{l H}\left(q_{l H}\right)+\frac{\lambda_{2}^{*}}{\alpha_{l H}}\left[W_{l H}\left(q_{l H}\right)-W_{h L}\left(q_{l H}\right)\right]\right\}>$ $W_{l H}(\bar{q})>V W_{h H}$. Hence, we need to consider three cases depending on the relative ranking of the virtual welfare associated with $h L$.

1. $V W_{h L} \geq V W_{l H}>V W_{h H}: W_{h L}(\underline{q})-\frac{\alpha_{l L}}{\alpha_{h L}} \Delta \theta_{1} \geq \max _{q_{l H}}\left\{W_{l H}\left(q_{l H}\right)+\frac{\lambda_{2}^{*}}{\alpha_{l H}}\left[W_{l H}\left(q_{l H}\right)-\right.\right.$ $\left.\left.W_{h L}\left(q_{l H}\right)\right]\right\} \quad$ [Solution 1.2.a]

The optimal probabilities are thus $x_{k}=x_{k}^{F B}$. The values of $q_{l H}$ and $q_{h H}$ are defined in (16) and $(17)$ and $\bar{q}>q_{l H}>q_{h H}>q_{h H}^{2}, q_{h L}=q_{l L}=\underline{q}$.

2. $V W_{l H}>V W_{h L} \geq V W_{h H}: \max _{q_{l H}}\left\{W_{l H}\left(q_{l H}\right)+\frac{\lambda_{2}^{*}}{\alpha_{l H}}\left[W_{l H}\left(q_{l H}\right)-W_{h L}\left(q_{l H}\right)\right]\right\}>W_{h L}(\underline{q})-$ $\frac{\alpha_{l L}}{\alpha_{h L}} \Delta \theta_{1} \geq W_{h H}\left(q_{h H}^{2}\right)-\frac{\alpha_{l H}}{\alpha_{h H}} \Delta \theta_{1}-\frac{\alpha_{h L}+\alpha_{l L}}{\alpha_{h H}} q_{h H}^{2} \Delta \theta_{2}$ (note that the condition is on $V W_{h H}$ evaluated at $\lambda_{2}=0$ ).

At the current value of $\lambda_{2}$, the buyer prefers to give the contract to $l H$ over $h L$. As we progressively increase $x_{l H}$ at the expense of $x_{h L}$, while keeping $x_{h H}^{F B}\left[W_{l H}\left(q_{h H}\right)-W_{h L}\left(q_{h H}\right)\right]=$ $x_{l H}\left[W_{l H}\left(q_{h L}\right)-W_{h L}\left(q_{h L}\right)\right]$, we decrease $\lambda_{2}$ (i.e. increase $q_{l H}$ and decrease $q_{h H}$ - from (16) and (17)). This decreases $V W_{l H}$ and increases $V W_{h H}$.

This process continues until the relative ordering of virtual welfares changes or the binding IC constraints change (at least of one these two events happen before we reach the feasibility constraint $\left.x_{l H}=x_{l H}^{\max }\right)$. Specifically, the two IC constraints we need to worry about are $\mathrm{IC}_{h L, l H}$ which stops binding when $\lambda_{2}=0$, and $\mathrm{IC}_{l L, l H}$ which starts binding when $x_{l H}=x_{h L}$. This yields three cases depending on which event happens first:

(a) $V W_{l H}=V W_{h L}$ first (note that given the assumption of this case, $V W_{h L} \geq V W_{h H}$ always): We have then reached the solution. At the solution, the probabilities of winning are: $x_{l L}=x_{l L}^{F B}>x_{h L}^{F B}>x_{h L}>x_{l H}>x_{l H}^{F B}>x_{h H}=x_{h H}^{F B}$ where $x_{l H}$ and $x_{h L}$ are defined implicitly by $x_{h H}^{F B}\left[W_{l H}\left(q_{h H}\right)-W_{h L}\left(q_{h H}\right)\right]=x_{l H}\left[W_{l H}\left(q_{l H}\right)-W_{h L}\left(q_{l H}\right)\right]$ for the values of $q_{h H}$ and $q_{l H}$ that solve (16) and (17) at the current value of $\lambda_{2}\left(q_{h H}<q_{l H}\right)$. The $x$ 's are optimized given the virtual welfares. The $q$ 's are optimized given the binding constraints and the value of $\lambda_{2}$. [Solution 1.2.b]

(b) $\lambda_{2}=0$ first. $\mathrm{IC}_{h L, l H}$ ceases to bind and $q_{h H}=q_{h H}^{2}$ and $q_{l H}=\bar{q}$. As $x_{l H}$ further increases and $x_{h L}$ decreases, the buyer increases his expected utility. None of the virtual welfares are affected in the process, and thus this continues until we either reach $x_{l H}=x_{l H}^{\max }$ or $\mathrm{IC}_{l L, l H}$ starts binding (this happens when $x_{h H}^{F B}\left[W_{l H}\left(q_{h H}^{2}\right)-W_{h L}\left(q_{h H}^{2}\right)\right]=x_{h L} \Delta \theta_{1}-$ $\left.x_{h L} \Delta \theta_{2} \bar{q}.\right)$. 
In the first case, we are as in Solution 1.1.b: $x_{l L}=x_{l L}^{F B}>x_{l H}=x_{l H}^{\max }>x_{h L}=x_{h L}^{\min }>$ $x_{h H}=x_{h H}^{F B}, q_{h H}=q_{h H}^{2}$ and $q_{l H}=\bar{q}$. The $x$ 's are optimized given that, by assumption, $V W_{h L} \geq V W_{h H}$.

In the second case, we are as in Solution 1.1c. Thus, $x_{l L}=x_{l L}^{F B}>x_{l H}^{\max } \geq x_{l H}>$ $x_{h L} \geq x_{h L}^{\min }>x_{h H}=x_{h H}^{F B}, q_{l L}=q_{h L}=\underline{q}$ and $q_{l H}$ and $q_{h H}$ defined by (8) and (9), $q_{l H}$, $q_{h H}<\bar{q}$.

(c) $x_{l H}=x_{h L}$ first. At this point, $\mathrm{IC}_{l L, l H}$ starts binding. Based on the expressions from (1), reworked using the equalities (2) to (4), the associated virtual welfares are given by:

$$
\begin{aligned}
V W_{l H} & =\max _{q_{l H}}\left\{W_{l H}\left(q_{l H}\right)-\frac{\left(\alpha_{h L}+\alpha_{l L}-\lambda_{3}\right)}{\alpha_{l H}} \Delta \theta_{2} q_{l H}+\frac{\alpha_{h L}+\lambda_{5}-\lambda_{3}}{\alpha_{l H}} \Delta \theta_{1}\right\} \\
V W_{h H} & =\max _{q_{h H}}\left\{W_{h H}\left(q_{h H}\right)-\frac{\lambda_{3}}{\alpha_{h H}} \Delta \theta_{2} q_{h H}-\frac{\alpha_{l H}+\alpha_{h L}+\alpha_{l L}-\lambda_{3}}{\alpha_{h H}} \Delta \theta_{1}\right\} \\
V W_{h L} & =W_{h L}(\underline{q})-\frac{\lambda_{5}}{\alpha_{h L}} \Delta \theta_{1}
\end{aligned}
$$

There exist values for $\lambda_{3}$ and $\lambda_{5}$ such that $(1) \bar{x}\left[W_{l H}\left(q_{l H}\right)-W_{h L}\left(q_{l H}\right)\right]=x_{h H}^{F B}\left[W_{l H}\left(q_{h H}\right)-\right.$ $\left.W_{h L}\left(q_{h H}\right)\right]$ and (2) $V W_{l H}=V W_{h L}$. To see this, note that the progressive adjustment of $x_{l H}$ until $x_{l H}=x_{h L}$ implies that there exists a value for $\lambda_{3}$ that satisfies condition (1). Once $\lambda_{3}$ is fixed, there is a value of $\lambda_{5}$ that ensures condition (2). Indeed for any feasible $\lambda_{3}$, when $\lambda_{5}=0$, the virtual welfare of $h L$ is greater. When $\lambda_{5}=\alpha_{l L}$ and $\lambda_{2}=\alpha_{h L}+\alpha_{l L}-\lambda_{3}$, this follows from the fact that we have assume that $V W_{l H}>V W_{h L}$ when $\mathrm{IC}_{l L, l H}$ becomes binding.

Note that $\lambda_{2}=\alpha_{h L}-\lambda_{3}+\lambda_{5}$. If the implied $\lambda_{2}$ is positive, this is the solution: $x_{l L}=$ $x_{l L}^{F B}>x_{h L}^{F B}>x_{h L}=\bar{x}=x_{l H}>x_{l H}^{F B}>x_{h H}=x_{h H}^{F B}$ and the $q$ 's solving (18) through (20) above for the values of $\lambda_{3}$ and $\lambda_{5}$ that satisfy conditions (1) and (2) (in particular, $\left.q_{l H}>q_{h H}\right)$. The $x$ 's are optimized given the virtual welfares: the buyer is indifferent between $l H$ and $h L$ and $V W_{l H}>V W_{h H}$ follows from the comparison between (18) and (19) when $q_{l H}>q_{h H}$. The $q$ 's are optimized given the binding constraints and the value of the multipliers. [Solution 1.2.c]

If the implied $\lambda_{2}$ is strictly negative, then $\mathrm{IC}_{h L, l H}$ ceases to bind at some point. We are then in the same situation as in Solution 1.1.c. At the solution, $x_{l L}=x_{l L}^{F B}>x_{l H}^{\max } \geq$ $x_{l H}>x_{h L} \geq x_{h L}^{\min }>x_{h H}=x_{h H}^{F B}, q_{l L}=q_{h L}=\underline{q}$ and $q_{l H}$ and $q_{h H}$ defined by (8) and (9), $q_{l H}, q_{h H}<\bar{q}$.

3. $V W_{l H}>V W_{h H}>V W_{h L}: W_{h L}(\underline{q})-\frac{\alpha_{l L}}{\alpha_{h L}} \Delta \theta_{1}<W_{h H}\left(q_{h H}^{2}\right)-\frac{\alpha_{l H}}{\alpha_{h H}} \Delta \theta_{1}-\frac{\alpha_{h L}+\alpha_{l L}}{\alpha_{h H}} q_{h H}^{2} \Delta \theta_{2}$ (note that the condition is on $V W_{h H}$ evaluated at $\lambda_{2}=0$ ). 
In this case, we ideally want to decrease $x_{h L}$, first to the benefit of $x_{l H}$ (then, possibly to the benefit of $\left.x_{h H}\right)$. Doing this while keeping $x_{h H}^{F B}\left[W_{l H}\left(q_{h H}\right)-W_{h L}\left(q_{h H}\right)\right]=x_{l H}\left[W_{l H}\left(q_{l H}\right)-\right.$ $W_{h L}\left(q_{l H}\right)$ ], requires that we decrease $\lambda_{2}$ (cf. (16) and (17)). This decreases $V W_{l H}$ and increases $V W_{h H}$, but given the condition on this case, the ordering of virtual welfares is not affected. Thus, this process continues until, either we reach $\lambda_{2}=0$ (and thus $\mathrm{IC}_{h L, l H}$ ceases to bind) or $x_{l H}=x_{h L}$ (and thus $\mathrm{IC}_{l L, l H}$ starts binding).

(a) We reach $x_{l H}=x_{h L}$ when $\lambda_{2}>0$ : This implies that $I C_{l L, l H}$ becomes binding in the process. Optimizing from now on with constraints $I C_{l H, h H}, I C_{l L, l H}, I C_{l L, h L}, I C_{h L, l H}$ and $I C_{h L, h H}$ binding requires that we keep $x_{l H}=x_{h L}$. The virtual welfares are given by (18), (19) and (20). Like in part 1 , scenario 2, case 2c, we proceed by first looking for values of $\lambda_{3}, \lambda_{5}$ and $q$ 's such that $(1) \bar{x}\left[W_{l H}\left(q_{l H}\right)-W_{h L}\left(q_{l H}\right)\right]=x_{h H}^{F B}\left[W_{l H}\left(q_{h H}\right)-\right.$ $\left.W_{h L}\left(q_{h H}\right)\right]$, i.e. $U_{l L, h L}=U_{l L, l H}$ and $U_{h L, h H}=U_{h L, h H}$ and (2) $V W_{l H}=V W_{h L}$.

If the implied $\lambda_{2}$ is positive, then this is the solution (solution 1.2.c) because condition (1) implies that $q_{l H}>q_{h H}$, which in turn ensures that $V W_{l H}=V W_{h L}>V W_{h H}$. The $x$ 's are optimized, and so are the $q$ 's.

If the implied $\lambda_{2}$ is negative, then we are as in part I, scenario 1, case 3: the binding constraints are $\mathrm{IC}_{l L, l H}, \mathrm{IC}_{l L, h L}, \mathrm{IC}_{l H, h H}$ and $\mathrm{IC}_{h L, h H}$. This leads to solutions 1.1.c, 1.1.d or 1.1.e.

(b) We reach $\lambda_{2}=0$ when $x_{l H} \leq x_{h L}$. We can continue to increase $x_{l H}$ at the expense of $x_{h L}$, and afterwards if necessary increase $x_{h H}$ at the expense of $x_{h L}$ until $I C_{l L, l H}$ starts binding. ( $I C_{h L, l H}$ no longer binds because increasing $x_{l H}$ beyond $x_{h L}$ means that $\left.x_{h H}\left[W_{l H}\left(q_{h H}\right)-W_{h L}\left(q_{h H}\right)\right]<x_{l H}\left[W_{l H}\left(q_{l H}\right)-W_{h L}\left(q_{l H}\right)\right]\right)$. The case then reduces to part 1 , scenario 1 , case 3 , implying one of solutions 1.1.c, 1.1.d or 1.1.e apply.

Proof of part II of Theorem 1: $W_{l H}(\bar{q})-W_{h L}(\bar{q})<0$ i.e. $\Delta \theta_{1}<\bar{q} \Delta \theta_{2}$

The binding constraints in the buyer-optimal efficient mechanism are $\mathrm{IC}_{l H, h H}, \mathrm{IC}_{h L, l H}$ and $\mathrm{IC}_{l L, h L}$. The buyer's resulting expected utility is given by

$$
\begin{aligned}
& \alpha_{l H} x_{l H}\left[W_{l H}\left(q_{l H}\right)+\frac{\alpha_{h L}+\alpha_{l L}}{\alpha_{l H}} \Delta \theta_{1}-\frac{\alpha_{h L}+\alpha_{l L}}{\alpha_{l H}} q_{l H} \Delta \theta_{2}\right]+\alpha_{h H} x_{h H}\left[W_{h H}\left(q_{h H}\right)-\frac{\alpha_{l H}+\alpha_{h L}+\alpha_{l L}}{\alpha_{h H}} \Delta \theta_{1}\right] \\
& +\alpha_{h L} x_{h L}\left[W_{h L}\left(q_{h L}\right)-\frac{\alpha_{l L}}{\alpha_{h L}} \Delta \theta_{1}\right]+\alpha_{l L} x_{l L} W_{l L}\left(q_{l L}\right)
\end{aligned}
$$

Keeping the probabilities fixed at $x_{k}=x_{k}^{F B}$, optimizing the $q$ 's requires that $q_{l H}$ be set equal to

$$
q_{l H}^{2}=\arg \max \left\{W_{l H}\left(q_{l H}\right)+\frac{\alpha_{h L}+\alpha_{l L}}{\alpha_{l H}} \Delta \theta_{1}-\frac{\alpha_{h L}+\alpha_{l L}}{\alpha_{l H}} q_{l H} \Delta \theta_{2}\right\}
$$


This reduces the informational rents of $h L$ and $l L$. By Lemma $7(1)$, we know that $U_{l L, h L}>U_{l L, l H}$ as long as $U_{h L, l H} \geq U_{h L, h H}$. Hence, we need to consider only two scenarios, depending on whether $I C_{h L, h H}$ binds at $q_{L H}^{2}$ :

Scenario 1: At $q_{l H}^{2}, U_{h L, l H} \geq U_{h L, h H}$, i.e., $x_{l H}^{F B}\left[W_{l H}\left(q_{l H}^{2}\right)-W_{h L}\left(q_{l H}^{2}\right)\right] \leq x_{h H}^{F B}\left[W_{l H}(\bar{q})-W_{h L}(\bar{q})\right]$ In this case, all IC constraints remain satisfied as we decrease $q_{l H}$ to $q_{l H}^{2}$. Note that $W_{l H}\left(q_{l H}^{2}\right)-$ $W_{h L}\left(q_{l H}^{2}\right) \equiv \Delta \theta_{1}-\Delta \theta_{2} q_{l H}^{2}<0$. We now consider the optimization of the probabilities of winning. From (21), the virtual welfare associated with $l L$ is the largest. This leaves four cases depending on the relative ranking of $l H, h H$ and $h L$ :

1. $V W_{h L} \geq V W_{l H} \geq V W_{h H}:\left[W_{h L}(\underline{q})-\frac{\alpha_{l L}}{\alpha_{h L}} \Delta \theta_{1}\right] \geq\left[W_{l H}\left(q_{l H}^{2}\right)+\frac{\alpha_{h L}+\alpha_{l L}}{\alpha_{l H}} \Delta \theta_{1}-\frac{\alpha_{h L}+\alpha_{l L}}{\alpha_{l H}} q_{l H}^{2} \Delta \theta_{2}\right] \geq$ $\left[W_{h H}(\bar{q})-\frac{\alpha_{l H}+\alpha_{h L}+\alpha_{l L}}{\alpha_{h H}} \Delta \theta_{1}\right] \quad$ [Solution 2.1.a]

The optimal probabilities of winning are $x_{k}=x_{k}^{F B}$ since the ranking of the virtual welfares corresponds to the ranking of the first best welfares. All IC constraints are satisfied. The $x$ 's and $q$ 's are optimized given the binding constraints.

2. $V W_{l H}>V W_{h H} \geq V W_{h L}:\left[W_{l H}\left(q_{l H}^{2}\right)+\frac{\alpha_{h L}+\alpha_{l L}}{\alpha_{l H}} \Delta \theta_{1}-\frac{\alpha_{h L}+\alpha_{l L}}{\alpha_{l H}} q_{l H}^{2} \Delta \theta_{2}\right]>W_{h H}(\bar{q})-\frac{\alpha_{l H}+\alpha_{h L}+\alpha_{l L}}{\alpha_{h H}} \Delta \theta_{1} \geq$ $\left[W_{h L}(\underline{q})-\frac{\alpha_{l L}}{\alpha_{h L}} \Delta \theta_{1}\right] ;$ or

$V W_{l H}>V W_{h L} \geq V W_{h H}:\left[W_{l H}\left(q_{l H}^{2}\right)+\frac{\alpha_{h L}+\alpha_{l L}}{\alpha_{l H}} \Delta \theta_{1}-\frac{\alpha_{h L}+\alpha_{l L}}{\alpha_{l H}} q_{l H}^{2} \Delta \theta_{2}\right]>\left[W_{h L}(\underline{q})-\frac{\alpha_{l L}}{\alpha_{h L}} \Delta \theta_{1}\right] \geq$ $W_{h H}\left(q_{h H}\right)-\frac{\alpha_{l H}+\alpha_{h L}+\alpha_{l L}}{\alpha_{h H}} \Delta \theta_{1}$

The buyer would like to increase $x_{l H}$ at the expense of $x_{h L}$. Doing this does not affect the supplier $h L$ 's IC constraint: $U_{h L, l H} \geq U_{h L, h H}$ corresponds to $x_{l H}\left[W_{l H}\left(q_{l H}^{2}\right)-W_{h L}\left(q_{l H}^{2}\right)\right] \leq$ $x_{h H}^{F B}\left[W_{l H}(\bar{q})-W_{h L}(\bar{q})\right]$ and $W_{l H}\left(q_{l H}^{2}\right)-W_{h L}\left(q_{l H}^{2}\right)<0$. Moreover, as long as $x_{h L}>x_{l H}$, the change in $x_{l H}$ does not affect $l L$ 's IC constraint either (Lemma $7(1)$ ). Thus, changing $x_{l H}$ does not initially affect the virtual welfares.

When we reach $x_{l H}=x_{h L}=\bar{x}, \mathrm{IC}_{l L, l H}$ starts binding since $U_{l L, h L}=x_{h L} \Delta \theta_{1}-x_{l H} \Delta \theta_{1}+$ $x_{l H} q_{l H}^{2} \Delta \theta_{2}+x_{h H} \Delta \theta_{1}$ and $U_{l L, l H}=x_{l H} q_{l H}^{2} \Delta \theta_{2}+x_{h H} \Delta \theta_{1}$. Define $\lambda_{5}^{*} \in\left(0, \alpha_{l L}\right)$, the value of $\lambda_{5}$ that equalizes the virtual welfares associated with $l H$ and $h L$ :

$$
W_{l H}\left(q_{l H}^{2}\right)+\frac{\left(\alpha_{h L}+\lambda_{5}^{*}\right)}{\alpha_{l H}} \Delta \theta_{1}-\frac{\alpha_{h L}+\alpha_{l L}}{\alpha_{l H}} \Delta \theta_{2} q_{l H}^{2}=W_{h L}(\underline{q})-\frac{\lambda_{5}^{*}}{\alpha_{h L}} \Delta \theta_{1}
$$

(from (1) to (4)). Such a value for $\lambda_{5}$ exists. When $\lambda_{5}=0$, the virtual welfare associated with $h L$ is larger. When $\lambda_{5}=\alpha_{l L}$, the virtual welfare of $l H$ is bigger by assumption. Note that this process does not affect the virtual welfare associated with $h H$, which remains unchanged.

(a) [Solution 2.1.b] If at $\lambda_{5}^{*}, V W_{l H}=V W_{h L}>V W_{h H}$, then the solution is $q_{l H}=q_{l H}^{2}$, $q_{h H}=\bar{q}$ and $q_{h L}=q_{l L}=\underline{q}$ and $x_{l L}=x_{l L}^{F B}, x_{h H}=x_{h H}^{F B}$, and $x_{l H}=x_{h L}=\bar{x}$. All IC 
constraints are satisfied. The $q$ 's and the $x$ 's are optimized given the binding constraints (in particular, the buyer is indifferent between $l H$ and $h L$, but strictly prefer these to $h H)$.

(b) If at $\lambda_{5}^{*}, V W_{l H}=V W_{h L}<V W_{h H}$, the buyer prefers $h H$ to $l H$ or $h L$. He increases his expected utility by raising $x_{h H}$ while keeping $U_{l L, l H}=U_{l L, h L}$, that is, $x_{l H}=x_{h L}$, and $\lambda_{5}=\lambda_{5}^{*}$. This process does not initially affect any of the virtual welfares until $\mathrm{IC}_{h L, h H}$ starts binding (this happens at $x_{h L}=x_{l H}>x_{h H}$ given that $q_{l H}=q_{l H}^{2}<q_{h H}=\bar{q}$ when $\left.U_{h L, h H} \leq U_{h L, l H}\right)$.

From then on, $\mathrm{IC}_{l H, h H}, \mathrm{IC}_{l L, l H}, \mathrm{IC}_{l L, h L}, \mathrm{IC}_{h L, l H}$ and $\mathrm{IC}_{h L, h H}$ are all binding. The expressions for the resulting virtual welfares are given by:

$$
\begin{aligned}
V W_{l H} & =\max _{q_{l H}}\left\{W_{l H}\left(q_{l H}\right)-\frac{\left(\alpha_{h L}+\alpha_{l L}-\lambda_{3}\right)}{\alpha_{l H}} \Delta \theta_{2} q_{l H}+\frac{\alpha_{h L}+\lambda_{5}-\lambda_{3}}{\alpha_{l H}} \Delta \theta_{1}\right\} \\
V W_{h H} & =\max _{q_{h H}}\left\{W_{h H}\left(q_{h H}\right)-\frac{\lambda_{3}}{\alpha_{h H}} \Delta \theta_{2} q_{h H}-\frac{\alpha_{l H}+\alpha_{h L}+\alpha_{l L}-\lambda_{3}}{\alpha_{h H}} \Delta \theta_{1}\right\} \\
V W_{h L} & =W_{h L}(\underline{q})-\frac{\lambda_{5}}{\alpha_{h L}} \Delta \theta_{1}
\end{aligned}
$$

The buyer increases his expected utility by continuing to increase $x_{h H}$ at the cost of $x_{h L}$ and $x_{l H}$, while satisfying: (1) $U_{l L, l H}=U_{l L, h L}\left(\right.$ thus $\left.x_{l H}=x_{h L}\right),(2) U_{h L, h H}=U_{h L, l H}$, that is $x_{l H}\left[W_{l H}\left(q_{l H}\right)-W_{h L}\left(q_{l H}\right)\right]=x_{h H}\left[W_{l H}\left(q_{h H}\right)-W_{h L}\left(q_{h H}\right)\right]$, and (3) $V W_{l H}=V W_{h L}$. This requires an increase in $\lambda_{3}$ and a decrease in $\lambda_{5}$, i.e. a rise in $q_{l H}$ and a decrease in $q_{h H}$ (nonetheless, $q_{l H}^{2}<q_{l H}<q_{h H}$ remains as long as $V W_{l H} \leq V W_{h H}$ as is apparent from $(24)$ and $(25)){ }^{3}$

This process stops when either $V W_{h H}=V W_{l H}=V W_{h L}$ or we hit a non negativity constraint for the multiplier $\lambda_{2}=\alpha_{h L}+\lambda_{5}-\lambda_{3}$.

i. [Solution 2.1.d] Suppose $V W_{h H}=V W_{l H}=V W_{h L}$ at a point where $\lambda_{2} \geq 0$. Then we have reached the solution. The $q$ 's are defined from (24) and (25) for the values of $\lambda_{3}$ and $\lambda_{5}$ that equalize the virtual welfares (note that this implies that $q_{l H}<q_{h H}$, so that, in turn, $U_{h L, h H}=U_{h L, l H}$ implies $x_{l H}>x_{h H}$ as required for incentive compatibility). The $x$ 's are such that $x_{l L}=x_{l L}^{F B}$, and $x_{l H}^{F B}>x_{l H}=$ $x_{h L}>x_{h H}>x_{h H}^{F B}$ with $N\left(\alpha_{l L} x_{l L}^{F B}+\alpha_{l H} x_{l H}+\alpha_{h L} x_{h L}+\alpha_{h H} x_{h H}\right)=1 .{ }^{4}$ All IC constraints are satisfied. The $q$ 's are optimized given the binding constraints. The

\footnotetext{
${ }^{3}$ Formally, we have four equations (the three constraints mentioned in the text, plus the feasibility constraint $\left.N\left(\alpha_{l L} x_{l L}^{F B}+\alpha_{l H} x_{l H}+\alpha_{h L} x_{h L}+\alpha_{h H} x_{h H}\right)=1\right)$ and five unknowns: $x_{h H}, x_{h L}, x_{l H}$ and $\lambda_{3}$ and $\lambda_{5}$ (the $q$ 's are determined on the basis of the $\lambda$ 's by (24) and (25)). Thus any value for $x_{h H}$ pins down the other variables.

${ }^{4}$ No other feasibility constraint for the probabilities of winning binds, except for the one-type constraint for $x_{l L}$.
} 
$x$ 's are optimized given the resulting virtual welfares (the buyer is indifferent among $l H, h L$ and $h H)$.

ii. [Solution 2.1.e] Suppose $\lambda_{2}$ reaches zero at a point where $V W_{h H}>V W_{l H}=$ $V W_{h L}$.

Let $\lambda_{5}^{* *}$, the value of $\lambda_{5}$ at this point. We also have $q_{l H}^{2}<q_{l H}<q_{h H}$ and $x_{l H}=$ $x_{h L}>x_{h H}$ at this point. The buyer further increases his utility by increasing $x_{h H}$ at the cost of $x_{l H}$ and $x_{h L}$, while keeping $U_{l L, l H}=U_{l L, h L}$ and $V W_{l H}=V W_{h L}$ (i.e. $\lambda_{5}=\lambda_{5}^{* *}$ and the $q$ 's are fixed at $\left.q_{l H}<q_{h H}\right) .{ }^{5}$ This process at first does not affect the virtual welfares (since $\lambda_{5}$ is fixed, we keep having $V W_{h H}>V W_{l H}=V W_{h L}$ ), until $\mathrm{IC}_{l L, h H}$ starts binding. ${ }^{6}$ At this stage we have:

$$
\begin{aligned}
U_{l L, l H} & =x_{h H} \Delta \theta_{1}+x_{l H} q_{l H} \Delta \theta_{2}=U_{l L, h H}=x_{h H} \Delta \theta_{1}+x_{h H} q_{h H} \Delta \theta_{2} \\
& =U_{l L, h L}=x_{h L} \Delta \theta_{1}+x_{h H} q_{h H} \Delta \theta_{2}
\end{aligned}
$$

thus $x_{h L}=x_{h H}<x_{l H}$. To keep increasing the buyer's welfare while satisfying all three constraints out of $l L$ requires that we keep $x_{h L}=x_{h H}$. Thus we increase both $x_{h L}$ and $x_{h H}$ at the expense of $x_{l H}$ (this will indeed increase the buyer's utility since $V W_{h H}>V W_{l H}=V W_{h L}$ ), and adjust the $q$ 's as needed, that is, we increase $q_{l H}$ and decrease $q_{h H}$. We do this until $V W_{l H}=V W_{h L}=V W_{h H}$. We have then reached the solution. At the solution, $q_{l H}<q_{h H}$ and $x_{l L}=x_{l L}^{F B}$, and $x_{l H}^{F B}>x_{l H}>x_{h L}=x_{h H}>x_{h H}^{F B}$ with $N\left(\alpha_{l L} x_{l L}^{F B}+\alpha_{l H} x_{l H}+\alpha_{h L} x_{h L}+\alpha_{h H} x_{h H}\right)=1$.

3. $V W_{h L}>V W_{h H}>V W_{l H}:\left[W_{h L}(\underline{q})-\frac{\alpha_{l L}}{\alpha_{h L}} \Delta \theta_{1}\right]>\left[W_{h H}(\bar{q})-\frac{\alpha_{l H}+\alpha_{h L}+\alpha_{l L}}{\alpha_{h H}} \Delta \theta_{1}\right]>\left[W_{l H}\left(q_{l H}^{2}\right)+\right.$ $\left.\frac{\alpha_{h L}+\alpha_{l L}}{\alpha_{l H}} \Delta \theta_{1}-\frac{\alpha_{h L}+\alpha_{l L}}{\alpha_{l H}} q_{l H}^{2} \Delta \theta_{2}\right] ;$ or

$V W_{h H}>V W_{h L}>V W_{l H}: W_{h H}(\bar{q})-\frac{\alpha_{l H}+\alpha_{h L}+\alpha_{l L}}{\alpha_{h H}} \Delta \theta_{1}>\left[W_{h L}(\underline{q})-\frac{\alpha_{l L}}{\alpha_{h L}} \Delta \theta_{1}\right]>\left[W_{l H}\left(q_{l H}^{2}\right)+\right.$ $\left.\frac{\alpha_{h L}+\alpha_{l L}}{\alpha_{l H}} \Delta \theta_{1}-\frac{\alpha_{h L}+\alpha_{l L}}{\alpha_{l H}} q_{l H}^{2} \Delta \theta_{2}\right]$

In this case, the buyer would like to increase $x_{h H}$ at the expense of $x_{l H}$. As we increase $x_{h H}$ and decrease $x_{l H}$, we reach a point where $x_{l H}\left[W_{l H}\left(q_{l H}^{2}\right)-W_{h L}\left(q_{l H}^{2}\right)\right]=x_{h H}\left[W_{l H}(\bar{q})-W_{h L}(\bar{q})\right]$, that is, $\mathrm{IC}_{h L, h H}$ starts binding.

A candidate solution is defined by the value of $\lambda_{2} \in\left(0, \alpha_{h L}+\alpha_{l L}\right)$ that equates $V W_{l H}$ and

\footnotetext{
${ }^{5}$ The exact way in which $x_{l H}$ and $x_{h L}$ are decreased is determined by $U_{l L, l H}=U_{l L, h L}$, i.e. $x_{l H} q_{l H} \Delta \theta_{2}+x_{h H} \Delta \theta_{1}=$ $x_{h L} \Delta \theta_{1}+x_{h H} q_{h H} \Delta \theta_{2}$ and the feasibility constraint $N\left(\alpha_{l L} x_{l L}^{F B}+\alpha_{l H} x_{l H}+\alpha_{h L} x_{h L}+\alpha_{h H} x_{h H}\right)=1$.

${ }^{6}$ This is the only constraint that can bind in the process. No new constraint can bind out of $l H$ since $U_{l H}=x_{h H} \Delta \theta_{1}$ increases and alternatives decrease. No new constraint can bind out of $h L$ because $\Delta \theta_{1}-\Delta \theta_{2} q_{h H}<0$ given that $q_{h H}>q_{l H}>q_{l H}^{2}$ and $V W_{h H} \geq V W_{l H}$.
} 
$V W_{h H}:$

$\max _{q_{l H}}\left\{W_{l H}\left(q_{l H}\right)+\frac{\lambda_{2}^{*}}{\alpha_{l H}} \Delta \theta_{1}-\frac{\lambda_{2}^{*}}{\alpha_{l H}} \Delta \theta_{2} q_{l H}\right\}=\max _{q_{h H}}\left\{W_{h H}\left(q_{h H}\right)-\frac{\alpha_{l H}+\lambda_{2}^{*}}{\alpha_{h H}} \Delta \theta_{1}-\frac{\alpha_{h L}+\alpha_{l L}-\lambda_{2}^{*}}{\alpha_{h H}} \Delta \theta_{2} q_{h H}\right\}$

(from (1) to (4)). Such value for $\lambda_{2}$ exists since the virtual welfare of $l H$ is larger than that of $h H$ at $\lambda_{2}=0$, and smaller at $\lambda_{2}=\alpha_{h L}+\alpha_{l L}$ by assumption. By inspection of (27), this happens at $\frac{\alpha_{h L}+\alpha_{l L}-\lambda_{2}^{*}}{\alpha_{h H}}<\frac{\lambda_{2}^{*}}{\alpha_{l H}}$ that is, the resulting $q$ 's are such that $q_{l H}^{2}<q_{l H}<q_{h H}$. Finally, we require that $U_{h L, l H}=U_{h L, h H}$, that is, $x_{l H}\left[W_{l H}\left(q_{l H}\right)-W_{h L}\left(q_{l H}\right)\right]=x_{h H}\left[W_{l H}\left(q_{h H}\right)-\right.$ $\left.W_{h L}\left(q_{h H}\right)\right]$ which implies that $x_{l H}>x_{h H}$ as required by incentive compatibility.

This process only affected $V W_{h L}$ and $V W_{h H}$. If $V W_{h L}>V W_{h H}=V W_{l H}$ at this point, then this is indeed the solution. The other variables are set such that $x_{l L}=x_{l L}^{F B}, x_{h L}=x_{h L}^{F B}$, and $q_{h L}=q_{l L}=\bar{q}$. The $q$ 's are optimized given the values of the multipliers and the binding constraints. The $x$ 's are optimized given the resulting virtual welfares. All IC constraints are satisfied $\left(\mathrm{IC}_{l L, l H}\right.$ satisfied given Lemma $\left.7(1)\right)$. [Solution 2.1.c]

If $V W_{h L}<V W_{h H}=V W_{l H}$, the buyer can further increase his expected utility by increasing $x_{h H}$ and $x_{l H}$ at the cost of $x_{h L}$. He does so while keeping $\lambda_{2}=\lambda_{2}^{*}$ so that $V W_{h H}=V W_{l H}$. The exact way in which $x_{h H}$ and $x_{l H}$ are increased is pinned down by $x_{l H}\left[W_{l H}\left(q_{l H}\right)-W_{h L}\left(q_{l H}\right)\right]=$ $x_{h H}\left[W_{l H}\left(q_{h H}\right)-W_{h L}\left(q_{h H}\right)\right]$. This process does not affect the virtual welfare, until $x_{h L}=x_{l H}$ at which point $\mathrm{IC}_{l L, l H}$ starts binding. We are now in a situation where $\mathrm{IC}_{l H, h H}, \mathrm{IC}_{l L, l H}$, $\mathrm{IC}_{l L, h L}, \mathrm{IC}_{h L, l H}$ and $\mathrm{IC}_{h L, h H}$ are all binding and $V W_{h L}<V W_{h H}=V W_{l H}$. From then on, the virtual welfares are those defined in $(24)-(26)$. Let $\lambda_{5}^{*}$ such that $V W_{l H}=V W_{h L}$. Since there is no change in $\lambda_{3}$, the $q$ 's are not affected $\left(q_{l H}<q_{h H}\right)$ and the $x$ 's implicitly defined by $x_{l H}=x_{h L}$ and $U_{h L, h H}=U_{h L, l H}$ are not affected either. Thus we are exactly in the same situation as in 2(b) above, and the proof thus proceeds along the same lines: we look for a solution where $\mathrm{IC}_{l H, h H}, \mathrm{IC}_{l L, l H}, \mathrm{IC}_{l L, h L}, \mathrm{IC}_{h L, l H}$ and $\mathrm{IC}_{h L, h H}$ are binding and $V W_{h L}=V W_{h H}=V W_{l H}$, or $\mathrm{IC}_{l H, h H}, \mathrm{IC}_{l L, l H}, \mathrm{IC}_{l L, h L}, \mathrm{IC}_{l L, h H}$ and $\mathrm{IC}_{h L, h H}$ are binding and $V W_{h L}=V W_{h H}=V W_{l H}$. [Solution 2.1.d or 2.1.e]

4. $V W_{h H}>V W_{l H}>V W_{h L}: W_{h H}(\bar{q})-\frac{\alpha_{l H}+\alpha_{h L}+\alpha_{l L}}{\alpha_{h H}} \Delta \theta_{1}>\left[W_{l H}\left(q_{l H}^{2}\right)+\frac{\alpha_{h L}+\alpha_{l L}}{\alpha_{l H}} \Delta \theta_{1}-\right.$ $\left.\frac{\alpha_{h L}+\alpha_{l L}}{\alpha_{l H}} q_{l H}^{2} \Delta \theta_{2}\right]>\left[W_{h L}(\underline{q})-\frac{\alpha_{l L}}{\alpha_{h L}} \Delta \theta_{1}\right]$

Given the ordering of virtual welfares, the buyer is first tempted to increase $x_{h H}$ at the expense of $x_{h L} \cdot{ }^{7}$ Two things can happen in the process: (1) $\mathrm{IC}_{l L, l H}$ starts binding (this happens at $x_{l H}^{F B}=x_{h L}$ because $U_{l L, l H}=x_{l H} \Delta \theta_{2} q_{l H}^{2}+x_{h H} \Delta \theta_{1}$ and $U_{l L, h L}=x_{h L} \Delta \theta_{1}-$ $\left.x_{l H} \Delta \theta_{1}+x_{l H} \Delta \theta_{2} q_{l H}^{2}+x_{h H} \Delta \theta_{1}\right)$, (2) $\mathrm{IC}_{h L, h H}$ starts binding (this happens at a point where

\footnotetext{
${ }^{7}$ That is, keeping the equality $N\left(\alpha_{l L} x_{l L}^{F B}+\alpha_{l H} x_{l H}^{F B}+\alpha_{h L} x_{h L}+\alpha_{h H} x_{h H}\right)=1$.
} 
$x_{h H}<x_{l H}^{F B}$ since $x_{h H}\left[W_{l H}(\bar{q})-W_{h L}(\bar{q})\right]=x_{l H}^{F B}\left[W_{l H}\left(q_{l H}^{2}\right)-W_{h L}\left(q_{l H}^{2}\right)\right]$ at that point, and $W_{l H}\left(q_{l H}^{2}\right)-W_{h L}\left(q_{l H}^{2}\right)<0$ from the definition of scenario 1$)$. We examine each case in turn.

(a) $I C_{l L, l H}$ binds first $\left(x_{l H}^{F B}=x_{h L}\right)$

Let $\lambda_{5}^{*}$, the value of $\lambda_{5}$ that equalizes $V W_{l H}$ and $V W_{h L}$. This was defined in (23). We now have $V W_{h H}>V W_{l H}=V W_{h L}$. Thus the buyer can increase his welfare by increasing $x_{h H}$. The rest of the solution is as described in 2(b) above. [Solution 2.1.d or Solution 2.1.e].

(b) $\mathrm{IC}_{h L, h H}$ binds first:

This happens at $x_{h L}>x_{l H}^{F B}>x_{h H}$ (the first inequality comes from the fact that $\mathrm{IC}_{h L, h H}$ binds first; the second inequality comes from the fact that $q_{l H}<q_{h H}=\bar{q}$ at the point where $\mathrm{IC}_{h L, h H}$ starts binding). Increasing further $x_{h H}$ at the expense of $x_{h L}$, while keeping $x_{l H}^{F B}\left[W_{l H}\left(q_{l H}\right)-W_{h L}\left(q_{l H}\right)\right]=x_{h H}\left[W_{l H}\left(q_{h H}\right)-W_{h L}\left(q_{h H}\right)\right]$ requires that we decrease $q_{h H}$ and increase $q_{l H}$. This corresponds to a rise in $\lambda_{3}$, a decrease in $V W_{h H}$ and an increase in $V W_{l H}$. This process stops when either $V W_{l H}=V W_{h H}$ or $x_{l H}=x_{h L}$ whichever comes first (note at this stage $x_{l H}=x_{h L}>x_{h H}$ and $I C_{l L, l H}$ starts binding). If $V W_{l H}=V W_{h H}$ first, we can continue to increase the buyer's utility by decreasing $x_{h L}$, this time to the benefit of both $l H$ and $h H$ while keeping $V W_{l H}=V W_{h H}$ and $U_{h L, h H}=U_{h L, l H}$ (note that this implies $q_{l H}<q_{h H}$ and $x_{h L}>x_{h H}$ ). This process continues until $x_{h L}=x_{l H}$ at which point $I C_{l L, l H}$ starts binding.

Thus, in both events, we reach a point where $\mathrm{IC}_{l H, h H}, \mathrm{IC}_{l L, l H}, \mathrm{IC}_{l L, h L}, \mathrm{IC}_{h L, l H}$ and $\mathrm{IC}_{h L, h H}$ are all binding. From then on, the virtual welfares are those defined in (24) (26). Let $\lambda_{5}^{*}$ such that $V W_{l H}=V W_{h L}$. Since there is no change in $\lambda_{3}$, the $q$ 's are not affected $\left(q_{l H}<q_{h H}\right)$ and the $x$ 's implicitly defined by $x_{l H}=x_{h L}$ and $U_{h L, h H}=U_{h L, l H}$ are not affected either. Thus we are exactly in the same situation as in 2(b) above, and the proof thus proceeds along the same lines: we look for a solution where $\mathrm{IC}_{l H, h H}$, $\mathrm{IC}_{l L, l H}, \mathrm{IC}_{l L, h L}, \mathrm{IC}_{h L, l H}$ and $\mathrm{IC}_{h L, h H}$ are binding and $V W_{h L}=V W_{h H}=V W_{l H}$, or $\mathrm{IC}_{l H, h H}, \mathrm{IC}_{l L, l H}, \mathrm{IC}_{l L, h L}, \mathrm{IC}_{l L, h H}$ and $\mathrm{IC}_{h L, h H}$ are binding and $V W_{h L}=V W_{h H}=$ $V W_{l H}$. [Solution 2.1.d or 2.1.e]

Scenario 2: At $q_{l H}^{2}, U_{h L, h H}>U_{h L, l H}$ that is, $x_{l H}^{F B}\left[W_{l H}\left(q_{l H}^{2}\right)-W_{h L}\left(q_{l H}^{2}\right)\right]>x_{h H}^{F B}\left[W_{l H}(\bar{q})-W_{h L}(\bar{q})\right]$ In this case, $\mathrm{IC}_{h L, h H}$ becomes binding as we decrease $q_{l H}$ towards $q_{l H}^{2}$. To decrease the rents of $h L$ and $l L$, we now need to decrease $q_{l H}$ and $q_{h H}$, holding $U_{h L, h H}=U_{h L, l H}$. The optimal $q$ 's are 
defined by:

$$
\begin{aligned}
q_{l H}^{*} & =\arg \max _{q_{l H}}\left\{W_{l H}\left(q_{l H}\right)+\frac{\lambda_{2}^{*}}{\alpha_{l H}} \Delta \theta_{1}-\frac{\lambda_{2}^{*}}{\alpha_{l H}} \Delta \theta_{2} q_{l H}\right\} \\
q_{h H}^{*} & =\arg \max _{q_{h H}}\left\{W_{h H}\left(q_{h H}\right)-\frac{\alpha_{l H}+\lambda_{2}^{*}}{\alpha_{h H}} \Delta \theta_{1}-\frac{\alpha_{h L}+\alpha_{l L}-\lambda_{2}^{*}}{\alpha_{h H}} \Delta \theta_{2} q_{h H}\right\}
\end{aligned}
$$

where $\lambda_{2}^{*} \in\left(0, \alpha_{h L}+\alpha_{l L}\right)$ is chosen such that $x_{l H}^{F B}\left[W_{l H}\left(q_{l H}^{*}\right)-W_{h L}\left(q_{l H}^{*}\right)\right]=x_{h H}^{F B}\left[W_{l H}\left(q_{h H}^{*}\right)-\right.$ $\left.W_{h L}\left(q_{h H}^{*}\right)\right]$. Note that the sign of $W_{l H}\left(q_{l H}^{*}\right)-W_{h L}\left(q_{l H}^{*}\right)=\Delta \theta_{1}-\Delta \theta_{2} q_{l H}^{*}$ is not pinned down a priori so that $q_{l H}$ and $q_{h H}$ cannot be ranked. No other new constraint binds in the process (Lemma 7(1)).

We now consider the optimization of the probabilities of winning. We need to consider five cases:

1. $V W_{h L} \geq V W_{l H} \geq V W_{h H}: W_{h L}(\underline{q})-\frac{\alpha_{l L}}{\alpha_{h L}} \Delta \theta_{1} \geq W_{l H}\left(q_{l H}^{*}\right)+\frac{\lambda_{2}^{*}}{\alpha_{l H}} \Delta \theta_{1}-\frac{\lambda_{2}^{*}}{\alpha_{l H}} \Delta \theta_{2} q_{l H}^{*} \geq$ $W_{h H}\left(q_{h H}^{*}\right)-\frac{\alpha_{l H}+\lambda_{2}^{*}}{\alpha_{h H}} \Delta \theta_{1}-\frac{\alpha_{h L}+\alpha_{l L}-\lambda_{2}^{*}}{\alpha_{h H}} \Delta \theta_{2} q_{h H}^{*}$.

The optimal probabilities of winning are $x_{k}=x_{k}^{F B}$. This corresponds to Solution 1.2.a except that $q_{l H}$ and $q_{h H}$ cannot be ranked a priori.

2. $V W_{l H}>V W_{h L} \geq V W_{h H}: W_{l H}\left(q_{l H}^{*}\right)+\frac{\lambda_{2}^{*}}{\alpha_{l H}} \Delta \theta_{1}-\frac{\lambda_{2}^{*}}{\alpha_{l H}} \Delta \theta_{2} q_{l H}^{*}>W_{h L}(\underline{q})-\frac{\alpha_{l L}}{\alpha_{h L}} \Delta \theta_{1} \geq$ $W_{h H}\left(q_{h H}^{*}\right)-\frac{\alpha_{l H}+\lambda_{2}^{*}}{\alpha_{h H}} \Delta \theta_{1}-\frac{\alpha_{h L}+\alpha_{l L}-\lambda_{2}}{\alpha_{h H}} \Delta \theta_{2} q_{h H}^{*}$

$V W_{l H}>V W_{h H}>V W_{h L}: W_{l H}\left(q_{l H}^{*}\right)+\frac{\lambda_{2}^{*}}{\alpha_{l H}} \Delta \theta_{1}-\frac{\lambda_{2}^{*}}{\alpha_{l H}} \Delta \theta_{2} q_{l H}^{*}>W_{h H}\left(q_{h H}^{*}\right)-\frac{\alpha_{l H}+\lambda_{2}^{*}}{\alpha_{h H}} \Delta \theta_{1}-$ $\frac{\alpha_{h L}+\alpha_{l L}-\lambda_{2}^{*}}{\alpha_{h H}} \Delta \theta_{2} q_{h H}^{*}>W_{h L}(\underline{q})-\frac{\alpha_{l L}}{\alpha_{h L}} \Delta \theta_{1}$

The buyer would like to increase $x_{l H}$ at the expense of $x_{h L}$. Doing this while keeping $U_{h L, h H}=$ $U_{h L, l H}$ requires that we adjust the $q$ 's and thus $\lambda_{2}$. Specifically, if $\Delta \theta_{1}-\Delta \theta_{2} q_{l H}^{*}>0$, we need to decrease $\lambda_{2}$, otherwise, we need to increase it. In both cases, $V W_{l H}$ goes down and $V W_{h H}$ goes up. This process continues until either a new IC constraint binds or the relative ranking of the virtual welfare changes. Since $x_{l H}>x_{l H}^{F B}>x_{h H}$, the only IC constraint to worry about is $\mathrm{IC}_{l L, l H}$. This gives us three cases to consider depending on which event happens first:

(a) $V W_{l H}=V W_{h L} \geq V W_{h H}$ : We have reached the solution: $x_{l L}=x_{l L}^{F B}, x_{h H}=x_{h H}^{F B}$ and $x_{h L}^{F B}>x_{h L}>x_{l H}>x_{l H}^{F B}$ with $N\left(\alpha_{l L} x_{l L}^{F B}+\alpha_{l H} x_{l H}+\alpha_{h L} x_{h L}\right)=1-\alpha_{h H}^{N}, q_{l L}=q_{h L}=\underline{q}$ and $q_{l H}$ and $q_{h H}$ determined by the value of $\lambda_{2}$ that equates $V W_{h H}=V W_{l H}$. This corresponds to Solution 1.2.b.

(b) $V W_{l H}=V W_{h H}>V W_{h L}$ : Note that this means that $q_{l H}<q_{h H}$ and $\Delta \theta_{1}-\Delta \theta_{2} q_{l H}<0$ since $x_{l H}\left[W_{l H}\left(q_{l H}\right)-W_{h L}\left(q_{l H}\right)\right]=x_{h H}^{F B}\left[W_{l H}\left(q_{l H}\right)-W_{h L}\left(q_{l H}\right)\right]$. The buyer continues to increase his expected utility by decreasing $x_{h L}$, this time, to the benefit of both $x_{l H}$ and $x_{h H}$, doing so while keeping $V W_{l H}=V W_{h H}$ and $U_{h L, l L}=U_{h L, l H}$. Thus $\lambda_{2}$ is fixed and 
so are $q_{l H}$ and $q_{h H}$. Therefore $x_{l H}>x_{h H}$. This process continues until $x_{h L}=x_{l H}$ at which point $\mathrm{IC}_{l L, l H}$ starts binding. From then on, the virtual welfares are those defined in (24) - (26). (note that $\lambda_{2}=\alpha_{h L}+\lambda_{5}-\lambda_{3}$ ). Let $\lambda_{5}^{*}$ such that $V W_{l H}=V W_{h L}$. Since there is no change in $\lambda_{3}$, the $q$ 's are not affected $\left(q_{l H}<q_{h H}\right)$ and the $x$ 's implicitly defined by $x_{l H}=x_{h L}$ and $U_{h L, h H}=U_{h L, l H}$ are not affected either. Thus we are exactly in the same situation as in scenario 1, 2(b) above $\left(V W_{h H}>V W_{l H}=V W_{h L}\right)$, and the proof thus proceeds along the same lines. [Solution 2.1.d or 2.1.e]

(c) $x_{h L}=x_{l H}$, i.e. $\mathrm{IC}_{l L, l H}$ starts binding. From then on, $\mathrm{IC}_{l H, h H}, \mathrm{IC}_{l L, l H}, \mathrm{IC}_{l L, h L}, \mathrm{IC}_{h L, l H}$ and $\mathrm{IC}_{h L, h H}$ are all binding. The virtual welfares are those defined in (24) - (26). (note that $\left.\lambda_{2}=\alpha_{h L}+\lambda_{5}-\lambda_{3}\right)$. Let $\lambda_{5}^{*}$ such that $V W_{l H}=V W_{h L}$. Since there is no change in $\lambda_{3}$, the $q$ 's are not affected and the $x$ 's implicitly defined by $x_{l H}=x_{h L}$ and $U_{h L, h H}=U_{h L, l H}$ are not affected either. If $V W_{l H}=V W_{h L}>V W_{h H}$, we have reached the solution: $x_{l L}=x_{l L}^{F B}, x_{h L}^{F B}>x_{l H}=x_{h L}=\bar{x}>x_{l H}^{F B}, x_{h H}=x_{h H}^{F B}, q_{l H}, q_{h H}<\bar{q}$ and $q_{l L}=q_{h L}=\bar{q}$. All IC constraints are satisfied and the $q$ 's and $x$ 's are optimal given the resulting virtual welfares. [Solution 1.2.c]

If $V W_{l H}=V W_{h L}<V W_{h H}$, we can conclude that $q_{l H}<q_{h H}$ and $\Delta \theta_{1}-\Delta \theta_{2} q_{l H}<0$ since $x_{l H}\left[W_{l H}\left(q_{l H}\right)-W_{h L}\left(q_{l H}\right)\right]=x_{h H}^{F B}\left[W_{l H}\left(q_{l H}\right)-W_{h L}\left(q_{l H}\right)\right]$. We are thus in the same situation as in scenario 1, 2(b) above. [Solution 2.1.d or 2.1.e]

3. $V W_{h L}>V W_{h H}>V W_{l H}: W_{h L}(\underline{q})-\frac{\alpha_{l L}}{\alpha_{h L}} \Delta \theta_{1}>W_{h H}\left(q_{h H}^{*}\right)-\frac{\alpha_{l H}+\lambda_{2}^{*}}{\alpha_{h H}} \Delta \theta_{1}-\frac{\alpha_{h L}+\alpha_{l L}-\lambda_{2}^{*}}{\alpha_{h H}} \Delta \theta_{2} q_{h H}^{*}>$ $W_{l H}\left(q_{l H}^{*}\right)+\frac{\lambda_{2}^{*}}{\alpha_{l H}} \Delta \theta_{1}-\frac{\lambda_{2}^{*}}{\alpha_{l H}} \Delta \theta_{2} q_{l H}^{*}$.

(Note that this implies $q_{l H}^{*}<q_{h H}^{*}$ and $\Delta \theta_{1}-\Delta \theta_{2} q_{l H}^{*}<0$ given that $x_{l H}^{F B}\left[W_{l H}\left(q_{l H}^{*}\right)-W_{h L}\left(q_{l H}^{*}\right)\right]$ $\left.=x_{h H}^{F B}\left[W_{l H}\left(q_{h H}^{*}\right)-W_{h L}\left(q_{h H}^{*}\right)\right]\right)$. The buyer wants to increase $x_{h H}$ at the expense of $x_{l H}$. This requires adjusting $\lambda_{2}$ to maintain the equality $x_{l H}\left[W_{l H}\left(q_{l H}\right)-W_{h L}\left(q_{l H}\right)\right]=x_{h H}\left[W_{l H}\left(q_{h H}\right)-\right.$ $\left.W_{h L}\left(q_{h H}\right)\right]$. Specifically, $\lambda_{2}$ decreases, $q_{l H}$ increases and $q_{h H}$ decreases, until $V W_{h H}=V W_{l H}$. This occurs at $x_{l H}>x_{h H}$. Indeed, at $x_{l H}=x_{h H}, q_{l H}=q_{h H}$ thus $\frac{\alpha_{h L}+\alpha_{l L}-\lambda_{2}}{\alpha_{h H}} \Delta \theta_{2} q_{h H}=$ $\frac{\lambda_{2}}{\alpha_{l H}} \Delta \theta_{2} q_{l H}$ implying that $V W_{h H}<V W_{l H}$. The solution is thus $x_{l L}=x_{l L}^{F B}, x_{h L}=x_{h L}^{F B}$ and $x_{l H}^{F B}>x_{l H}>x_{h H}>x_{h H}^{F B}$ and $q_{l H}<q_{l H}<q_{h H}<\bar{q}$. This corresponds to solution 2.1.c

4. $V W_{h H} \geq V W_{l H} \geq V W_{h L}: W_{h H}\left(q_{h H}^{*}\right)-\frac{\alpha_{l H}+\lambda_{2}^{*}}{\alpha_{h H}} \Delta \theta_{1}-\frac{\alpha_{h L}+\alpha_{l L}-\lambda_{2}^{*}}{\alpha_{h H}} \Delta \theta_{2} q_{h H}^{*} \geq W_{l H}\left(q_{l H}^{*}\right)+$ $\frac{\lambda_{2}^{*}}{\alpha_{l H}} \Delta \theta_{1}-\frac{\lambda_{2}^{*}}{\alpha_{l H}} \Delta \theta_{2} q_{l H}^{*} \geq W_{h L}(\underline{q})-\frac{\alpha_{l L}}{\alpha_{h L}} \Delta \theta_{1}$

Note that this implies that $q_{l H}^{*}<q_{h H}^{*}$ and $\Delta \theta_{1}-\Delta \theta_{2} q_{l H}^{*}<0$. Define $\lambda_{2}^{* *} \in\left(0, \lambda_{2}^{*}\right)$ such that

$\max _{q_{h H}}\left\{W_{h H}\left(q_{h H}\right)-\frac{\alpha_{l H}+\lambda_{2}^{* *}}{\alpha_{h H}} \Delta \theta_{1}-\frac{\alpha_{h L}+\alpha_{l L}-\lambda_{2}^{* *}}{\alpha_{h H}} \Delta \theta_{2} q_{h H}\right\}=\max _{q_{l H}}\left\{W_{l H}\left(q_{l H}\right)+\frac{\lambda_{2}^{* *}}{\alpha_{l H}} \Delta \theta_{1}-\frac{\lambda_{2}^{* *}}{\alpha_{l H}} \Delta \theta_{2} q_{l H}\right\}$ 
This implies $q_{l H}^{*}<q_{l H}<q_{h H}<q_{h H}^{*}$ and $V W_{l H}=V W_{h H}>V W_{h L}$.

$>$ From there, the buyer can increase his expected utility by increasing $x_{h H}$ and $x_{l H}$ at the cost of $x_{h L}$. He does so while keeping $\lambda_{2}=\lambda_{2}^{* *}$ so that $V W_{h H}=V W_{l H}$. The exact way in which $x_{h H}$ and $x_{l H}$ are increased is pinned down by $x_{l H}\left[W_{l H}\left(q_{l H}\right)-W_{h L}\left(q_{l H}\right)\right]=x_{h H}\left[W_{l H}\left(q_{h H}\right)-\right.$ $\left.W_{h L}\left(q_{h H}\right)\right]$. This process does not affect the virtual welfare, until $x_{h L}=x_{l H}$ at which point $\mathrm{IC}_{l L, l H}$ starts binding. We are now in a situation where $\mathrm{IC}_{l H, h H}, \mathrm{IC}_{l L, l H}, \mathrm{IC}_{l L, h L}, \mathrm{IC}_{h L, l H}$ and $\mathrm{IC}_{h L, h H}$ are all binding and $V W_{h L}<V W_{h H}=V W_{l H}$. From then on, the virtual welfares are those defined in (24) - (26) (note that $\lambda_{2}=\alpha_{h L}+\lambda_{5}-\lambda_{3}$ ). Let $\lambda_{5}^{*}$ such that $V W_{l H}=V W_{h L}$. Since there is no change in $\lambda_{3}$, the $q$ 's are not affected $\left(q_{l H}<q_{h H}\right)$ and the $x$ 's implicitly defined by $x_{l H}=x_{h L}$ and $U_{h L, h H}=U_{h L, l H}$ are not affected either. Thus we are exactly in the same situation as in 2(b) above. [Solution 2.1.d or 2.1.e]

5. $V W_{h H}>V W_{h L}>V W_{l H}: W_{h H}\left(q_{h H}^{*}\right)-\frac{\alpha_{l H}+\lambda_{2}^{*}}{\alpha_{h H}} \Delta \theta_{1}-\frac{\alpha_{h L}+\alpha_{l L}-\lambda_{2}^{*}}{\alpha_{h H}} \Delta \theta_{2} q_{h H}^{*}>W_{h L}(\underline{q})-$ $\frac{\alpha_{l L}}{\alpha_{h L}} \Delta \theta_{1}>W_{l H}\left(q_{l H}^{*}\right)+\frac{\lambda_{2}^{*}}{\alpha_{l H}} \Delta \theta_{1}-\frac{\lambda_{2}^{*}}{\alpha_{l H}} \Delta \theta_{2} q_{l H}^{*}$

We are again in a situation where $q_{l H}^{*}<q_{h H}^{*}$ and $\Delta \theta_{1}-\Delta \theta_{2} q_{h H}^{*}<0$. The buyer would like to increase $x_{h H}$ at the expense of $x_{l H}$. Doing so while keeping $x_{l H}\left[W_{l H}\left(q_{l H}\right)-W_{h L}\left(q_{l H}\right)\right]=$ $x_{h H}\left[W_{l H}\left(q_{h H}\right)-W_{h L}\left(q_{h H}\right)\right]$ requires an adjustment in $\lambda_{2}$, leading to $V W_{l H}$ decreasing and $V W_{h H}$ increasing. This process continues until we reach $\lambda_{2}^{* *}$ which corresponds to $V W_{l H}=$ $V W_{h H}$ (as defined in (28)). Since $\Delta \theta_{1}-\Delta \theta_{2} q_{h H}^{*}<0$, the corresponding qualities and $x$ 's are such that $q_{l H}^{*}<q_{l H}<q_{h H}<q_{h H}^{*}$ and $x_{h H}<x_{l H}$.

We now need to distinguish two cases depending whether $V W_{h L}>V W_{l H}=V W_{h H}$ or $V W_{l H}=V W_{h H}>V W_{h L}$.

(a) $V W_{h L}>V W_{l H}=V W_{h H}$ : Then we have reached the solution: $x_{l L}=x_{l L}^{F B}, x_{h L}=x_{h L}^{F B}$ and $x_{l H}^{F B}>x_{l H}>x_{h H}>x_{h H}^{F B}, q_{l L}=q_{h L}=\underline{q}$ and $q_{l H}^{*}<q_{l H}<q_{h H}<q_{h H}^{*}$ as defined by (28). This corresponds to Solution 2.1.c.

(b) $V W_{l H}=V W_{h H}>V W_{h L}$ : the buyer further increases his expected utility by increases $x_{l H}$ and $x_{h H}$ at the expense of $x_{h L}$ while keeping $V W_{h H}=V W_{l H}$ (that is keeping $\lambda_{2}$ and the $q$ 's fixed) and $U_{h L, l H}=U_{h L, h H}$ (thus $x_{h H}<x_{l H}$ ). This process does not affect the virtual welfares until $x_{h L}=x_{l H}$ and $\mathrm{IC}_{l L, l H}$ starts binding. We are now in a situation where $\mathrm{IC}_{l H, h H}, \mathrm{IC}_{l L, l H}, \mathrm{IC}_{l L, h L}, \mathrm{IC}_{h L, l H}$ and $\mathrm{IC}_{h L, h H}$ are all binding and $V W_{h L}<V W_{h H}=V W_{l H}$. From then on, the virtual welfares are those defined in (24) - (26) (note that $\lambda_{2}=\alpha_{h L}+\lambda_{5}-\lambda_{3}$ ). Let $\lambda_{5}^{*}$ such that $V W_{l H}=V W_{h L}$. Since there is no change in $\lambda_{3}$, the $q$ 's are not affected $\left(q_{l H}<q_{h H}\right)$ and the $x$ 's implicitly defined by $x_{l H}=x_{h L}$ and $U_{h L, h H}=U_{h L, l H}$ are not affected either. Thus we are exactly in the same 
situation as in 2(b) above, and the proof thus proceeds along the same lines. [Solution 2.1.d or 2.1.e] 